\title{
Technical Approach for the Development of DOE Building America Builders Challenge Technology Information Packages
}

D.R. Roberts and R. Anderson

Technical Report NREL/TP-550-44687

Revised August 2009 Anderson

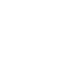




\section{Technical Approach for the Development of DOE Building America Builders Challenge Technology Information Packages}

D.R. Roberts and R. Anderson

Prepared under Task No. BET88001

Technical Report NREL/TP-550-44687

Revised August 2009

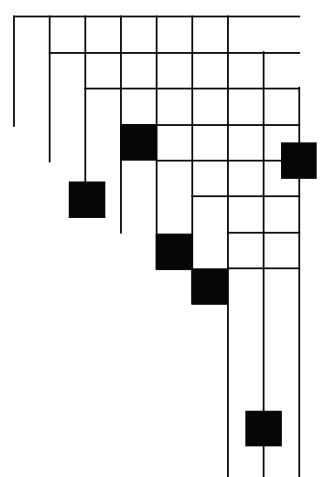

The addition of Appendix $D$ is the only revision to the January 2009 version of this report. 


\section{NOTICE}

This report was prepared as an account of work sponsored by an agency of the United States government. Neither the United States government nor any agency thereof, nor any of their employees, makes any warranty, express or implied, or assumes any legal liability or responsibility for the accuracy, completeness, or usefulness of any information, apparatus, product, or process disclosed, or represents that its use would not infringe privately owned rights. Reference herein to any specific commercial product, process, or service by trade name, trademark, manufacturer, or otherwise does not necessarily constitute or imply its endorsement, recommendation, or favoring by the United States government or any agency thereof. The views and opinions of authors expressed herein do not necessarily state or reflect those of the United States government or any agency thereof.

Available electronically at http://www.osti.gov/bridge

Available for a processing fee to U.S. Department of Energy and its contractors, in paper, from:

U.S. Department of Energy

Office of Scientific and Technical Information

P.O. Box 62

Oak Ridge, TN 37831-0062

phone: 865.576 .8401

fax: 865.576 .5728

email: mailto:reports@adonis.osti.gov

Available for sale to the public, in paper, from:

U.S. Department of Commerce

National Technical Information Service

5285 Port Royal Road

Springfield, VA 22161

phone: 800.553.6847

fax: 703.605.6900

email: orders@ntis.fedworld.gov

online ordering: http://www.ntis.gov/ordering.htm 


\section{Acknowledgments}

The authors wish to thank Ron Judkoff and Dane Christensen of NREL for providing technical review and comments, and Stefanie Woodward of NREL for providing editorial review and revision. 


\section{Executive Summary}

The U.S. Department of Energy (DOE) has issued a challenge to the homebuilding industry to build 220,000 high-performance homes by 2012. The initiative is called Builders Challenge. To qualify, homes must meet the requirements of one of three compliance paths established by DOE: performance path, prescriptive path, or participating in a partner program. The performance path, expected to be the most widely used, requires that a home receive a home energy rating and score 70 or better (lower) on the EnergySmart Home Scale.

To provide builders with specific design specifications and a prescriptive path to compliance, DOE is developing a series of Builders Challenge Technology Information Packages (BC-TIPs) - climate-specific lists of energy features that must be installed in a home to meet minimum program requirements.

The National Renewable Energy Laboratory used the BEopt, REM/Rate, and EnergyGauge software programs to develop an initial batch of five BC-TIPs. The goal was to achieve optimal cost-effective approaches to meeting the 70 EnergySmart Home Scale requirement. Additional premium-efficiency packages were developed for each of the initial five climates to demonstrate higher levels of cost-effective energy savings. BCTIP marketing materials targeting builders and consumers have been developed for the initial set of five climates and are available on the Building America Web site.

This report describes the technical approach used to develop the BC-TIPs.
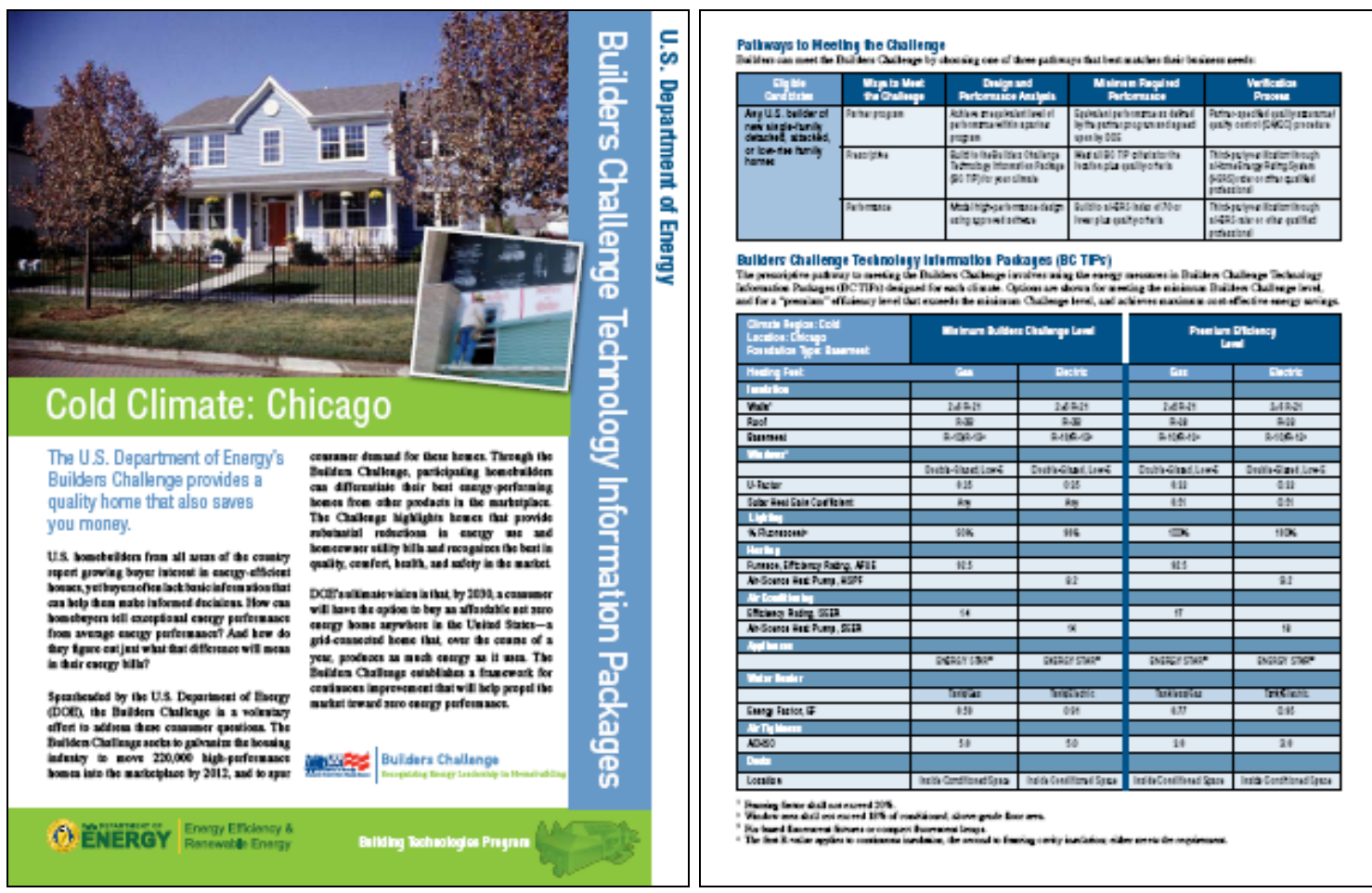


\section{Contents}

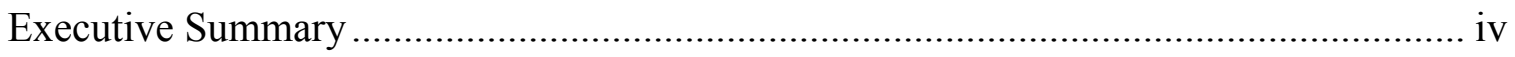

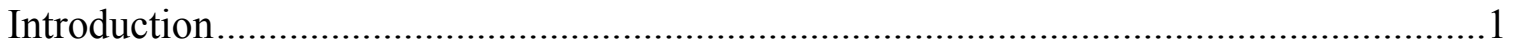

Initial Set of Technical Information Packages .............................................................

Technical Approach for the Development of Builders Challenge Technical Information

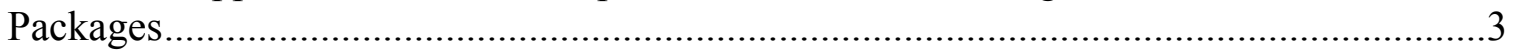

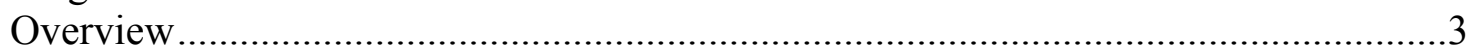

Prototype Building Definition............................................................................. 4

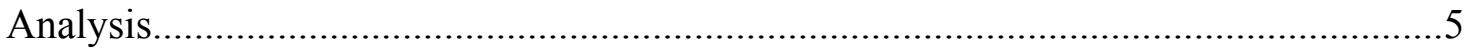

Finalizing Technology Information Packages .........................................................

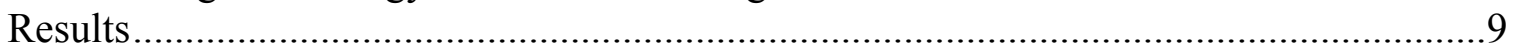

Technical Information Package Design Limitations......................................................11

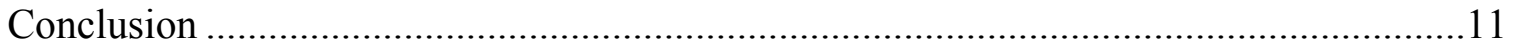

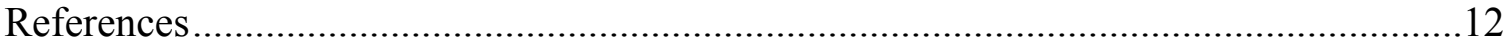

Appendix A: Example BEopt Input Echo Report - Phoenix, Gas Heating ................... A-1

Appendix B: Example REM/Rate Building File Report - Phoenix, Gas Heating...........B-1

Appendix C: Example EnergyGauge Building Input Report - Phoenix, Gas Heating ...C-1

Appendix D: BEopt Results (added August 2009) ................................................... D-1 


\section{Figures}

Figure 1. Builders Challenge EnergySmart Home Scale................................................1

Figure 2. Example Builders Challenge Technology Information Package.........................3

Figure 3. SketchUp rendering of prototype model ...................................................4

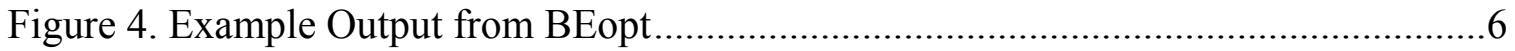

Figure 5. BEopt economic factors and energy source multipliers used in TIP analysis......7

Figure 6. Analysis flow for developing BC-TIPs ......................................................

Figure 7. Sample package table from BC-TIP document ...........................................10

Figure 8. Example energy savings and cost of upgrades from BC-TIP document............10

\section{Tables}

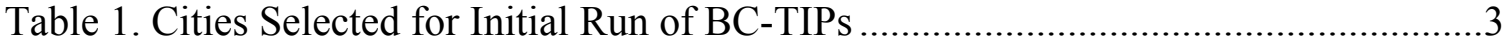

Table 2. Foundation Types Used in the Prototype Models............................................5

Table 3. Prototype Model Characteristics........................................................................ 5

Table 4. BEopt Default Statewide Average Fuel Costs Used to Develop TIPs .................6

Table 5. E-Scale Indices for Prototype Homes ............................................................. 


\section{Introduction}

The U.S. Department of Energy (DOE) has issued a challenge to the homebuilding industry to build 220,000 high-performance homes by 2012. The initiative is called Builders Challenge. To qualify, homes must meet the requirements of one of three compliance paths established by DOE: performance path, prescriptive path, or participating in a partner program. In addition to the requirements unique to each path, a common set of quality criteria must be verified via third-party inspection.

The performance path, expected to be the most widely used, requires that a home achieve a 70 or better (lower) on the EnergySmart Home Scale (E-Scale). The E-Scale (Figure 1) allows homebuyers to understand at a glance how the performance of a particular home compares to that of others. This scale is the same as that used in the home energy rating system (HERS) industry as defined by the industry organization, Residential Energy Services Network (RESNET). ${ }^{1}$ A home that scores 70 on the E-Scale will use approximately $70 \%$ of the energy as the same home built to meet the minimum requirements of the 2004 International Energy Conservation Code (IECC).

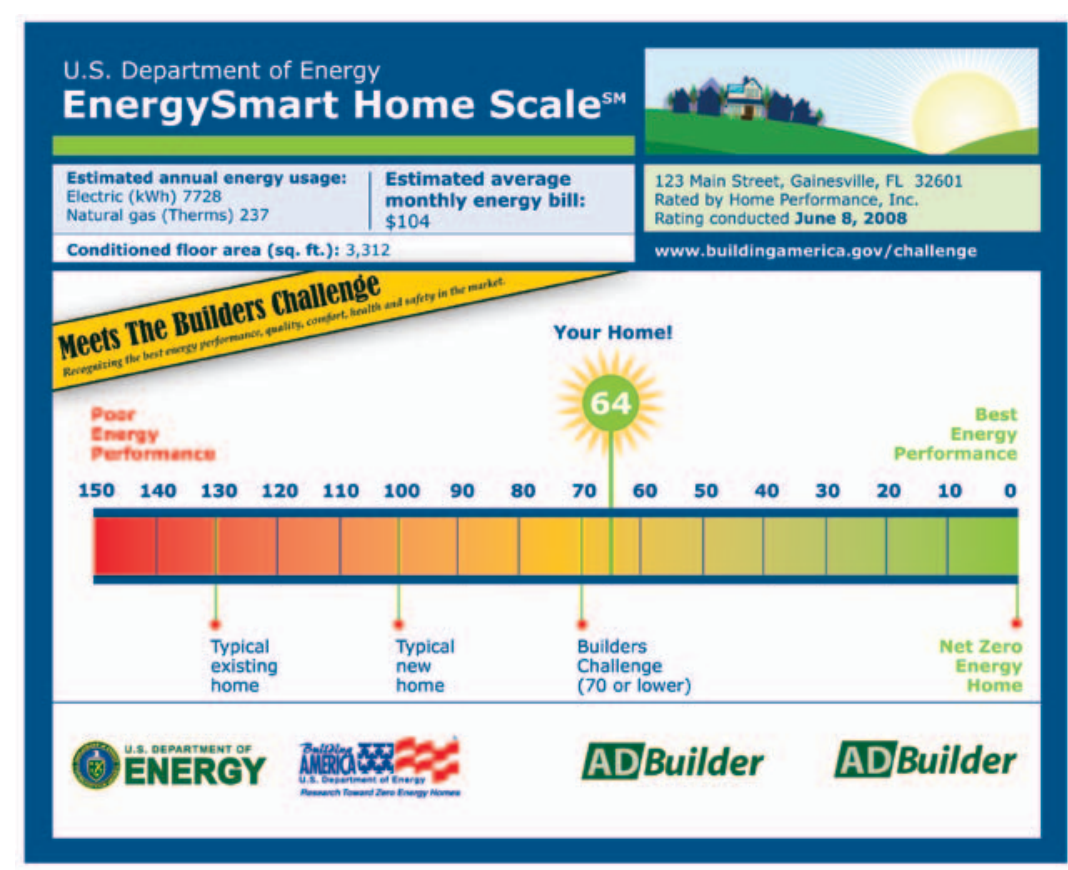

Figure 1. Builders Challenge EnergySmart Home Scale

The E-Scale Index is calculated using computer software programs that compare the estimated energy use of a proposed home design to the energy use of the same home built to meet the minimum requirements of the 2004 IECC. The software user, generally a HERS rater, will modify features of the home to achieve the target E-Scale Index, in this case 70 or lower. This is commonly referred to as the performance path, because any

\footnotetext{
${ }^{1} 2006$ Mortgage Industry National Home Energy Rating System Standards, Residential Energy Services Network, Inc., May 19, 2007.
} 
combination of design features can be used to meet the overall performance objective for the home.

To provide builders with examples of specific design specifications and make the Builders Challenge more accessible in markets without a strong HERS presence, DOE developed a prescriptive path to meeting the Challenge. To facilitate this path, the National Renewable Energy Laboratory (NREL) was tasked with creating Builders Challenge Technology Information Packages (BC-TIPs).

A BC-TIP provides a list of energy features that must be installed in the home to meet minimum program requirements (Figure 2). TIPs offer an alternative to the performancebased approach. Builders who do not have access to HERS raters in their market, or who prefer the simplicity of a prescriptive list, can implement the features listed in a TIP for their climate region.

TIPs do not offer the full flexibility provided by the performance path and, by definition, cannot provide full credit for the performance of specific building characteristics and components.

This document outlines NREL's technical approach in developing the BC-TIPs for Builders Challenge. 

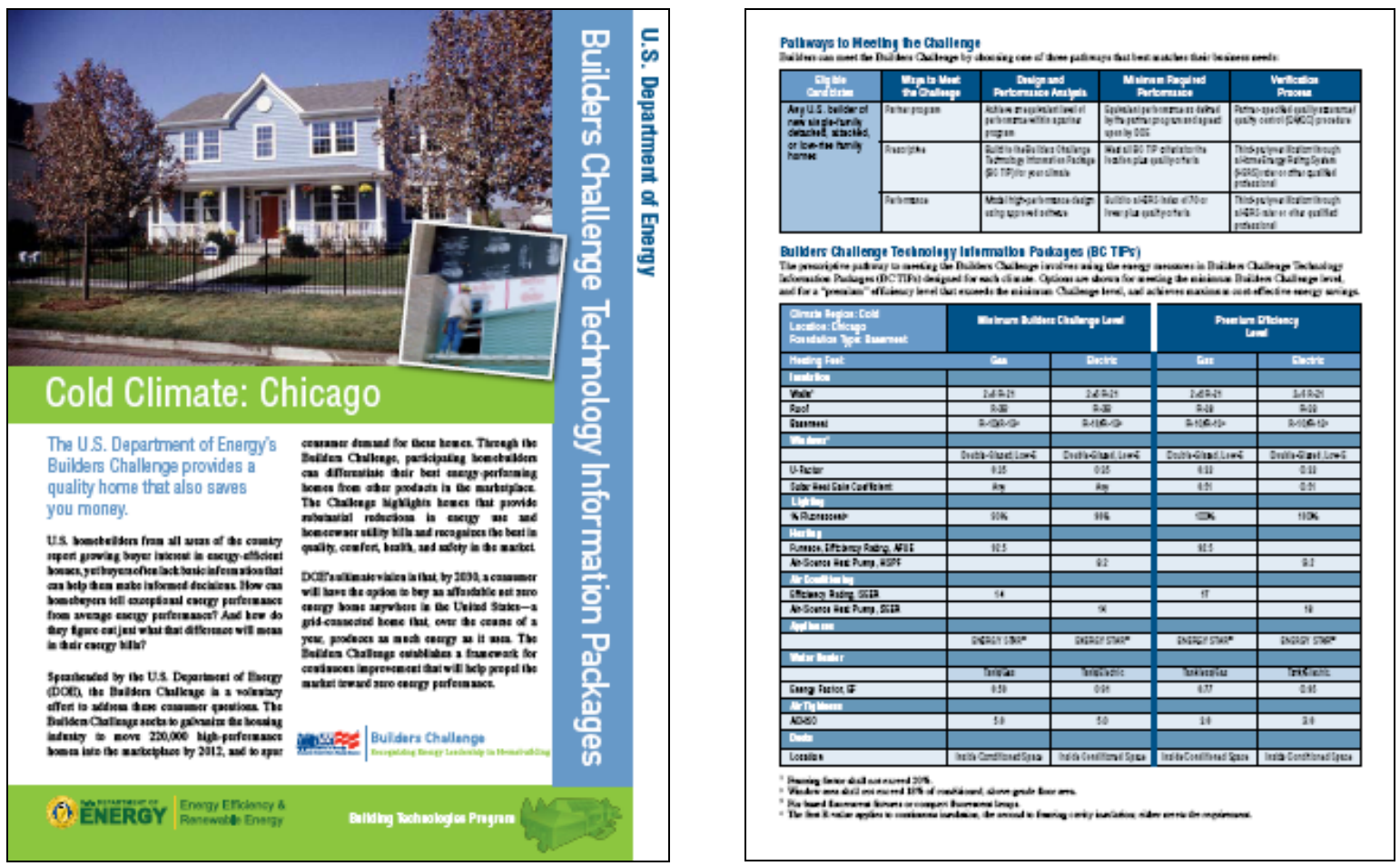

Figure 2. Example Builders Challenge Technology Information Package

\section{Initial Set of Technical Information Packages}

An initial set of BC-TIPs has been developed that includes one major metropolitan area in each of the five Building America Climate Regions: hot-humid, hot/mixed-dry, cold, mixed-humid, and marine. BC-TIPs were developed for all-electric and gas/electric homes. Table 1 shows the cities selected to initially represent each climate zone.

Table 1. Cities Selected for Initial Run of BC-TIPs

\begin{tabular}{|c|c|}
\hline Climate Zone & City \\
\hline Hot-Humid & Houston \\
\hline Hot/Mixed-Dry & Phoenix \\
\hline Cold & Chicago \\
\hline Mixed-Humid & Atlanta \\
\hline Marine & Seattle \\
\hline
\end{tabular}

\section{Technical Approach for the Development of Builders Challenge Technical Information Packages}

\section{Overview}

To develop the initial batch of BC-TIPs, NREL created energy models of prototypical, single-family homes for each of the five Building America Climate Regions. NREL used 
BEopt $^{2}$ energy design optimization software, REM/Rate ${ }^{3}$ HERS software, and EnergyGauge ${ }^{4}$ HERS software to develop and analyze the models. The BEopt software tool was developed at NREL to identify optimal building energy designs aimed at minimizing the total of the amortized cost of improvements and the cost of energy. REM/Rate and EnergyGauge are two widely used HERS software programs accredited by RESNET $^{5}$ that produce the E-Scale Index. BEopt, REM/Rate, and EnergyGauge all utilize energy simulation engines that have passed the HERS building energy simulation test (Judkoff and Neymark, 1995).

BEopt was used to identify least-cost approaches to meeting two performance goals, each presented in the TIP documents: (1) minimum requirements for the Builders Challenge, and (2) a Building America package designed to achieve maximum cost-effective energy savings. The REM/Rate and EnergyGauge software were used to ensure the Builders Challenge-level recommendations are consistent with performance-based approaches to reaching the 70 E-Scale threshold.

\section{Prototype Building Definition}

The prototype building used to develop the TIPs is a 2,500- $\mathrm{ft}^{2}, 2$-story, single-family home (Figure 3), sitting on a climate-appropriate foundation - slab, crawlspace, or conditioned basement as shown in Table 2. The home has a 1.3:1 aspect ratio, with the front (a long side) facing west. The home has total (frame and glass) window area equal to $18 \%$ of the conditioned floor area distributed equally on all four sides of the home. Detailed prototype model characteristics are shown in Table 3.

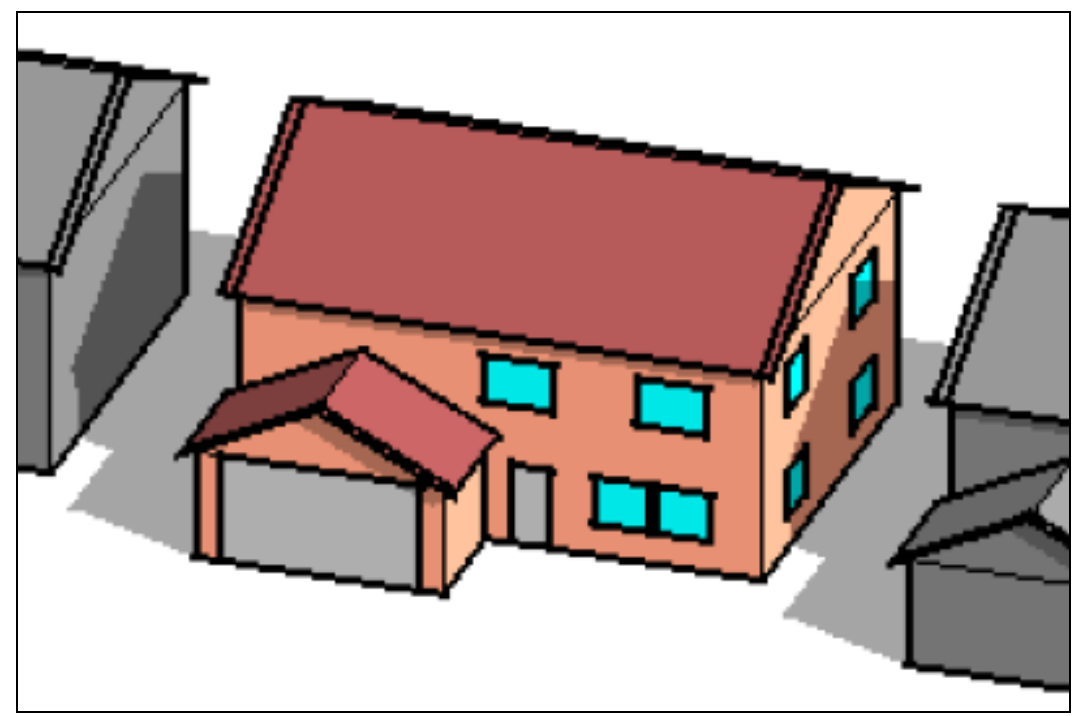

Figure 3. SketchUp rendering of prototype model

\footnotetext{
2 v0.8.7, National Renewable Energy Laboratory

${ }^{3}$ v12.6, Architectural Energy Corporation

${ }^{4}$ v2.8.00, Florida Solar Energy Center

${ }^{5}$ National Registry of Accredited Rating Software Programs, Residential Energy Services Network, Inc.
} 
Table 2. Foundation Types Used in the Prototype Models

\begin{tabular}{|c|c|}
\hline City & Foundation Type \\
\hline Houston & Slab \\
\hline Phoenix & Slab \\
\hline Chicago & Conditioned Basement \\
\hline Atlanta & Crawlspace \\
\hline Seattle & Crawlspace \\
\hline
\end{tabular}

Table 3. Prototype Model Characteristics

\begin{tabular}{|l|l|}
\hline Conditioned floor area & $2,500 \mathrm{ft}^{2}, 3,750 \mathrm{ft}^{2} \mathrm{w} / \mathrm{basement}$ \\
\hline Conditioned volume & $22,500 \mathrm{ft}^{3}, 33,750 \mathrm{ft}^{3} \mathrm{w} / \mathrm{b}$ basement \\
\hline Number of stories & 2 \\
\hline Number of bedrooms & 3 \\
\hline Gross above-grade wall area & $2,574 \mathrm{ft}^{2}$ \\
\hline Window area & $450 \mathrm{ft}^{2}, 500 \mathrm{ft}^{2}$ w/basement \\
\hline Window orientation & Equally distributed, 4 cardinal directions \\
\hline Door area & $40 \mathrm{ft}^{2}$ \\
\hline Setpoint temperatures & 68 heating, 78 cooling \\
\hline Mechanical ventilation & Exhaust only, ASHRAE 62.2 levels \\
\hline Internal gains & Building America Benchmark (Hendron, 2008) \\
\hline Lighting/appliance/plug schedules & Building America Benchmark \\
\hline
\end{tabular}

In developing the prototype building design, small studies were undertaken to examine the sensitivity of the E-Scale Index to window orientation and house size. Both studies involved changing HERS software prototype models to represent extreme cases:

- Window orientation was changed from equally distributed to $50 \%$ west-facing, $25 \%$ east-facing, and $12.5 \%$ north- and south-facing. This change increased the EScale Index for the 10 prototype models (five climates, gas \& electric space heating) an average of 0.78 points.

- House size was reduced by removing the second floor from the prototype, resulting in 1,250- $\mathrm{ft}^{2}$ homes without the basement and 2,500- $\mathrm{ft}^{2}$ homes with the conditioned basement. This change increased the E-Scale Index by an average of 6.3 points for the 10 prototype models.

\section{Analysis}

BEopt was used in conjunction with the prototype building definition to develop the BCTIPs. It produces building designs that minimize combined construction and energy costs by using the DOE-2.2 and TRNSYS energy simulation programs to automate a sequential search technique for locating least-cost solutions on a path toward net zero energy. The software and underlying methodology are described in detail by Christensen et al. (2005, 2006) and Horowitz et al. (2008).

Figure 4 shows typical output from BEopt. Each point of the graph indicates a unique combination of energy design features. The dark line at the bottom of the points indicates 
the least-cost solution to achieving the source energy savings indicated on the $X$ axis. An approximation of the Builders Challenge target is indicated on the graph. Although the actual Builders Challenge threshold is 70 or better on the E-Scale, source energy savings relative to the 2006 IECC were typically $18 \%-25 \%$ for the climates and heating fuels analyzed. Building configurations in this area were examined and analyzed as potential Builders Challenge-level packages. Additionally, premium-efficiency packages were selected from points at or near the minimum cost point. An example BEopt input file is included in Appendix A.

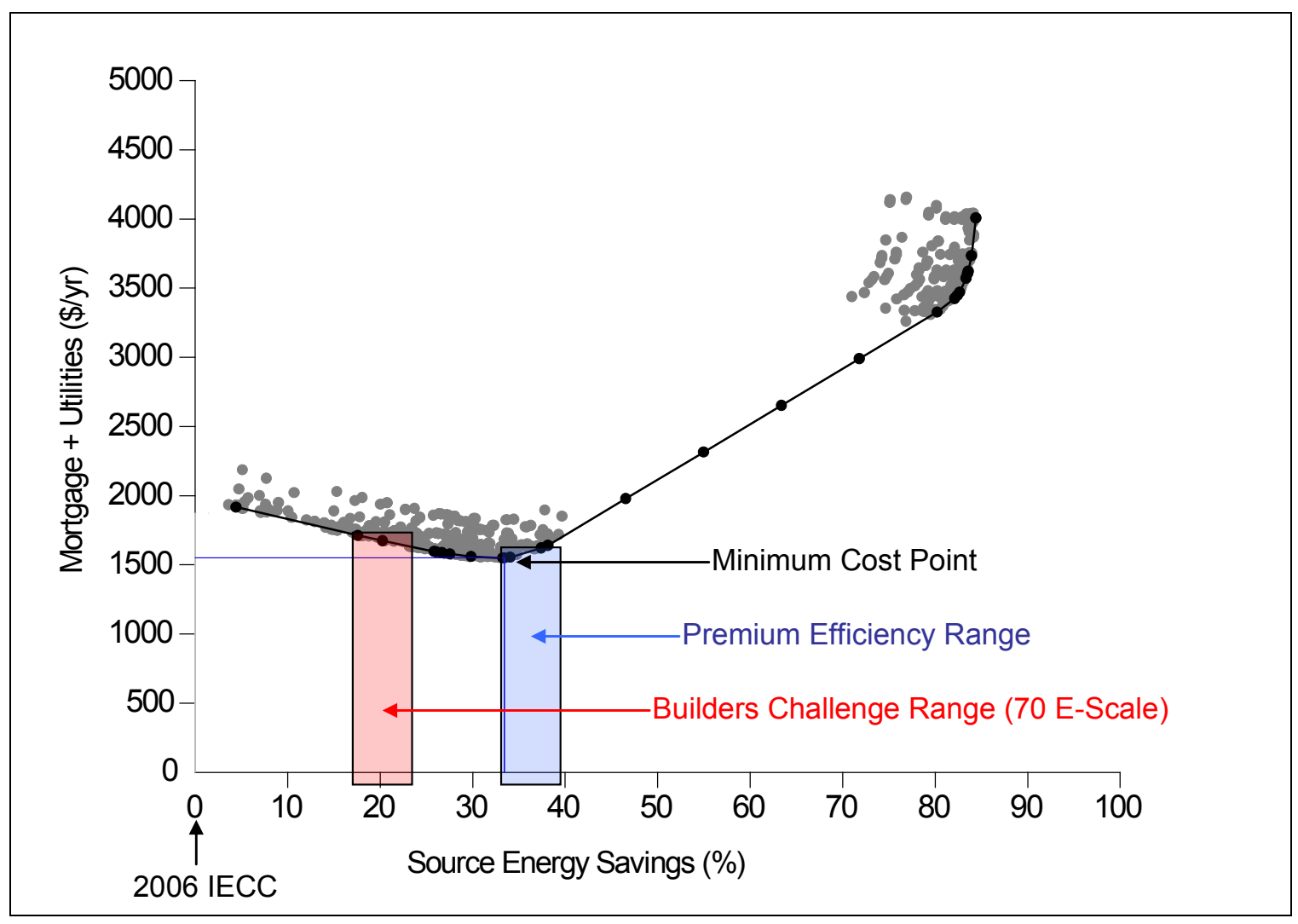

Figure 4. Example Output from BEopt

BEopt analysis was performed for each climate listed in Table 1. Standard BEopt default values were used in the analysis: statewide average utility rates (Table 4), economic factors, and source energy multipliers (Figure 5).

Table 4. BEopt Default (EIA 2006) Statewide Average Fuel Costs Used to Develop TIPs

\begin{tabular}{|c|c|c|}
\hline City & $\mathbf{\$} / \mathbf{k W h}$ & $\mathbf{\$} /$ Therm \\
\hline Houston & 0.1142 & 1.1544 \\
\hline Phoenix & 0.0763 & 1.4376 \\
\hline Chicago & 0.0685 & 1.0754 \\
\hline Atlanta & 0.0792 & 1.7789 \\
\hline Seattle & 0.0608 & 1.2786 \\
\hline
\end{tabular}




\begin{tabular}{|c|c|c|}
\hline \multicolumn{3}{|l|}{ Mortgage } \\
\hline Mortgage Period & 30 & years \\
\hline Mortgage Interest Rate (Nominal) & 7.0 & $\%$ \\
\hline Marginal Income Tax Rate & 28.0 & $\%$ \\
\hline \multicolumn{3}{|l|}{ Economics- } \\
\hline Project Analysis Period & 30 & years \\
\hline Inflation Rate & 3.0 & $\%$ \\
\hline Discount Rate (Nominal) & 5.0 & $\%$ \\
\hline \multicolumn{3}{|l|}{$\lceil$ Multipliers } \\
\hline Electric Source/Site Ratio & 3.365 & \\
\hline Gas Source/Site Ratio & 1.092 & \\
\hline Electric Carbon Factor & 1.670 & $\mathrm{lb} / \mathrm{kWh}$ \\
\hline Gas Carbon Factor & 14.740 & Ib/therr \\
\hline Efficiency Cost Multiplier & 1.0 & \\
\hline
\end{tabular}

Figure 5. BEopt economic factors and energy source multipliers used in TIP analysis

To the extent possible, the 2006 IECC Standard Reference was used for the BEopt analysis so the resulting energy cost savings would be relative to current code. This reference is not built in to BEopt, and was hand-configured in the software. There are two aspects of the IECC Standard Reference as described in Section 404 of the 2006 IECC that could not be precisely configured: Distributions System Efficiency value of 0.8 , and window U-value and SHGC. In these cases the closest possible approximations were used - code-level ducts and windows that most closely matched the code requirements. This reference is the point from which the cost of energy efficiency improvements and energy cost savings reported in the BC-TIPs are calculated. Because these results are not critical - reflecting typical, not actual performance - a close approximation of this "current code" suffices in the analysis.

Potential Builders Challenge-level prescriptive packages identified using BEopt were further analyzed using RESNET-accredited HERS software. It is important that the BCTIPs be checked to ensure they achieve an E-Scale Index of 70 or lower (analogous to a 70 on the HERS Scale).

Analogous prototype models were created in the REM/Rate and EnergyGauge HERS software programs. The HERS software prototype models were configured to reflect energy efficiency features from BEopt associated with a specific point on the BEopt minimum-cost curve. If the E-Scale Index from the software was higher than 70, a point further along the savings curve was selected from BEopt, and the HERS prototypes reconfigured and reevaluated. Figure 6 shows the iterative process that was followed until a least-cost solution resulting in a HERS (E-Scale) Index below 70 was identified for each prototype home. 


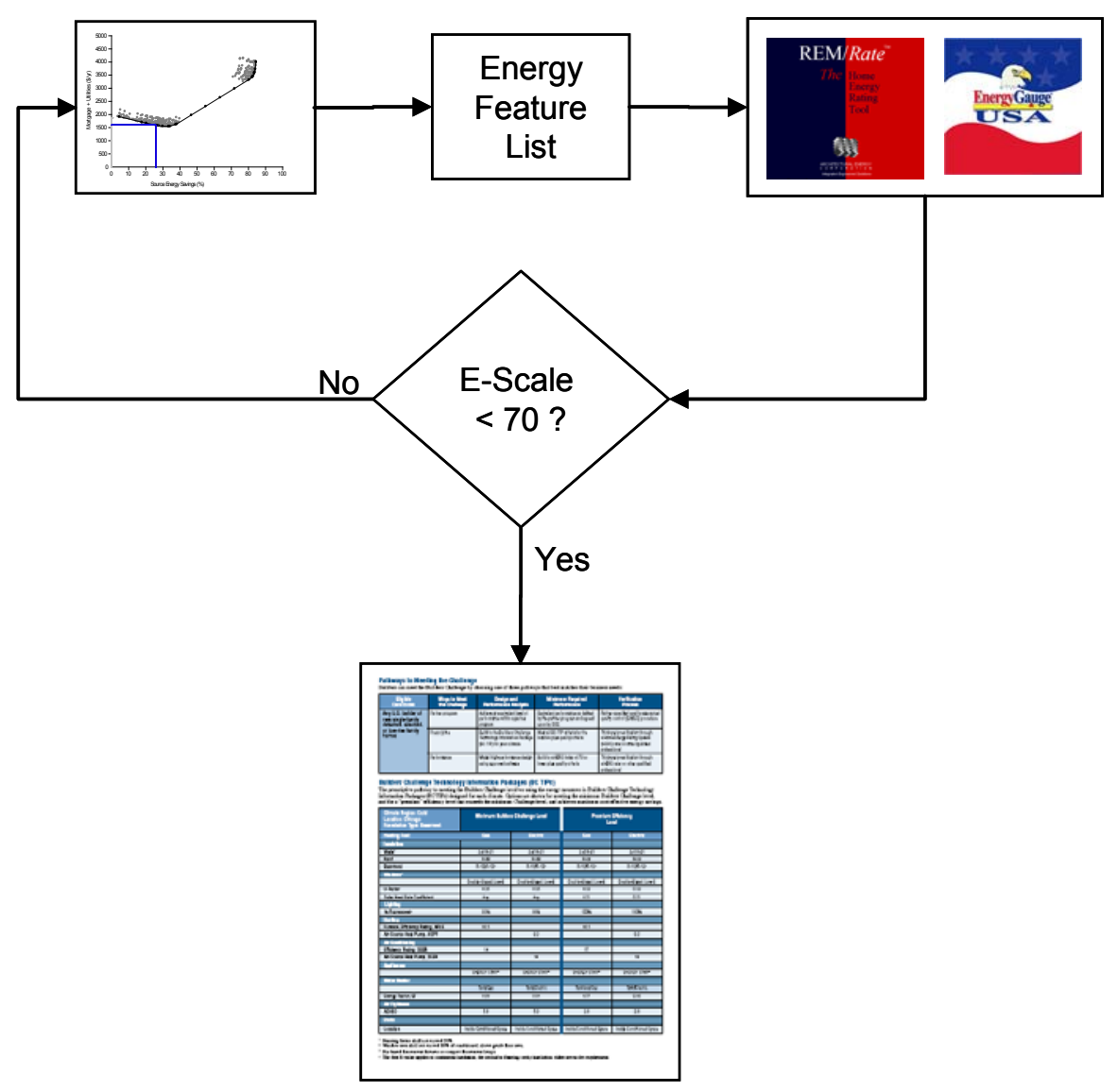

Figure 6. Analysis flow for developing BC-TIPs

The data presented in Figure 4 demonstrate that there are many solutions near the minimum cost point on the curve, and that source energy savings as high as $35 \%$ relative to 2006 IECC can be reached without deviating significantly from minimum cost. The cost for the $35 \%$ savings point is far below the annual cost point for a 2006 IECCcompliant home, which is the baseline for the data shown in Figure 4. To highlight the benefit of efficiency levels beyond the E-Scale criterion of 70, a second, higher performance package is included in the TIP documents. These packages generally produce greater cost savings for the homeowner, and in all cases produce greater source energy savings with positive cash flow.

\section{Finalizing Technology Information Packages}

Once potential Builders Challenge-level packages were identified for each climate and fuel type, they were vetted with the Builders Challenge Technical Working Group and adjusted slightly for consistency, flexibility, and practicality. For example, the Group requested $100 \%$ fluorescent lighting be reduced to $90 \%$ to provide some flexibility. The Group also decided that minimum seasonal energy efficiency ratio (SEER) values for air conditioners and heat pumps should be 14.0, that the minimum furnace efficiency should be 82.0 annual fuel utilization efficiency (AFUE), and that required window solar heat 
gain coefficient (SHGC) values should be no lower than $0.35^{6}$ to accommodate product availability. These changes were made to the prototype models in the HERS software, and the HERS indices were reevaluated.

\section{Results}

Table 5 shows the E-Scale Indices for the Builders Challenge performance level for each prototype model developed for this analysis.

Table 5. E-Scale Indices for Prototype Homes at Builders Challenge Performance Level

\begin{tabular}{|c|c|c|}
\hline City/Heating Fuel & REM/Rate & EnergyGauge \\
\hline Houston/Gas & 68 & 64 \\
\hline Houston/Electric & 68 & 65 \\
\hline Phoenix/Gas & 67 & 63 \\
\hline Phoenix/Electric & 67 & 64 \\
\hline Chicago/Gas & 62 & 64 \\
\hline Chicago/Electric & 68 & 69 \\
\hline Atlanta/Gas & 66 & 63 \\
\hline Atlanta/Electric & 68 & 65 \\
\hline Seattle/Gas & 66 & 69 \\
\hline Seattle/Electric & 67 & 70 \\
\hline
\end{tabular}

Figure 7 shows a typical BC-TIP energy package, clipped from the builder-targeted marketing flyer. In addition to the energy packages, the estimated costs and savings associated with each package are also listed in the BC-TIP marketing flyer (Figure 8). As discussed earlier, these savings and construction costs are calculated by BEopt, relative to the hand-configured 2006 IECC Standard Reference. The complete TIP document and other BC-TIPs are available on the Building America Web site.

\footnotetext{
${ }^{6}$ The 2009 IECC will require SHGC values lower than 0.35 in warm climates, and the 2010 ENERGY $\mathrm{STAR}^{\circledR}$ window specification is expected to be even more stringent.
} 


\begin{tabular}{|c|c|c|c|c|}
\hline $\begin{array}{l}\text { Climate Region: Cold } \\
\text { Location: Chicago } \\
\text { Foundation Type: Basement }\end{array}$ & \multicolumn{2}{|c|}{ Minimum Builders Challenge Level } & \multicolumn{2}{|c|}{$\begin{array}{c}\text { Premium Efficiency } \\
\text { Level }\end{array}$} \\
\hline Heating Fuel: & Gas & Electric & Gas & Electric \\
\hline \multicolumn{5}{|l|}{ Insulation } \\
\hline Walls $^{1}$ & $2 \times 6 \mathrm{R}-21$ & $2 \times 6 \mathrm{R}-21$ & $2 \times 6 \mathrm{R}-21$ & $2 \times 6 \mathrm{R}-21$ \\
\hline Roof & $\mathrm{R}-38$ & $\mathrm{R}-38$ & $\mathrm{R}-38$ & $\mathrm{R}-38$ \\
\hline Basement & $\mathrm{R}-10 / \mathrm{R}-13^{4}$ & $R-10 / R-13^{4}$ & $R-10 / R-13^{4}$ & $R-10 / R-13^{4}$ \\
\hline \multicolumn{5}{|l|}{ Windows $^{2}$} \\
\hline & Double-Glazed, Low-E & Double-Glazed, Low-E & Double-Glazed, Low-E & Double-Glazed, Low-E \\
\hline U-Factor & 0.35 & 0.35 & 0.33 & 0.33 \\
\hline Solar Heat Gain Coefficient & Any & Any & 0.51 & 0.51 \\
\hline \multicolumn{5}{|l|}{ Lighting } \\
\hline$\%$ Fluorescent Fixtures $^{3}$ & $90 \%$ & $90 \%$ & $100 \%$ & $100 \%$ \\
\hline \multicolumn{5}{|l|}{ Heating } \\
\hline Furnace, Efficiency Rating, AFUE & 92.5 & & 92.5 & \\
\hline Air-Source Heat Pump, HSPF & & 8.2 & & 9.2 \\
\hline \multicolumn{5}{|l|}{ Air Conditioning } \\
\hline Efficiency Rating, SEER & 14 & & 17 & \\
\hline Air-Source Heat Pump, SEER & & 14 & & 18 \\
\hline \multicolumn{5}{|l|}{ (Builder-Supplied) Appliances } \\
\hline & ENERGY STAR $^{\circ}$ & ENERGY STAR $^{\circ}$ & ENERGY STAR $^{\circ}$ & ENERGY STAR $^{\circ}$ \\
\hline \multicolumn{5}{|l|}{ Water Heater } \\
\hline & Tank/Gas & Tank/Electric & Tankless/Gas & Tank/Electric \\
\hline Energy Factor, EF & 0.59 & 0.91 & 0.77 & 0.95 \\
\hline \multicolumn{5}{|l|}{ Air Tightness } \\
\hline $\mathrm{ACH} 50$ & 5.0 & 5.0 & 3.0 & 3.0 \\
\hline \multicolumn{5}{|l|}{ Ducts } \\
\hline Location & $\begin{array}{l}\text { Inside Conditioned } \\
\text { Space }\end{array}$ & $\begin{array}{l}\text { Inside Conditioned } \\
\text { Space }\end{array}$ & $\begin{array}{l}\text { Inside Conditioned } \\
\text { Space }\end{array}$ & $\begin{array}{l}\text { Inside Conditioned } \\
\text { Space }\end{array}$ \\
\hline \multicolumn{5}{|c|}{$\begin{array}{l}\text { Framing factor shall not exceed } 20 \% \text {. } \\
2 \text { Window area shall not exceed } 18 \% \text { of conditioned, above-grade floor area. } \\
{ }^{3} \text { Pin-based fluorescent fixtures or compact fluorescent lamps. } \\
4 \text { The first R-value applies to continuous insulation, the second to framing cavity insulation; either meets the requirement. }\end{array}$} \\
\hline
\end{tabular}

Figure 7. Sample package table from BC-TIP document

\begin{tabular}{|c|c|c|c|c|}
\hline \multirow{2}{*}{$\begin{array}{l}\text { Upgraded Energy } \\
\text { Savings Levels }\end{array}$} & \multicolumn{2}{|c|}{$\begin{array}{l}\text { Minimum Builders } \\
\text { Challenge Level }\end{array}$} & \multicolumn{2}{|c|}{$\begin{array}{c}\text { Premium Efficiency } \\
\text { Level }\end{array}$} \\
\hline & Gas & Electric & Gas & Electric \\
\hline Savings on annual utility bill ${ }^{1}$ & $\$ 375$ & $\$ 366$ & $\$ 570$ & $\$ 458$ \\
\hline $\begin{array}{l}\text { Increase in annual mortgage payment } \\
\text { from energy upgrades }{ }^{2}\end{array}$ & $\$ 178$ & $\$ 151$ & $\$ 254$ & $\$ 204$ \\
\hline Net annual savings & $\$ 197$ & $\$ 215$ & $\$ 316$ & $\$ 254$ \\
\hline
\end{tabular}

Figure 8. Example energy savings and cost of upgrades from BC-TIP document 


\section{Technical Information Package Design Limitations}

Because of the TIP prototype building definition, builders who use TIPs will not be able to take advantage of performance tradeoffs based on window area, window location, building orientation, reduced infiltration, etc. Builders interested in receiving full credit for specific building designs and performance features are encouraged to use the performance path. A home that has a window area greater than $18 \%$ of conditioned floor area is required to use the performance path.

\section{Conclusion}

NREL used the BEopt, REM/Rate, and EnergyGauge software programs to develop an initial batch of five TIPs for DOE's Builder Challenge. The goal was to achieve optimal cost-effective approaches to meeting the 70 E-Scale requirement of the Challenge.

Premium-efficiency packages were also developed for each of the initial five climates to demonstrate higher levels of cost-effective energy savings.

TIPs quickly and effectively communicate the technical specifications required to meet the Builders Challenge. TIPs do not provide the full design flexibility of the performance path.

BC-TIP marketing materials targeting builders and consumers have been developed for the initial set of five climates and are available on the Building America Web site. 


\section{References}

Christensen, C.; Horowitz, S.; Givler, T.; Courtney, A.; Barker, G. (2005). BEopt: Software for Identifying Optimal Building Designs on the Path to Zero Net Energy. NREL/CP-550-37733. Golden, CO: National Renewable Energy Laboratory.

Christensen, C.; Anderson, R.; Horowitz, S.; Courtney, A.; Spencer, J. (2006). BEopt(TM) Software for Building Energy Optimization: Features and Capabilities. NREL/TP-550-39929. Golden, CO: National Renewable Energy Laboratory.

Hendron, R. (2008). Building America Research Benchmark Definition: Updated December 20, 2007. NREL Report No. TP-550-42662. Golden, CO: National Renewable Energy Laboratory.

Horowitz, S.; Christensen, C.; Brandemuehl, M.; Krarti, M. (2008). Enhanced Sequential Search Methodology for Identifying Cost-Optimal Building Pathways. NREL/CP-55043238. Golden, CO: National Renewable Energy Laboratory.

Judkoff, R.; Neymark, J. (1995). Home Energy Rating System Building Energy Simulation Test (HERS BESTEST). NREL/TP-472-7332a. Golden, CO: National Renewable Energy Laboratory.

International Code Council, Inc. (2006). 2006 International Energy Conservation Code. ISBN-13: 978-1-58001-270-6. 
Appendix A: Example BEopt Input Echo Report - Phoenix, Gas Heating 


\begin{tabular}{|c|c|c|c|}
\hline GROUP & INPUT VARIABLE & TVALUE & UNITS \\
\hline Location & USA_AZ_Phoenix_TMY2 & & \\
\hline \multirow[t]{10}{*}{ Geometry } & Total Finished Floor Area (Above Grade) & 2500 & $\mathrm{ft}^{2}$ \\
\hline & \# Floors (Above Grade) & 2 & \\
\hline & Ceiling Height & 9 & $\mathrm{ft}$ \\
\hline & Garage & Two Car & \\
\hline & Garage Protrusion: & 50 & $\%$ \\
\hline & Garage Position: & Left & \\
\hline & Roof & Gable & \\
\hline & Roof Pitch: & 0.5 & \\
\hline & Number of Bedrooms & 3 & \\
\hline & Number of Bathrooms & 2 & \\
\hline \multirow[t]{18}{*}{ Economics } & Elec. Marginal & 0.0763 & $\$ / \mathrm{kWh}$ \\
\hline & Elec. Fixed & 8 & \$/month \\
\hline & Elec. Average & 0.0815 & $\$ / \mathrm{kWh}$ \\
\hline & Elec. Excess Sellback Rate & 0.0763 & $\$ / \mathrm{kWh}$ \\
\hline & Gas Marginal & 1.4376 & $\$$ /therm \\
\hline & Gas Fixed & 8 & $\$ /$ month \\
\hline & Gas Average & 1.684 & $\$$ /therm \\
\hline & Mortgage Period & 30 & years \\
\hline & Mortgage Interest Rate (Nominal) & 7 & $\%$ \\
\hline & Marginal Income Tax Rate & 28 & $\%$ \\
\hline & Project Analysis Period & 30 & years \\
\hline & Inflation Rate & 3 & $\%$ \\
\hline & Discount Rate (Nominal) & 5 & $\%$ \\
\hline & Electric Source/Site Ratio & 3.365 & \\
\hline & Gas Source/Site Ratio & 1.092 & \\
\hline & Gas Carbon Factor & 14.74 & \\
\hline & Electric Carbon Factor & 1.67 & \\
\hline & Efficiency Cost Multiplier & 1 & \\
\hline \multirow[t]{3}{*}{ Photovoltaics } & PV Module & Generic & \\
\hline & Installed Cost & 7.5 & $\$ / W D C$ \\
\hline & Derate Factor & 15 & $\%$ \\
\hline
\end{tabular}




\begin{tabular}{|c|c|c|c|c|c|c|c|}
\hline GROUP & CATEGORY & REF & SELECTED & OPTION NAME & UNIT COST & LIFETIME & \\
\hline \multicolumn{8}{|l|}{ Building } \\
\hline & Orientation & & & & & & \\
\hline & & & & North-facing & $\$ 0.00$ & 30 & years \\
\hline & & $x$ & $x$ & West-facing & $\$ 0.00$ & 30 & years \\
\hline & & & & South-facing & $\$ 0.00$ & 30 & years \\
\hline & & & & East-facing & $\$ 0.00$ & 30 & years \\
\hline & & & & Southeast & $\$ 0.00$ & 30 & years \\
\hline & & & & Southwest & $\$ 0.00$ & 30 & years \\
\hline & & & & Northeast & $\$ 0.00$ & 30 & years \\
\hline & & & & Northwest & $\$ 0.00$ & 30 & years \\
\hline & & & & SSE & $\$ 0.00$ & 30 & years \\
\hline & & & & ESE & $\$ 0.00$ & 30 & years \\
\hline & & & & ENE & $\$ 0.00$ & 30 & years \\
\hline & & & & NNE & $\$ 0.00$ & 30 & years \\
\hline & & & & NNW & $\$ 0.00$ & 30 & years \\
\hline & & & & WNW & $\$ 0.00$ & 30 & years \\
\hline & & & & WSW & $\$ 0.00$ & 30 & years \\
\hline & & & & SSW & $\$ 0.00$ & 30 & years \\
\hline \multicolumn{8}{|l|}{ Building } \\
\hline & Neighbors & & & & & & \\
\hline & & & & No Neighbors & $\$ 0.00$ & 30 & years \\
\hline & & & & at 20ft & $\$ 0.00$ & 30 & years \\
\hline & & $x$ & $x$ & at $15 \mathrm{ft}$ & $\$ 0.00$ & 30 & years \\
\hline & & & & at $10 \mathrm{ft}$ & $\$ 0.00$ & 30 & years \\
\hline \multicolumn{8}{|l|}{ Building } \\
\hline & Aspect Ratio & & & & & & \\
\hline & & & & 1.5 & $\$ 0.00$ & 30 & years \\
\hline & & $x$ & $x$ & 1.33 & $\$ 0.00$ & 30 & years \\
\hline & & & & 1 & $\$ 0.00$ & 30 & years \\
\hline & & & & 0.75 & $\$ 0.00$ & 30 & years \\
\hline & & & & 0.67 & $\$ 0.00$ & 30 & years \\
\hline \multicolumn{8}{|l|}{\begin{tabular}{|l} 
Building \\
\end{tabular}} \\
\hline & Misc Electric Loads & & & & & & 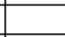 \\
\hline & & & & 4 & $\$ 0.00$ & 30 & years \\
\hline & & & & 2 & $\$ 0.00$ & 30 & years \\
\hline & & & & 1.5 & $\$ 0.00$ & 30 & years \\
\hline & & $x$ & $x$ & 1 & $\$ 0.00$ & 30 & years \\
\hline & & & & 0.75 & $\$ 0.00$ & 30 & years \\
\hline & & & & 0.5 & $\$ 0.00$ & 30 & years \\
\hline & & & & 0.25 & $\$ 0.00$ & 30 & years \\
\hline \multicolumn{8}{|l|}{ Building } \\
\hline & Misc Gas Loads & & & & & & 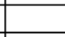 \\
\hline & & & & 2 & $\$ 0.00$ & 30 & years \\
\hline & & $x$ & $x$ & 1 & $\$ 0.00$ & 30 & years \\
\hline & & & & 0.5 & $\$ 0.00$ & 30 & years \\
\hline & & & & 0 & $\$ 0.00$ & 30 & years \\
\hline \multicolumn{8}{|l|}{ Building } \\
\hline & Heating Set Point & & & & & & \\
\hline & & $x$ & $x$ & $68 \mathrm{~F}$ & $\$ 0.00$ & 30 & years \\
\hline & & & & $69 \mathrm{~F}$ & $\$ 0.00$ & 30 & years \\
\hline & & & & $70 \mathrm{~F}$ & $\$ 0.00$ & 30 & years \\
\hline & & & & $71 \mathrm{~F}$ & $\$ 0.00$ & 30 & years \\
\hline & & & & $72 \mathrm{~F}$ & $\$ 0.00$ & 30 & years \\
\hline & & & & $73 \mathrm{~F}$ & $\$ 0.00$ & 30 & years \\
\hline & & & & $74 \mathrm{~F}$ & $\$ 0.00$ & 30 & years \\
\hline & & & & $75 \mathrm{~F}$ & $\$ 0.00$ & 30 & years \\
\hline & & & & $71 \mathrm{~F} \mathrm{w} /$ setback $65 \mathrm{~F}$ & $\$ 0.00$ & 30 & years \\
\hline & & & & $71 \mathrm{~F}$ w/ setback $65 \mathrm{~F}$ (wkdy) & $\$ 0.00$ & 30 & years \\
\hline \multicolumn{8}{|l|}{ Building } \\
\hline & Cooling Set Point & & & & & & \\
\hline & & & & $73 \mathrm{~F}$ & $\$ 0.00$ & 30 & years \\
\hline & & & & $74 \mathrm{~F}$ & $\$ 0.00$ & 30 & years \\
\hline & & & & $75 \mathrm{~F}$ & $\$ 0.00$ & 30 & years \\
\hline & & & & $76 \mathrm{~F}$ & $\$ 0.00$ & 30 & years \\
\hline & & & & $77 \mathrm{~F}$ & $\$ 0.00$ & 30 & years \\
\hline & & $x$ & $x$ & $78 \mathrm{~F}$ & $\$ 0.00$ & 30 & years \\
\hline & & & & $79 \mathrm{~F}$ & $\$ 0.00$ & 30 & years \\
\hline & & & & $80 \mathrm{~F}$ & $\$ 0.00$ & 30 & years \\
\hline
\end{tabular}




\begin{tabular}{|c|c|c|c|c|c|c|c|}
\hline GROUP & CATEGORY & REF & SELECTED & OPTION NAME & UNIT COST & LIFETIME & \\
\hline & & & & $76 \mathrm{~F} \mathrm{w} /$ setup $85 \mathrm{~F}$ & $\$ 0.00$ & 30 & years \\
\hline & & & & $76 \mathrm{~F} \mathrm{w} /$ setup $81 \mathrm{~F}$ & $\$ 0.00$ & 30 & years \\
\hline \multicolumn{8}{|l|}{ Building } \\
\hline & Ventilation Rate & & & & & & \\
\hline & & & & None & $\$$ & 0 & years \\
\hline & & & & Spot ventilation only & $\$$ & 0 & years \\
\hline & & & & $50 \%$ ASHRAE 62.2 & $\$$ & 0 & years \\
\hline & & & & $60 \%$ ASHRAE 62.2 & $\$$ & 0 & years \\
\hline & & & & $70 \%$ ASHRAE 62.2 & $\$$ & 0 & years \\
\hline & & & & $80 \%$ ASHRAE 62.2 & $\$$ & 0 & years \\
\hline & & & & $90 \%$ ASHRAE 62.2 & $\$$ & 0 & years \\
\hline & & $x$ & $x$ & $100 \%$ ASHRAE 62.2 & $\$$ & 0 & years \\
\hline & & & & $110 \%$ ASHRAE 62.2 & $\$$ & 0 & years \\
\hline & & & & $120 \%$ ASHRAE 62.2 & $\$$ & 0 & years \\
\hline & & & & $130 \%$ ASHRAE 62.2 & $\$$ & 0 & years \\
\hline & & & & $140 \%$ ASHRAE 62.2 & $\$$ & 0 & years \\
\hline & & & & $150 \%$ ASHRAE 62.2 & $\$$ & 0 & years \\
\hline \multicolumn{8}{|l|}{ Building } \\
\hline & Natural Ventilation & & & & & & \\
\hline & & & & None & $\$ 0.00$ & 30 & years \\
\hline & & $x$ & $x$ & Benchmark & $\$ 0.00$ & 30 & years \\
\hline & & & & Smart & $\$ 0.00$ & 30 & years \\
\hline \multicolumn{8}{|l|}{ Envelope } \\
\hline & Wall Insulation & & & & & & \\
\hline & & & & R11 batts, $2 \times 4,16$ "o.c. & $\$ 6.61$ & 30 & years \\
\hline & & $x$ & $x$ & R13 batts, $2 \times 4,16$ "o.c. & $\$ 6.65$ & 30 & years \\
\hline & & & $x$ & R15 batts, $2 \times 4,16 "$ o.c. & $\$ 6.69$ & 30 & years \\
\hline & & & & R19 batts, $2 \times 6,24$ "o.c. & $\$ 6.78$ & 30 & years \\
\hline & & & & R21 batts, $2 \times 6,24$ "o.c. & $\$ 6.81$ & 30 & years \\
\hline & & & & R11 batts, $2 \times 4,16$ "o.c. +1 " foam & $\$ 7.45$ & 30 & years \\
\hline & & & & R13 batts, $2 \times 4,16 "$ o.c. +1 " foam & $\$ 7.49$ & 30 & years \\
\hline & & & & R19 batts, $2 \times 6,24$ "o.c. +1 " foam & $\$ 7.61$ & 30 & years \\
\hline & & & & R21 batts, $2 \times 6,24$ "o.c. +1 " foam & $\$ 7.65$ & 30 & years \\
\hline & & & & R19 batts, $2 \times 6,24$ "o.c. +2 " foam & $\$ 8.09$ & 30 & years \\
\hline & & & & 2-Stud, R33 2x4 24" o.c., 12" total & $\$ 8.46$ & 30 & years \\
\hline & & & & 2-Stud, R39 2x4 24" o.c., 12" total & $\$ 8.58$ & 30 & years \\
\hline & & & & 2-Stud, R45 2x4 24" o.c., 12" total & $\$ 8.70$ & 30 & years \\
\hline & & & & 2-Stud, R51 $2 \times 424 "$ o.c., 14 " total & $\$ 8.80$ & 30 & years \\
\hline & & & & $4.5^{\prime \prime}$ SIPS (3.5" Core) & $\$ 8.21$ & 30 & years \\
\hline & & & & $6.5^{\prime \prime}$ SIPS (5.5" Core) & $\$ 9.12$ & 30 & years \\
\hline & & & & $8.5^{\prime \prime}$ SIPS (7.5" Core) & $\$ 10.02$ & 30 & years \\
\hline & & & & 10.5" SIPs (9.5" Core) & $\$ 11.25$ & 30 & years \\
\hline \multicolumn{8}{|l|}{ Envelope } \\
\hline & Ceiling Insulation & & & & & & \\
\hline & & & & R30 Cellulose & $\$ 0.79$ & 30 & years \\
\hline & & & & R40 Cellulose & $\$ 1.02$ & 30 & years \\
\hline & & & & R50 Cellulose & $\$ 1.30$ & 30 & years \\
\hline & & & & R60 Cellulose & $\$ 1.53$ & 30 & years \\
\hline & & $x$ & $x$ & R30 Fiberglass & $\$ 1.20$ & 30 & years \\
\hline & & & $x$ & R40 Fiberglass & $\$ 1.55$ & 30 & years \\
\hline & & & $x$ & R50 Fiberglass & $\$ 1.89$ & 30 & years \\
\hline & & & $x$ & R60 Fiberglass & $\$ 2.32$ & 30 & years \\
\hline \multicolumn{8}{|l|}{ Envelope } \\
\hline & Garage Ceiling & & & & & & \\
\hline & & & & None & $\$ 0.00$ & 30 & years \\
\hline & & & & R11 Fiberglass & $\$ 0.43$ & 30 & years \\
\hline & & & & R19 Fiberglass & $\$ 0.61$ & 30 & years \\
\hline & & $x$ & $x$ & R30 Fiberglass & $\$ 0.94$ & 30 & years \\
\hline \multicolumn{8}{|l|}{ Envelope } \\
\hline & Roofing Material & & & & & & \\
\hline & & & & Asphalt Shingles, Dark & $\$ 1.25$ & 25 & years \\
\hline & & $x$ & $x$ & Asphalt Shinges, Medium & $\$ 1.25$ & 25 & years \\
\hline & & & & Asphalt Shingles, Light & $\$ 1.25$ & 25 & years \\
\hline & & & $x$ & Asphalt Shingles, White or cool colors & $\$ 1.25$ & 25 & years \\
\hline & & & & Tile, Dark & $\$ 4.23$ & 30 & years \\
\hline & & & & Tile, Medium (mottled, terra cotta, buff) & $\$ 4.23$ & 30 & years \\
\hline & & & & Tile, Light & $\$ 4.23$ & 30 & years \\
\hline & & & & Tile, White & $\$ 4.23$ & 30 & years \\
\hline
\end{tabular}




\begin{tabular}{|c|c|c|c|c|c|c|c|}
\hline GROUP & CATEGORY & REF & SELECTED & OPTION NAME & UNIT COST & LIFETIME & \\
\hline & & & & Metal, Dark & $\$ 2.87$ & 30 & years \\
\hline & & & & Metal, Medium & $\$ 2.87$ & 30 & years \\
\hline & & & & Metal, Light & $\$ 2.87$ & 30 & years \\
\hline & & & & Metal, White & $\$ 2.87$ & 30 & years \\
\hline & & & & Galvanized Steel & $\$ 1.94$ & 30 & years \\
\hline & & & & Gavalume Steel & $\$ 2.47$ & 30 & years \\
\hline \multicolumn{8}{|c|}{ Envelope } \\
\hline & Radiant Barrier & & & & & & \\
\hline & & $x$ & $x$ & None & $\$ 0.00$ & 30 & years \\
\hline & & & $x$ & Radiant Barrier & $\$ 0.32$ & 30 & years \\
\hline \multicolumn{8}{|l|}{ Envelope } \\
\hline & Infiltration & & & & & & \\
\hline & & $x$ & & Typical & $\$ 0.00$ & 13 & years \\
\hline & & & $x$ & Tight & $\$ 0.54$ & 13 & years \\
\hline & & & $x$ & Tighter & $\$ 1.08$ & 13 & years \\
\hline & & & $x$ & Tightest & $\$ 1.62$ & 13 & years \\
\hline \multicolumn{8}{|c|}{ Foundation } \\
\hline & Slab & & & & & & \\
\hline & & & & No Slab & $\$ 0.00 \mid \$ 0.00$ & 30 & years \\
\hline & & $\mathrm{x}$ & $x$ & Uninsulated & $\$ 0.00 \mid \$ 0.00$ & 30 & years \\
\hline & & & $x$ & 2ft R5 Perimeter, R5 Gap & $\$ 1.01 \mid \$ 1.01$ & 30 & years \\
\hline & & & & 4ft R5 Perimeter, R5 Gap & $\$ 1.01 \mid \$ 1.01$ & 30 & years \\
\hline & & & & 2ft R10 Perimeter, R5 Gap & $\$ 1.64 \mid \$ 1.01$ & 30 & years \\
\hline & & & & 4ft R10 Perimeter, R5 Gap & $\$ 1.64 \mid \$ 1.01$ & 30 & years \\
\hline & & & & 15ft R10 Perimeter, R5 Gap & $\$ 1.64 \mid \$ 1.01$ & 30 & years \\
\hline \multicolumn{8}{|c|}{ Foundation } \\
\hline & Basement & & & & & & \\
\hline & & $x$ & $x$ & No Basement & $\$ 0.00 \mid \$$ & 30 & years \\
\hline & & & & Uninsulated & $\$ 0.00 \mid \$ 0.00$ & 30 & years \\
\hline & & & & 4ft R5 Exterior & $\$ 1.01 \mid \$ 0.00$ & 30 & years \\
\hline & & & & 4ft R10 Exterior & $\$ 1.64 \mid \$ 0.00$ & 30 & years \\
\hline & & & & 8ft R10 Exterior & $\$ 1.64 \mid \$ 0.00$ & 30 & years \\
\hline & & & & 8ft R15 Exterior & $\$ 2.04 \mid \$ 0.00$ & 30 & years \\
\hline & & & & 8ft R20 Exterior & $\$ 2.30 \mid \$ 0.00$ & 30 & years \\
\hline & & & & $8 \mathrm{ft}$ R10 Interior & $\$ 1.64 \mid \$ 0.00$ & 30 & years \\
\hline & & & & Uninsulated Wood Frame & $\$ 0.13 \mid \$ 0.00$ & 30 & years \\
\hline & & & & R11 Wood Frame & $\$ 1.15 \mid \$ 0.00$ & 30 & years \\
\hline & & & & R19 Wood Frame & $\$ 1.33 \mid \$ 0.00$ & 30 & years \\
\hline & & & & R30 Wood Frame & $\$ 1.80 \mid \$ 0.00$ & 30 & years \\
\hline & & & & R11 Ceiling & $\$ 0.00 \mid \$ 1.16$ & 30 & years \\
\hline & & & & R19 Ceiling & $\$ 0.00 \mid \$ 1.63$ & 30 & years \\
\hline \multicolumn{8}{|c|}{ Foundation } \\
\hline & Crawl Space & & & & & & 7 \\
\hline & & $x$ & $x$ & No Crawl Space & $\$ 0.00 \mid \$ 0.00$ & 30 & years \\
\hline & & & & Unvented, Uninsulated & $\$ 0.00 \mid \$ 0.00$ & 30 & years \\
\hline & & & & Unvented, R10 Interior & $\$ 1.15 \mid \$ 0.00$ & 30 & years \\
\hline & & & & Vented, R19 Ceiling & $\$ 0.00 \mid \$ 1.16$ & 30 & years \\
\hline & & & & Vented, R30 Ceiling & $\$ 0.00 \mid \$ 1.63$ & 30 & years \\
\hline \multicolumn{8}{|c|}{ Thermal Mass } \\
\hline & Exposed Floor & & & & & & \\
\hline & & & & No Exposed Floor & $\$ 0.00 \mid \$ 0.00$ & 30 & years \\
\hline & & $x$ & $x$ & $20 \%$ Exposed & $\$ 0.00 \mid \$ 0.00$ & 30 & years \\
\hline & & & & $40 \%$ Exposed & $\$ 0.00 \mid \$ 0.00$ & 30 & years \\
\hline & & & & $60 \%$ Exposed & $\$ 0.00 \mid \$ 0.00$ & 30 & years \\
\hline & & & & $80 \%$ Exposed & $\$ 0.00 \mid \$ 0.00$ & 30 & years \\
\hline & & & & $100 \%$ Exposed & $\$ 0.00 \mid \$ 0.00$ & 30 & years \\
\hline \multicolumn{8}{|c|}{ Thermal Mass } \\
\hline & Ceiling Mass & & & & & & \\
\hline & & $x$ & $x$ & 1/2" Ceiling Drywall & $\$ 0.60$ & 30 & years \\
\hline & & & & 5/8" Ceiling Drywall & $\$ 0.62$ & 30 & years \\
\hline & & & & $2 \times 1 / 2 "$ Ceiling Drywall & $\$ 1.10$ & 30 & years \\
\hline & & & & $2 \times 5 / 8$ " Ceiling Drywall & $\$ 1.14$ & 30 & years \\
\hline \multicolumn{8}{|c|}{ Thermal Mass } \\
\hline & Wall Mass & & & & & & \\
\hline & & $x$ & $x$ & Exterior and Partition, 1/2" Drywall & $\$ 0.60 \mid \$ 0.60$ & 30 & years \\
\hline & & & & Exterior, 5/8" Drywall & $\$ 0.62 \mid \$ 0.60$ & 30 & years \\
\hline & & & & Exterior, $2 \times 1 / 2 "$ Drywall & $\$ 1.10 \mid \$ 0.60$ & 30 & years \\
\hline & & & & Exterior, 2 x 5/8" Drywall & $\$ 1.14 \mid \$ 0.60$ & 30 & years \\
\hline
\end{tabular}




\begin{tabular}{|c|c|c|c|c|c|c|c|}
\hline GROUP & CATEGORY & REF & SELECTED & OPTION NAME & UNIT COST & LIFETIME & \\
\hline & & & & Partition, 5/8" Drywall & $\$ 0.60 \mid \$ 0.62$ & 30 & years \\
\hline & & & & Partition, $2 \times 1 / 2$ " Drywall & $\$ 0.60 \mid \$ 1.10$ & 30 & years \\
\hline & & & & Partition, 2 x 5/8" Drywall & $\$ 0.60 \mid \$ 1.14$ & 30 & years \\
\hline & & & & Exterior and Partition, 5/8" Drywall & $\$ 0.62 \mid \$ 0.62$ & 30 & years \\
\hline & & & & Exterior and Partition, $2 \times 1 / 2 "$ Drywall & $\$ 1.10 \mid \$ 1.10$ & 30 & years \\
\hline & & & & Exterior and Partition, 2 x 5/8" Drywall & $\$ 1.14 \mid \$ 1.14$ & 30 & years \\
\hline \multicolumn{8}{|c|}{ Windows \& Shading } \\
\hline & Window Areas & & & & & & \\
\hline & & & & $20.0 \% \quad$ F25 B25 L25 R25 & $\$$ & 0 & years \\
\hline & & & & $20.0 \% \quad \mathrm{~F} 20 \mathrm{~B} 40 \mathrm{~L} 20 \mathrm{R} 20$ & $\$$ & 0 & years \\
\hline & & $\mathrm{x}$ & $x$ & $18.0 \% \quad$ F25 B25 L25 R25 & $\$$ & 0 & years \\
\hline & & & & $18.0 \%$ F20 B40 L20 R20 & $\$$ & 0 & years \\
\hline & & & & $16.0 \% \quad$ F25 B25 L25 R25 & $\$$ & 0 & years \\
\hline & & & & $16.0 \%$ F20 B40 L20 R20 & $\$$ & 0 & years \\
\hline \multicolumn{8}{|c|}{ Windows \& Shading } \\
\hline & Window Type & & & & & & \\
\hline & & & & Single Pane & $\$ 4.66$ & 20 & years \\
\hline & & & & Double Clear & $\$ 14.00$ & 20 & years \\
\hline & & & $x$ & Low-e low SHGC arg & $\$ 16.00$ & 20 & years \\
\hline & & & & Low-e std SHGC arg & $\$ 16.00$ & 20 & years \\
\hline & & & & Low-e high SHGC arg & $\$ 16.00$ & 20 & years \\
\hline & & & & Low-e v. high SHGC arg & $\$ 16.00$ & 20 & years \\
\hline & & & & 3 pane, $1 \mathrm{HM}$ & $\$ 18.00$ & 20 & years \\
\hline & & & & 4 pane, $2 \mathrm{HM} \mathrm{Kr}$ & $\$ 24.00$ & 20 & years \\
\hline & & $x$ & $x$ & Low-e, low SHGC & $\$ 16.00$ & 20 & years \\
\hline & & & & Low-e std. SHGC & $\$ 16.00$ & 20 & years \\
\hline & & & & Low-e high SHGC & $\$ 16.00$ & 20 & years \\
\hline & & & & Low-e v. high SHGC & $\$ 16.00$ & 20 & years \\
\hline \multicolumn{8}{|c|}{ Windows \& Shading } \\
\hline & Eaves & & & & & & \\
\hline & & & & No eaves & $\$ 10.65$ & 30 & years \\
\hline & & $x$ & $x$ & $1 \mathrm{ft}$ overhang & $\$ 10.65$ & 30 & years \\
\hline & & & $x$ & $2 \mathrm{ft}$ overhang & $\$ 10.65$ & 30 & years \\
\hline & & & $x$ & $3 \mathrm{ft}$ overhang & $\$ 10.65$ & 30 & years \\
\hline \multicolumn{8}{|c|}{\begin{tabular}{|l} 
Lg. Appliances \\
\end{tabular}} \\
\hline & Refrigerator & & & & & & \\
\hline & & $x$ & $x$ & Standard & $\$ 1,100.00$ & 18 & years \\
\hline & & & & EnergyStar & $\$ 1,220.00$ & 18 & years \\
\hline \multicolumn{8}{|c|}{\begin{tabular}{|l} 
Lg. Appliances \\
\end{tabular}} \\
\hline & Cooking Range & & & & & & \\
\hline & & & & Electric & $\$ 350.00$ & 13 & years \\
\hline & & $x$ & $x$ & Gas & $\$ 350.00$ & 15 & years \\
\hline \multicolumn{8}{|c|}{\begin{tabular}{|l} 
Lg. Appliances \\
\end{tabular}} \\
\hline & Dishwasher & & & & & & \\
\hline & & $\mathrm{x}$ & $x$ & Standard & $\$ 259.00$ & 13 & years \\
\hline & & & & EnergyStar & $\$ 329.00$ & 13 & years \\
\hline \multicolumn{8}{|c|}{ Lg. Appliances } \\
\hline & Clothes Dryer & & & & & & \\
\hline & & & & Clothes Line & $\$ 0.01$ & 30 & years \\
\hline & & $x$ & $x$ & Electric & $\$ 269.00$ & 18 & years \\
\hline & & & & Gas & $\$ 319.00$ & 18 & years \\
\hline \multicolumn{8}{|c|}{\begin{tabular}{|l} 
Lg. Appliances \\
\end{tabular}} \\
\hline & Clothes Washer & & & & & & \\
\hline & & $x$ & $x$ & Standard (V-Axis) & $\$ 419.00$ & 14 & years \\
\hline & & & & EnergyStar (H-Axis) & $\$ 799.00$ & 14 & years \\
\hline & & & & Standard (V-Axis) - Cold Only & $\$ 419.00$ & 14 & years \\
\hline & & & & EnergyStar (H-Axis) - Cold Only & $\$ 799.00$ & 14 & years \\
\hline \multicolumn{8}{|l|}{ Lighting } \\
\hline & Hardwired Lighting & & & & & & \\
\hline & & $x$ & $x$ & $14 \%$ Fluorescent & $\$ 0.42 \mid \$ 3.79$ & 1.33 & years \\
\hline & & & & $20 \%$ Fluorescent & $\$ 0.42 \mid \$ 3.79$ & 1.66 & years \\
\hline & & & & $30 \%$ Fluorescent & $\$ 0.42 \mid \$ 3.79$ & 2.46 & years \\
\hline & & & & $40 \%$ Fluorescent & $\$ 0.42 \mid \$ 3.79$ & 3.25 & years \\
\hline & & & $x$ & $50 \%$ Fluorescent & $\$ 0.42 \mid \$ 3.79$ & 4.03 & years \\
\hline & & & & $60 \%$ Fluorescent & $\$ 0.42 \mid \$ 3.79$ & 4.81 & years \\
\hline & & & & $70 \%$ Fluorescent & $\$ 0.42 \mid \$ 3.79$ & 5.59 & years \\
\hline & & & & $80 \%$ Fluorescent & $\$ 0.42 \mid \$ 3.79$ & 6.39 & years \\
\hline & & & & $90 \%$ Fluorescent & $\$ 0.42 \mid \$ 3.79$ & 7.19 & years \\
\hline
\end{tabular}




\begin{tabular}{|c|c|c|c|c|c|c|c|}
\hline GROUP & CATEGORY & REF & SELECTED & OPTION NAME & UNIT COST & LIFETIME & \\
\hline & & & $x$ & $100 \%$ Fluorescent & $\$ 0.42 \mid \$ 3.79$ & 8.01 & years \\
\hline \multicolumn{8}{|l|}{ Lighting } \\
\hline & Plug-in Lighting & & & & & & \\
\hline & & $x$ & $x$ & $0 \%$ CFL & $\$ 0.42 \mid \$ 3.79$ & 1.33 & years \\
\hline & & & & $10 \%$ CFL & $\$ 0.42 \mid \$ 3.79$ & 1.92 & years \\
\hline & & & & $20 \% \mathrm{CFL}$ & $\$ 0.42 \mid \$ 3.79$ & 2.57 & years \\
\hline & & & & $30 \% \mathrm{CFL}$ & $\$ 0.42 \mid \$ 3.79$ & 3.23 & years \\
\hline & & & & $40 \%$ CFL & $\$ 0.42 \mid \$ 3.79$ & 3.89 & years \\
\hline & & & & $50 \% \mathrm{CFL}$ & $\$ 0.42 \mid \$ 3.79$ & 4.55 & years \\
\hline & & & & $60 \% \mathrm{CFL}$ & $\$ 0.42 \mid \$ 3.79$ & 5.22 & years \\
\hline & & & & $70 \%$ CFL & $\$ 0.42 \mid \$ 3.79$ & 5.9 & years \\
\hline & & & & $80 \% \mathrm{CFL}$ & $\$ 0.42 \mid \$ 3.79$ & 6.59 & years \\
\hline & & & & $90 \% \mathrm{CFL}$ & $\$ 0.42 \mid \$ 3.79$ & 7.3 & years \\
\hline & & & & $100 \%$ CFL & $\$ 0.42 \mid \$ 3.79$ & 8.01 & years \\
\hline \multicolumn{8}{|l|}{ Equipment } \\
\hline & Air Conditioner & & & & & & \\
\hline & & & & No Air Conditioner & $\$ 0.00$ & 18 & years \\
\hline & & & & SEER 10 & $\$ 266.00$ & 18 & years \\
\hline & & $x$ & $x$ & SEER 13 & $\$ 721.00$ & 18 & years \\
\hline & & & $x$ & SEER 14 & $\$ 873.00$ & 18 & years \\
\hline & & & $x$ & SEER 15 & $\$ 1,025.00$ & 18 & years \\
\hline & & & $x$ & SEER 16 & $\$ 1,177.00$ & 18 & years \\
\hline & & & $x$ & SEER 17 & $\$ 1,329.00$ & 18 & years \\
\hline & & & $x$ & SEER 18 & $\$ 1,481.00$ & 18 & years \\
\hline \multicolumn{8}{|l|}{ Equipment } \\
\hline & Furnace & & & & & & \\
\hline & & & & No Furnace & $\$ 0.00$ & 18 & years \\
\hline & & $x$ & $x$ & AFUE $80 \%$ & $\$ 265.00$ & 18 & years \\
\hline & & & $x$ & AFUE $92.5 \%$ & $\$ 559.00$ & 18 & years \\
\hline \multicolumn{8}{|l|}{ Equipment } \\
\hline & Heat Pump & & & & & & \\
\hline & & $x$ & $x$ & No Heat Pump & $\$ 0.00$ & 15 & years \\
\hline & & & & SEER 10. HSPF 7.2 & $\$ 555.00$ & 15 & years \\
\hline & & & & SEER 13. HSPF 8.1 & $\$ 1,216.00$ & 15 & years \\
\hline & & & & SEER 14. HSPF 8.6 & $\$ 1,436.00$ & 15 & years \\
\hline & & & & SEER 15. HSPF 8.8 & $\$ 1,656.00$ & 15 & years \\
\hline & & & & SEER 16. HSPF 8.4 & $\$ 1,876.00$ & 15 & years \\
\hline & & & & SEER 17. HSPF 8.6 & $\$ 2,096.00$ & 15 & years \\
\hline & & & & SEER 18. HSPF 9.2 & $\$ 2,316.00$ & 15 & years \\
\hline \multicolumn{8}{|l|}{ Equipment } \\
\hline & Mechanical Ventilation & & & & & & \\
\hline & & & & None & $\$ 0.00$ & 20 & years \\
\hline & & $x$ & $x$ & Upgraded Bathroom Exhaust & $\$ 463.00$ & 20 & years \\
\hline & & & & Balanced Energy-Recovery Ventilator & $\$ 1,838.00$ & 20 & years \\
\hline \multicolumn{8}{|l|}{ Equipment } \\
\hline & Water Heater & & & & & & \\
\hline & & & & Electric Standard & $\$ 479.00$ & 15 & years \\
\hline & & & & Electric Premium & $\$ 570.00$ & 15 & years \\
\hline & & & & Electric Tankless & $\$ 1,075.00$ & 20 & years \\
\hline & & $x$ & $x$ & Gas Standard & $\$ 428.00$ & 13 & years \\
\hline & & & $x$ & Gas Premium & $\$ 624.00$ & 13 & years \\
\hline & & & $\mathrm{x}$ & Gas Tankless & $\$ 1,050.00$ & 20 & years \\
\hline \multicolumn{8}{|l|}{ Equipment } \\
\hline & Ducts & & & & & & \\
\hline & & & & None & $\$ 0.00$ & 30 & years \\
\hline & & $x$ & $x$ & Typical & $\$ 0.45$ & 18 & years \\
\hline & & & $x$ & Improved & $\$ 0.69$ & 18 & years \\
\hline & & & $x$ & Inside & $\$ 0.77$ & 18 & years \\
\hline \multicolumn{8}{|c|}{ Renewables } \\
\hline & Solar DHW & & & & & & \\
\hline & & $x$ & $x$ & No Solar DHW & $\$ 0.00$ & 30 & years \\
\hline & & & $x$ & $32 \mathrm{sq} \mathrm{ft} \mathrm{ICS}$ & $\$ 2,654.00$ & 30 & years \\
\hline & & & & 40 sq ft closed loop & $\$ 4,307.00$ & 30 & years \\
\hline & & & $x$ & $64 \mathrm{sq} \mathrm{ft}$ closed loop & $\$ 4,768.00$ & 30 & years \\
\hline \multicolumn{8}{|l|}{ Renewables } \\
\hline & SDHW Azimuth & & & & & & \\
\hline & & & & Back Roof & $\$$ & 0 & years \\
\hline & & & & Front Roof & $\$$ & 0 & years \\
\hline
\end{tabular}




\begin{tabular}{|c|c|c|c|c|c|c|c|}
\hline GROUP & CATEGORY & REF & SELECTED & OPTION NAME & UNIT COST & LIFETIME & \\
\hline & & & & Left Roof & $\$$ & 0 & years \\
\hline & & & & Right Roof & $\$$ & 0 & years \\
\hline & & & & West & $\$$ & 0 & years \\
\hline & & & & Southwest & $\$$ & 0 & years \\
\hline & & $x$ & $x$ & South & $\$$ & 0 & years \\
\hline & & & & Southeast & $\$$ & 0 & years \\
\hline & & & & East & $\$$ & 0 & years \\
\hline \multicolumn{8}{|c|}{ Renewables } \\
\hline & SDHW Tilt & & & & & & \\
\hline & & & & Roof Pitch & $\$$ & 0 & years \\
\hline & & & & $0^{\circ}$ & $\$$ & 0 & years \\
\hline & & & & $10^{\circ}$ & $\$$ & 0 & years \\
\hline & & & & $20^{\circ}$ & $\$$ & 0 & years \\
\hline & & & & $30^{\circ}$ & $\$$ & 0 & years \\
\hline & & & & $40^{\circ}$ & $\$$ & 0 & years \\
\hline & & & & $50^{\circ}$ & $\$$ & 0 & years \\
\hline & & & & $60^{\circ}$ & $\$$ & 0 & years \\
\hline & & & & $70^{\circ}$ & $\$$ & 0 & years \\
\hline & & & & $80^{\circ}$ & $\$$ & 0 & years \\
\hline & & & & $90^{\circ}$ & $\$$ & 0 & years \\
\hline & & & & Latitude $-15^{\circ}$ & $\$$ & 0 & years \\
\hline & & $x$ & $x$ & Latitude & $\$$ & 0 & years \\
\hline & & & & Latitude $+15^{\circ}$ & $\$$ & 0 & years \\
\hline \multicolumn{8}{|c|}{ Renewables } \\
\hline & PV Size & & & & & & \\
\hline & & $\mathrm{x}$ & $x$ & $0 \mathrm{~kW}$ & $\$$ & 0 & years \\
\hline & & & & $0.5 \mathrm{~kW}$ & $\$$ & 0 & years \\
\hline & & & $x$ & $1.0 \mathrm{~kW}$ & $\$$ & 0 & years \\
\hline & & & & $1.5 \mathrm{~kW}$ & $\$$ & 0 & years \\
\hline & & & $x$ & $2.0 \mathrm{~kW}$ & $\$$ & 0 & years \\
\hline & & & & $2.5 \mathrm{~kW}$ & $\$$ & 0 & years \\
\hline & & & $x$ & $3.0 \mathrm{~kW}$ & $\$$ & 0 & years \\
\hline & & & & $3.5 \mathrm{~kW}$ & $\$$ & 0 & years \\
\hline & & & $x$ & $4.0 \mathrm{~kW}$ & $\$$ & 0 & years \\
\hline & & & & $4.5 \mathrm{~kW}$ & $\$$ & 0 & years \\
\hline & & & $x$ & $5.0 \mathrm{~kW}$ & $\$$ & 0 & years \\
\hline & & & & $5.5 \mathrm{~kW}$ & $\$$ & 0 & years \\
\hline & & & & $6.0 \mathrm{~kW}$ & $\$$ & 0 & years \\
\hline & & & & $6.5 \mathrm{~kW}$ & $\$$ & 0 & years \\
\hline & & & & $7.0 \mathrm{~kW}$ & $\$$ & 0 & years \\
\hline & & & & $7.5 \mathrm{~kW}$ & $\$$ & 0 & years \\
\hline & & & & $8.0 \mathrm{~kW}$ & $\$$ & 0 & years \\
\hline & & & & ZNE & $\$$ & 0 & years \\
\hline \multicolumn{8}{|c|}{ Renewables } \\
\hline & PV Azimuth & & & & & & 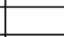 \\
\hline & & & & Back Roof & $\$$ & 0 & years \\
\hline & & & & Front Roof & $\$$ & 0 & years \\
\hline & & & & Left Roof & $\$$ & 0 & years \\
\hline & & & & Right Roof & $\$$ & 0 & years \\
\hline & & & & West & $\$$ & 0 & years \\
\hline & & & & Southwest & $\$$ & 0 & years \\
\hline & & $x$ & $x$ & South & $\$$ & 0 & years \\
\hline & & & & Southeast & $\$$ & 0 & years \\
\hline & & & & East & $\$$ & 0 & years \\
\hline \multicolumn{8}{|c|}{ Renewables } \\
\hline & PV Tilt & & & & & & \\
\hline & & & & Roof Pitch & $\$$ & 0 & years \\
\hline & & & & $0^{\circ}$ & $\$$ & 0 & years \\
\hline & & & & $10^{\circ}$ & $\$$ & 0 & years \\
\hline & & & & $20^{\circ}$ & $\$$ & 0 & years \\
\hline & & & & $30^{\circ}$ & $\$$ & 0 & years \\
\hline & & & & $40^{\circ}$ & $\$$ & 0 & years \\
\hline & & & & $50^{\circ}$ & $\$$ & 0 & years \\
\hline & & & & $60^{\circ}$ & $\$$ & 0 & years \\
\hline & & & & $70^{\circ}$ & $\$$ & 0 & years \\
\hline & & & & $80^{\circ}$ & $\$$ & 0 & years \\
\hline & & & & $90^{\circ}$ & $\$$ & 0 & years \\
\hline & & & & Latitude $-15^{\circ}$ & $\$$ & 0 & years \\
\hline
\end{tabular}




\begin{tabular}{|c|c|c|c|c|c|c|c|}
\hline GROUP & CATEGORY & REF & SELECTED & OPTION NAME & UNIT COST & LIFETIME & \\
\hline & & $x$ & $x$ & Latitude & $\$$ & 0 & years \\
\hline & & & & Latitude $+15^{\circ}$ & $\$$ & 0 & years \\
\hline \multicolumn{8}{|l|}{ HVAC Sizing } \\
\hline & Cooling Capacity & & & & & & \\
\hline & & & $x$ & 0 tons & $\$ 0.00 \mid \$ 0.00$ & 0 & years \\
\hline & & & $x$ & 1.5 tons & $\$ 690.00 \mid \$ 554.00$ & 0 & years \\
\hline & & & $\mathrm{x}$ & 2.0 tons & $\$ 920.00 \mid \$ 738.00$ & 0 & years \\
\hline & & & $x$ & 2.5 tons & $\$ 1,150.00 \mid \$ 923.00$ & 0 & years \\
\hline & & & $x$ & 3.0 tons & $\$ 1,380.00 \mid \$ 1,107.00$ & 0 & years \\
\hline & & & $x$ & 3.5 tons & $\$ 1,610.00 \mid \$ 1,292.00$ & 0 & years \\
\hline & & & $x$ & 4.0 tons & $\$ 1,840.00 \mid \$ 1,476.00$ & 0 & years \\
\hline & & & $x$ & 5.0 tons & $\$ 2,300.00 \mid \$ 1,845.00$ & 0 & years \\
\hline \multicolumn{8}{|l|}{ HVAC Sizing } \\
\hline & Heating Capacity & & & & & & \\
\hline & & & $x$ & $0 \mathrm{kBtu} / \mathrm{hr}$ & $\$ 0.00$ & 0 & years \\
\hline & & & $x$ & $30 \mathrm{kBtu} / \mathrm{hr}$ & $\$ 88.00$ & 0 & years \\
\hline & & & $x$ & $40 \mathrm{kBtu} / \mathrm{hr}$ & $\$ 117.00$ & 0 & years \\
\hline & & & $x$ & $50 \mathrm{kBtu} / \mathrm{hr}$ & $\$ 146.00$ & 0 & years \\
\hline & & & $x$ & $60 \mathrm{kBtu} / \mathrm{hr}$ & $\$ 175.00$ & 0 & years \\
\hline & & & $x$ & $70 \mathrm{kBtu} / \mathrm{hr}$ & $\$ 204.00$ & 0 & years \\
\hline & & & $x$ & $80 \mathrm{kBtu} / \mathrm{hr}$ & $\$ 234.00$ & 0 & years \\
\hline & & & $x$ & $90 \mathrm{kBtu} / \mathrm{hr}$ & $\$ 263.00$ & 0 & years \\
\hline & & & $x$ & $100 \mathrm{kBtu} / \mathrm{hr}$ & $\$ 292.00$ & 0 & years \\
\hline & & & $x$ & $110 \mathrm{kBtu} / \mathrm{hr}$ & $\$ 321.00$ & 0 & years \\
\hline & & & $x$ & $120 \mathrm{kBtu} / \mathrm{hr}$ & $\$ 350.00$ & 0 & years \\
\hline & & & $x$ & $130 \mathrm{kBtu} / \mathrm{hr}$ & $\$ 380.00$ & 0 & years \\
\hline & & & $x$ & $140 \mathrm{kBtu} / \mathrm{hr}$ & $\$ 409.00$ & 0 & years \\
\hline & & & $x$ & $150 \mathrm{kBtu} / \mathrm{hr}$ & $\$ 438.00$ & 0 & years \\
\hline
\end{tabular}


Appendix B: Example REM/Rate Building File Report Phoenix, Gas Heating 
BUILDING FILE REPORT

File Name: Phoenix Gas.blg

Date: October 28, 2008

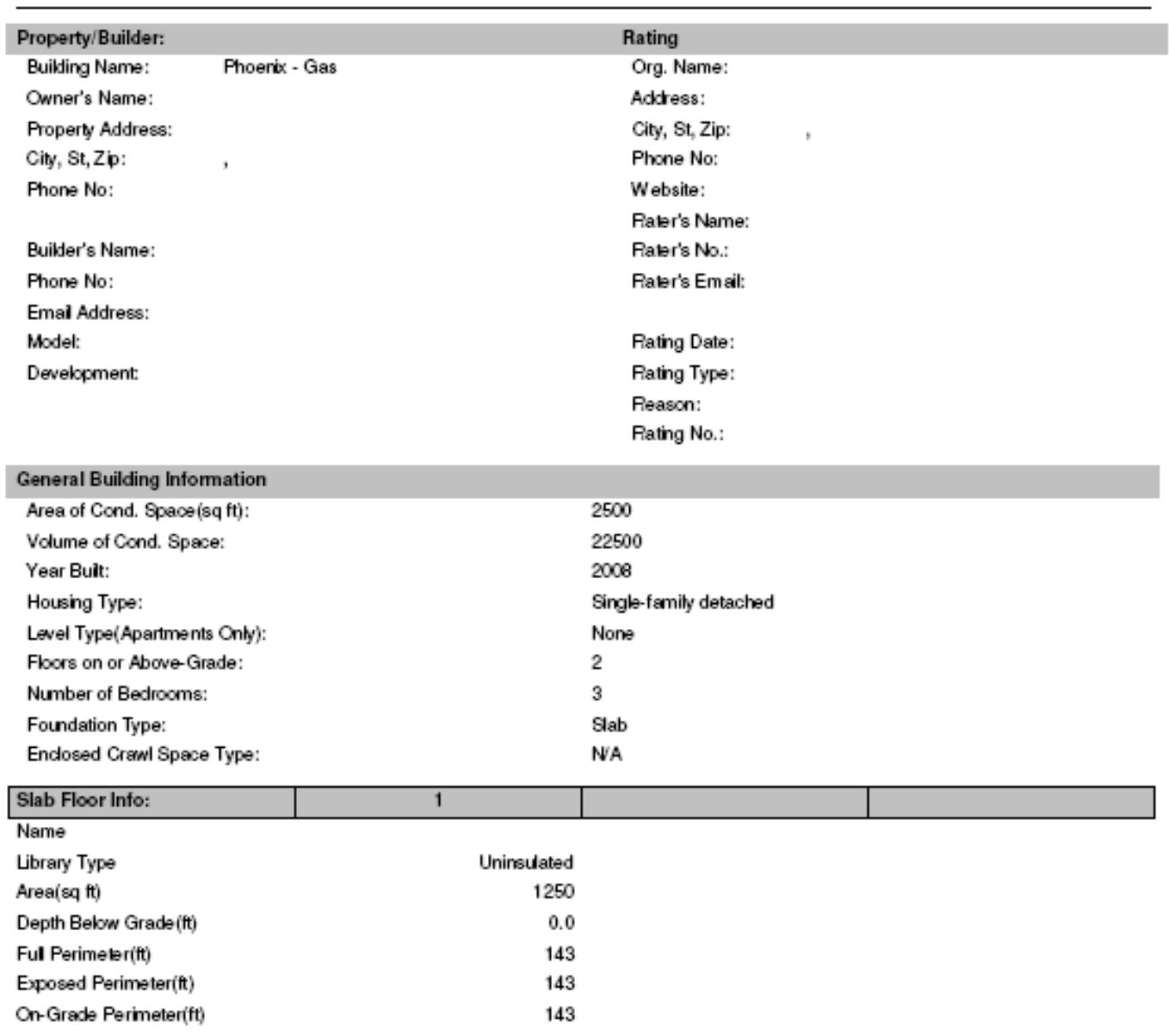

REM Rate - Residential Energy Analysis and Rating Software v12.6

Q 1985-2008 Architectural Energy Corpcrabion, Boulder, Cokrado.

B-2 
BUILDING FILE REPORT

Phoenix - Gas

Page 2

\begin{tabular}{ll}
\hline Slab Floor: Uninsulated & \\
Slab Covering & Carpet \\
Perimeter Insulation (R-Value): & 0.0 \\
Perimeter Insulation Depth (tt): & 0.0 \\
Under-Sab Insulation (R-Value): & 0.0 \\
Under-Sab Insulation Width (ft): & 0.0 \\
Slab Insulation Grade: & 1 \\
Radiant Slab: & No
\end{tabular}

Note:

\begin{tabular}{|l|l|l|l|}
\hline Rim and Band Joist: & 1 & & \\
\hline
\end{tabular}

Name

Area(\&) ft) $\quad 143.0$

Continuous hs $\quad 0.0$

Framed Cavity Ins $\quad 15.0$

Cavity Ins Thk(n) $\quad 5.5$

Joist Spacing 24.0

Location Cond $->$ ambiart

Uo Value $\quad 0.057$

\begin{tabular}{|l|l|l|l|}
\hline Above-Grade Wall: & 1 & & \\
\hline
\end{tabular}

Name

Library Type

R-15, Grade I

2574.00

Gross Area(sq fit)

Madium

Exterior Color

Location

Cond $>$ ambiark

Uo Value

0.079

REW Rate - Residential Energy Analysis and Rating Software v12.6

Q 1985-2008 Architectural Energy Corpcrabion, Boulder, Cokrada

B-3 


\section{BUILDING FILE REPORT}

Phoenix - Gas

Page 3

Above-Grade Wall: R-15, Grade I

Information From Quick Fll Screen:

Standard Wood Frame

Continuous hsulation (R-Value)

Frame Cavity Insulation (R-Value)

Frame Cavity Insulation Thickness (in)

Frame Cavity Insulation Grade

Stud Size ( $w \times d$, in)

Stud Spacing (in o.c.)

Framing Factor - (default)

Gypeum Thickness (in)

Note:

Layers

Inside Air Fim

Gyp board

Air GapiFrm

Cavity ins/Frm

Continuous ins

Ext Finish

Outside Air Film

Total R-Value

U-Value

Relative Area

UA

$\begin{array}{rrrr}\text { Paths } & & & \\ \text { Cavity } & & \text { Framing } & \text { Grade } \\ & 0.680 & 0.680 & 0.680 \\ 0.450 & 0.450 & 0.450 \\ 0.000 & 0.000 & 0.000 \\ 15.000 & 4.375 & 1.030 \\ 0.000 & 0.000 & 0.000 \\ 0.940 & 0.940 & 0.940 \\ 0.000 & 0.000 & 0.000 \\ & & \\ 0.170 & 0.170 & 0.170 \\ & & \\ 17.240 & 6.615 & 3.270 \\ 0.058 & 0.151 & 0.306 \\ & & \\ 0.770 & 0.230 & 0.000 \\ 0.045 & 0.035 & 0.000\end{array}$

Total Component UA: 0.079

Total Component Area: 1.0

Component Ub: 0.079 
BUILDING FILE REPORT

Phoenix - Gas

Page 4

\begin{tabular}{|c|c|c|c|c|}
\hline Window Information: & 1 & & 2 & 3 \\
\hline Name & North & & South & West \\
\hline Library Type & U 0.35 ; SHGC 0.35 & & U $0.35 ;$ SHGC 0.35 & U 0.36 ; SHGC 0.35 \\
\hline U-value & 0.350 & & 0.350 & 0.350 \\
\hline $\mathrm{SHGC}$ & 0.350 & & 0.350 & 0.350 \\
\hline Area $(\& q \mathrm{ft})$ & 112.50 & & 112.50 & 11250 \\
\hline Orientation & North & & South & West \\
\hline Overhang Depth & 0.0 & & 0.0 & 0.0 \\
\hline Overhang To Top & 0.0 & & 0.0 & 0.0 \\
\hline Overhang To Bottom & 0.0 & & 0.0 & 0.0 \\
\hline Interior Winter Shading & 0.85 & & 0.85 & 0.85 \\
\hline Intericr Oummer Shading & 0.70 & & 0.70 & 0.70 \\
\hline Adjacent Winter Shading & None & & None & None \\
\hline Adjacent Summer Shading & None & & None & None \\
\hline Wall Assignment & AGW all 1 & & AGWall 1 & AGWall 1 \\
\hline Window Information: & 4 & & & \\
\hline Name & East & & & \\
\hline Library Type & U $0.35 ;$ SHGC 0.35 & & & \\
\hline u-value & 0.350 & & & \\
\hline$S H G C$ & 0.350 & & & \\
\hline Area( $(q \mathrm{ft})$ & 112.50 & & & \\
\hline Crientation & East & & & \\
\hline Overhang Depth & 0.0 & & & \\
\hline Overhang To Top & 0.0 & & & \\
\hline Overhang To Bottom & 0.0 & & & \\
\hline Interior Winter Shading & 0.85 & & & \\
\hline Intericr Summer Shading & 0.70 & & & \\
\hline Adjacent Winter Shading & None & & & \\
\hline Adjacent Summer Shading & None & & & \\
\hline Wall Assignment & AGW all 1 & & & \\
\hline \multicolumn{5}{|l|}{ Window: U 0.35 ; SHGC 0.35} \\
\hline U-Value: & & 0.350 & & \\
\hline Solar Heat Gain Coefficient: & & 0.350 & & \\
\hline \multicolumn{5}{|l|}{ Note: } \\
\hline Door Information: & 1 & & & \\
\hline \multicolumn{5}{|l|}{ Name } \\
\hline Opaque Area(sq ft) & 40.0 & & & \\
\hline Library Type & $2-1 / 4$ Wd solid, strm & & & \\
\hline Wall Assignment & AGW all 1 & & & \\
\hline Uo Valus & 0.211 & & & \\
\hline
\end{tabular}

REM Rate - Residential Energy Analysis and Rating Software v12.6

Q 1985-2008 Architectural Energy Corpcrabion, Boulder, Cokraja. 
BUILDING FILE REPORT

Phoenix - Gas

Page 5

Door: 2-1/4 W/d solid, strm

R-Value of Opeque Area:

Storm Door:

2.8

Note:

Roof Information:

Name

Library Type

Gross Area(sq ft)

Color

Radiant Barrier

Type(Attic)

Uo Value

R-30 Blown, Attic
1250.00
Light
No
Attic
0.034

REM Rate - Residential Energy Analysis and Rating Software v12.6

9 1985-2008 Architectural Energy Corpcrabion, Boubler, Cokcrado. 


\begin{tabular}{|c|c|}
\hline Phoenix - Gas & Page 6 \\
\hline \multicolumn{2}{|l|}{ Ceiling: R-30 Blown, Attic } \\
\hline \multicolumn{2}{|l|}{ Information From Cuick Fil Screen: } \\
\hline Conthous Insulation (R-Valus) & 17.0 \\
\hline Cavity Insulation (R-Value) & 13.0 \\
\hline Cavity Irsulation Thickness (in) & 3.5 \\
\hline Cavity Insulation Grads & 3.0 \\
\hline Gypsum Thickness (n) & 0.500 \\
\hline Bottom Chord/Rafter Size $(w \times h, \mathbf{n})$ & $1.5 \times 3.5$ \\
\hline Bottom Chord/Rafter Spachy (in o.c.) & 24.0 \\
\hline Framing Factor - (default) & 0.1100 \\
\hline Celing Type & Attic \\
\hline
\end{tabular}

Note:

\begin{tabular}{|c|c|c|c|}
\hline \multirow[t]{2}{*}{ Layers } & \multicolumn{3}{|l|}{ Paths } \\
\hline & Framing & Cavity & Grada \\
\hline Inside Air Fim & 0.610 & 0.610 & 0.610 \\
\hline Gyp board & 0.450 & 0.450 & 0.450 \\
\hline Cavity Ins/Frm & 4.375 & 13.000 & 0.000 \\
\hline \multirow[t]{4}{*}{ Continuous ins } & 17.000 & 17.000 & 17.000 \\
\hline & 0.000 & 0.000 & 0.000 \\
\hline & 0.000 & 0.000 & 0.000 \\
\hline & 0.000 & 0.000 & 0.000 \\
\hline Cutside Air Film & 0.610 & 0.610 & 0.610 \\
\hline Total R-Valus & 23.045 & 31.670 & 18.670 \\
\hline U-Value & 0.043 & 0.032 & 0.054 \\
\hline Relative Area & 0.110 & 0.840 & 0.050 \\
\hline UA & 0.005 & 0.027 & 0.003 \\
\hline
\end{tabular}

Total Component UA: 0.034

Total Component Area: 1.0

Component Ub: 0.034

\section{Mechanical Equipment: General}

Number of Machanical Systerns:

Heating SetPoint(F):

3

Heating Setback Thermostat:

Cooling SetPoint $F$ ):

68.00

Present

Cooling Setup Thermostat:

78.00

Present

REIW Rate - Residential Energy Analysis and Rating Software v12. 6

Q 1985-2008 Architectural Energy Corpcration, Boulder, Cckorada. 
BUILDING FILE REPORT

\begin{tabular}{|c|c|c|}
\hline \multicolumn{2}{|l|}{ Phoenix - Gas } & \multirow[t]{2}{*}{ Page 7} \\
\hline Heat: 82AFUE Gas Furn 80k & & \\
\hline SystemType: & Fualfired air distrbution & \\
\hline Fuel Type: & Natural gas & \\
\hline Rated Output Capacity (kBtuh): & 80.0 & \\
\hline Seasona Equipment Efficiency: & 82.0 AFUE & \\
\hline Auxiliary Electric: & 910 Eae & \\
\hline Note: & & \\
\hline Location: & Attic & \\
\hline Performance Adjustment: & 100 & \\
\hline Percent Load Served: & 100 & \\
\hline Number Of Units: & 1 & \\
\hline \multicolumn{3}{|c|}{ Cooling Equipment: 14SEER A/C 2 ton } \\
\hline System Type: & Air conditioner & \\
\hline Fuel Type: & Electric & \\
\hline Rated Output Capacity (kBtuh): & 24.0 & \\
\hline Seasonal Equipment Efficiency: & 14.0 SEER & \\
\hline Sensble Heat Fraction (SHF): & 0.70 & \\
\hline \multicolumn{3}{|l|}{ Note: } \\
\hline Location: & Attic & \\
\hline Performance Adjustment: & 100 & \\
\hline Percent Loed Served: & 100 & \\
\hline Number Of Units: & 1 & \\
\hline \multicolumn{3}{|c|}{ Water Heating Equipment: 40 gal. $0.59 E F$ Gas } \\
\hline Water He ater Type: & Comentional & \\
\hline Fuel Type: & Natural gas & \\
\hline Energy Factor: & 0.59 & \\
\hline Recovery Efficiency: & 0.76 & \\
\hline Water Tank Size (gallons): & 40 & \\
\hline Extra Tank hsulation (R-Value): & 0.0 & \\
\hline \multicolumn{3}{|l|}{ Note: } \\
\hline Location: & Attic & \\
\hline Percent Loed Served: & 100 & \\
\hline Performance Adjustment: & 100 & \\
\hline Number Of Units: & 1 & \\
\hline
\end{tabular}

REM Rate - Residential Energy Analysis and Rating Software v12.6

Q 1985-2008 Architectural Energy Corpcrabon, Boulder, Cokrasa. 
BUILDING FILE REPORT

Phoenix - Gas

Page 8

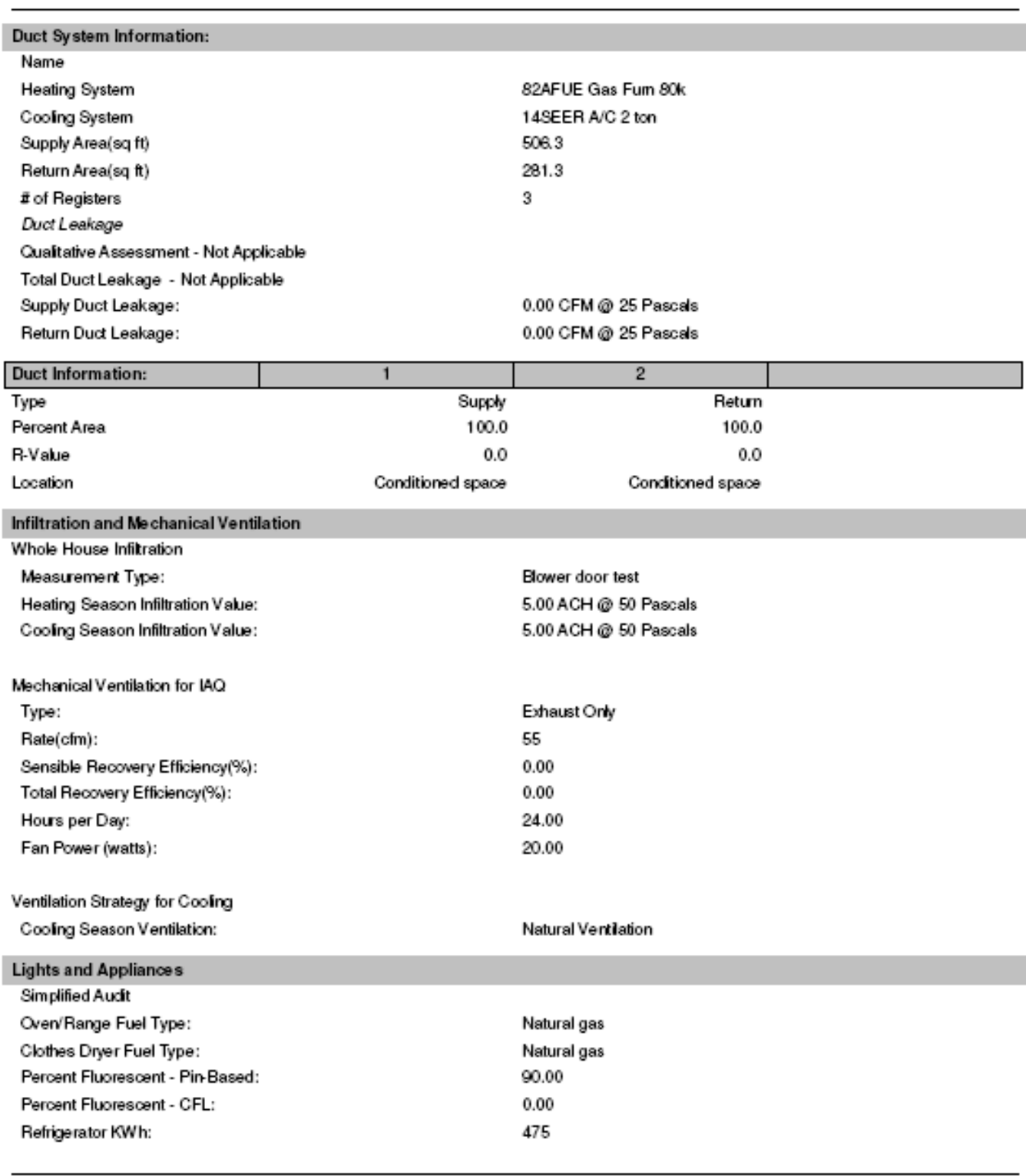

REM Rate - Residential Energy Analysis and Rating Software v12.6

Q 1985-2008 Architectural Energy Corpcrabon, Boulder, Cokrada. 


\section{BUILDING FILE REPORT}

Phoenix - Gas

Page 9

Lights and Appliances

Dishwasher EF:

Celing Fan CFM / Watt:

0.75

0.00

REM Rate - Residential Energy Analysis and Rating Software v12.6

Q 1985-2008 Architectural Energy Corpcration, Boulder, Cokrasa. 
Appendix C: Example EnergyGauge Building Input Report Phoenix, Gas Heating 


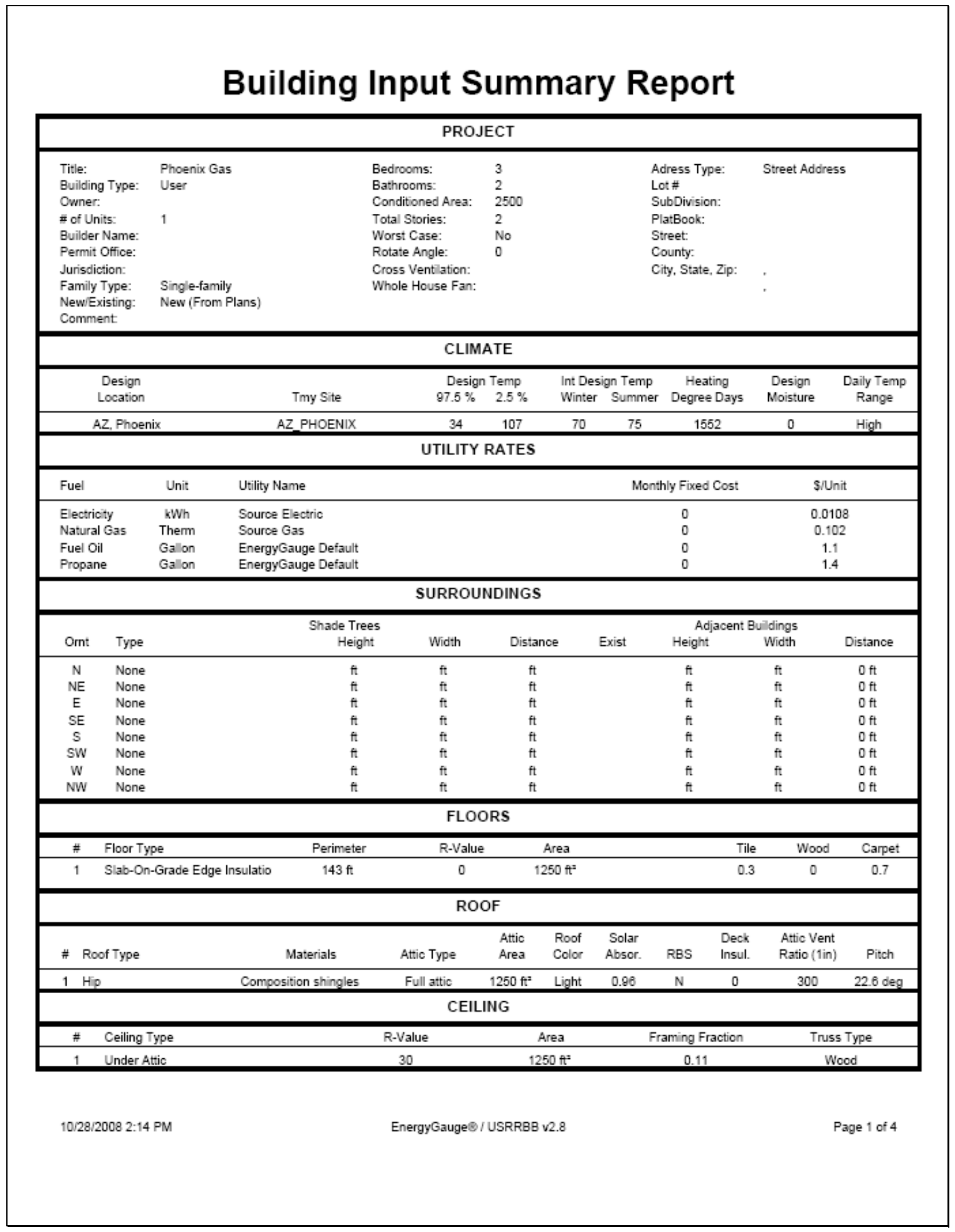




\section{Building Input Summary Report}

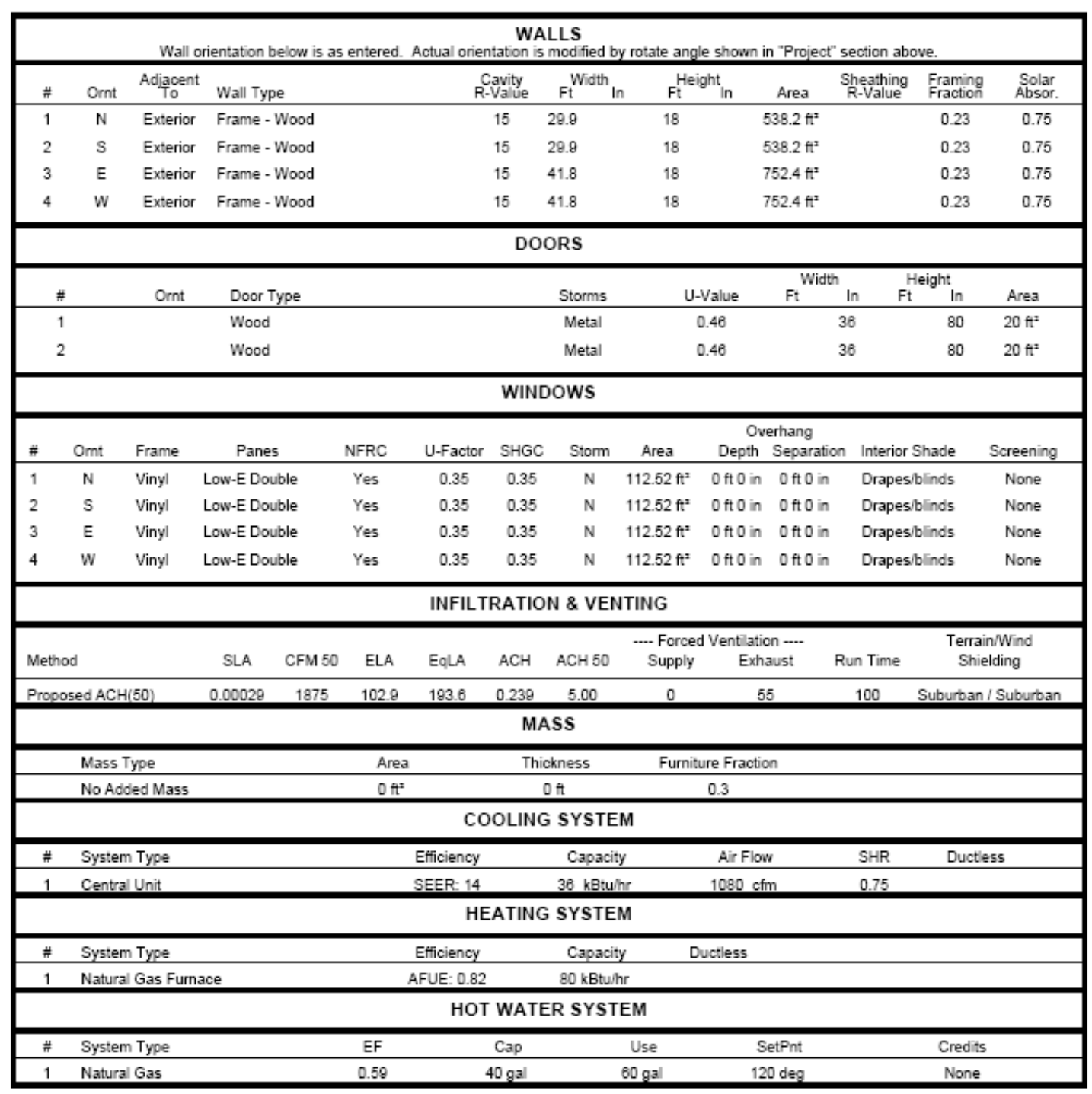




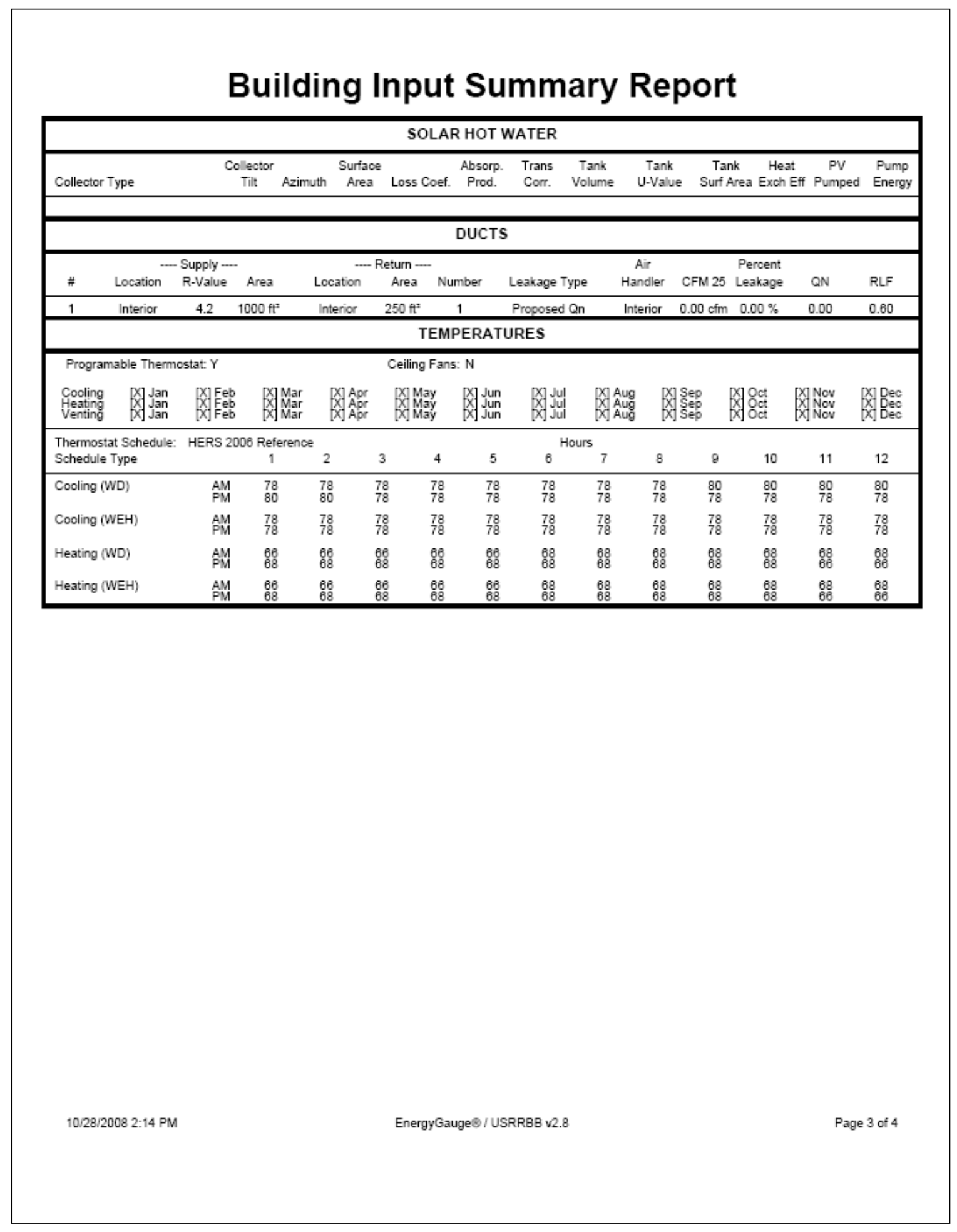




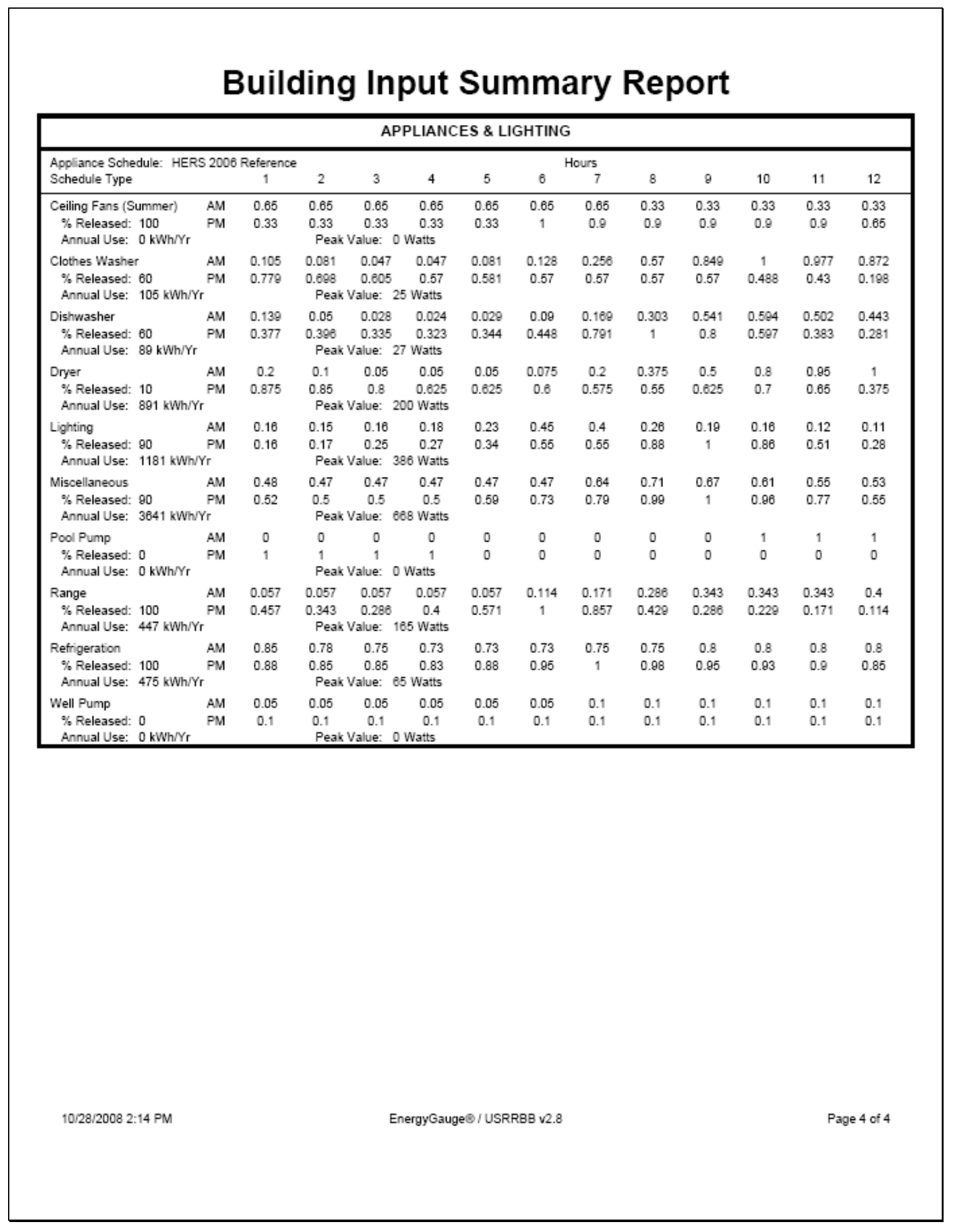


Appendix D: BEopt Results 


\section{Figures}

Figure D-1. Minimum-cost point for Atlanta with gas space- and water-heating................................ D-3

Figure D-2. Near-neutral-cost point for Atlanta with gas space- and water-heating............................ D-4

Figure D-3. Minimum-cost point for Atlanta with electric space- and water-heating. ........................... D-5

Figure D-4. Near-neutral-cost point for Atlanta with electric space- and water-heating. ..................... D-6

Figure D-5. Minimum-cost point for Chicago with gas space- and water-heating. ............................... D-7

Figure D-6. Near-neutral-cost point for Chicago with gas space- and water-heating. ........................... D-8

Figure D-7. Minimum-cost point for Chicago with electric space- and water-heating. ......................... D-9

Figure D-8. Near-neutral-cost point for Chicago with electric space- and water-heating. ................... D-10

Figure D-9. Minimum-cost point for Houston with gas space- and water-heating.............................. D-11

Figure D-10. Near-neutral-cost point for Houston with gas space- and water-heating...................... D-12

Figure D-11. Minimum-cost point for Houston with electric space- and water-heating. ..................... D-13

Figure D-12. Near neutral-cost point for Houston with electric space- and water-heating.................. D-14

Figure D-13. Minimum-cost point for Phoenix with gas space- and water-heating............................ D-15

Figure D-14. Near-neutral-cost point for Phoenix with gas space- and water-heating....................... D-16

Figure D-15. Minimum-cost point for Phoenix with electric space- and water-heating. ..................... D-17

Figure D-16. Near-neutral-cost point for Phoenix with electric space- and water-heating. ................. D-18

Figure D-17. Minimum-cost point for Seattle with gas space- and water-heating. ............................ D-19

Figure D-18. Near-neutral-cost point for Seattle with gas space- and water-heating. ........................ D-20

Figure D-19. Minimum-cost point for Seattle with electric space- and water-heating........................ D-21

Figure D-20. Near-neutral-cost point for Seattle with electric space- and water-heating.................... D-22 
Minimum-Cost / Atlanta / Gas Heat

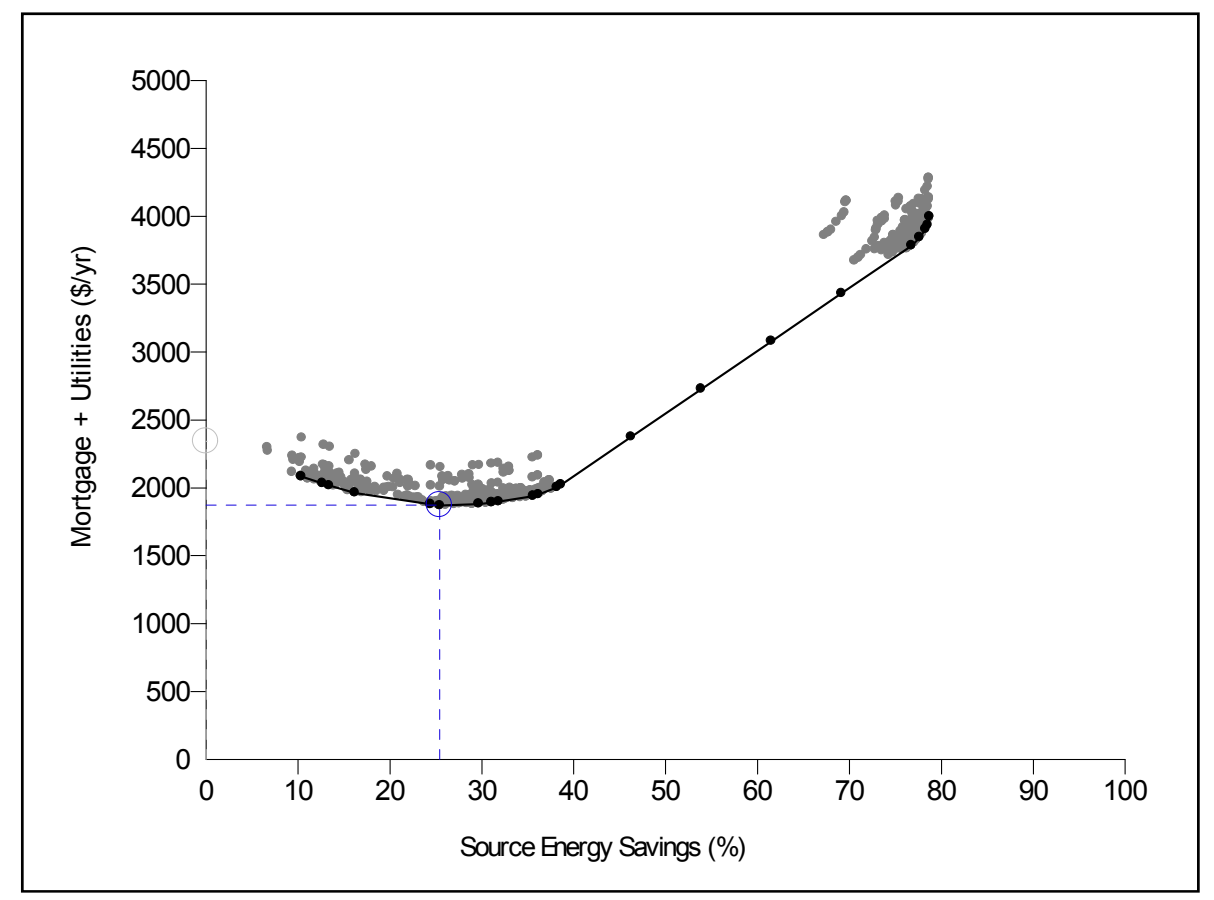

\begin{tabular}{|l|l|r|}
\hline Component & Efficiency Level & Incremental Cost \\
\hline Wall Insulation & R15 & $\$ 87$ \\
\hline Ceiling Insulation & R30 & $\$ 0$ \\
\hline Radiant Barrier & None & $\$ 0$ \\
\hline Infiltration & 0.0003 SLA & $\$ 1,821$ \\
\hline Window Type & Low-e low SHGC arg & $\$ 0$ \\
\hline Slab & No Slab & $\$ 0$ \\
\hline Basement & No Basement & $\$ 0$ \\
\hline Crawl Space & Unvented R10 Interior & $(\$ 819)$ \\
\hline Hardwired Lighting & $100 \%$ Fluorescent & $\$ 194$ \\
\hline Air Conditioner & SEER 13 & $\$ 0$ \\
\hline Furnace & AFUE 92.5\% & $\$ 348$ \\
\hline Heat Pump & No Heat Pump & $\$ 0$ \\
\hline Water Heater & Gas Tankless & $\$ 625$ \\
\hline Ducts & R-4.2, 10\% leakage & $\$ 0$ \\
\hline Solar DHW & No Solar DHW & $\$ 0$ \\
\hline PV Size & 0 kW & $\$ 0$ \\
\hline Cooling Capacity Reduction & 0.5 tons & $(\$ 272)$ \\
\hline Heating Capacity Reduction & 20 kBtu/hr & $(\$ 70)$ \\
\hline Total & & $\$ 1,914$ \\
\hline
\end{tabular}

Figure D-1. Minimum-cost point for Atlanta with gas space- and water-heating. 
Neutral-Cost / Atlanta / Gas Heat

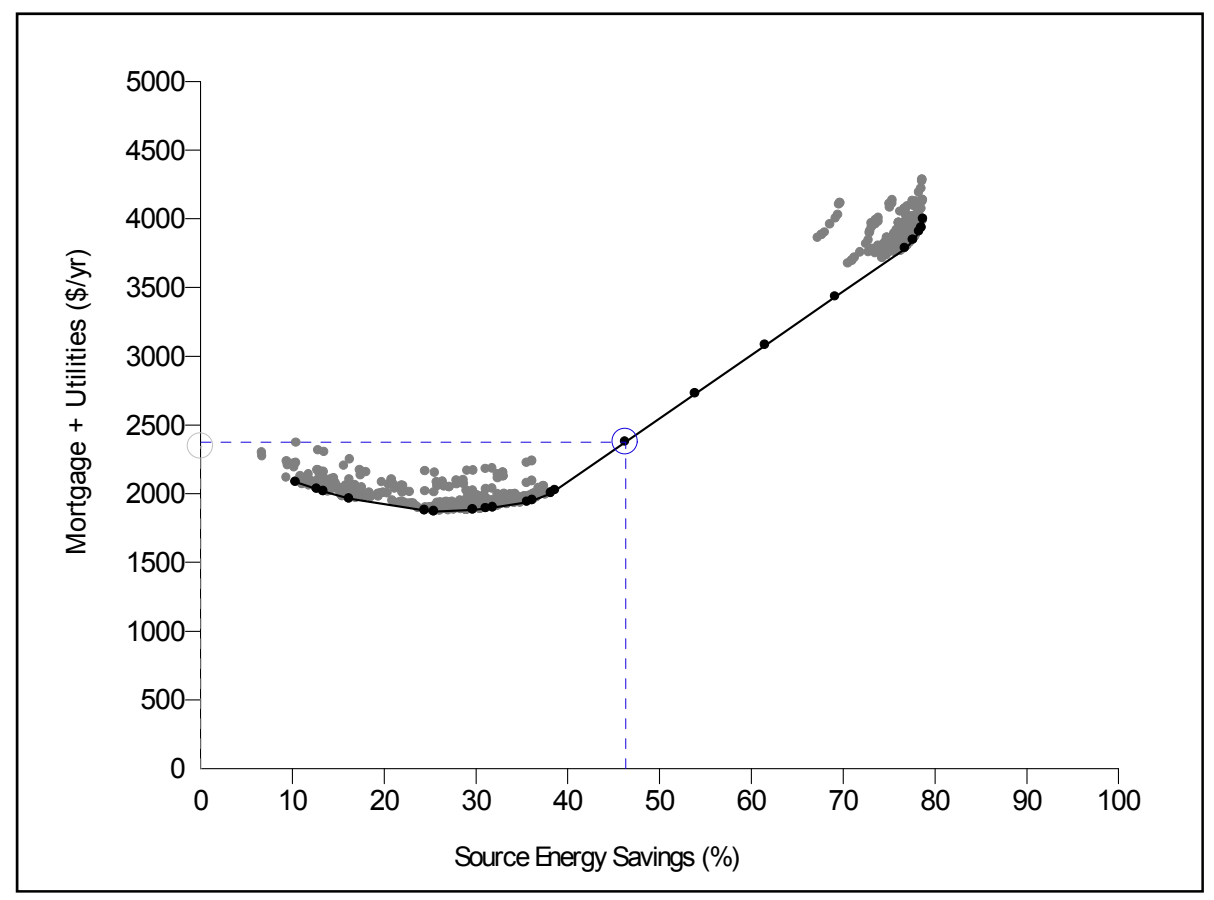

\begin{tabular}{|l|l|r|}
\hline Component & Efficiency Level & Incremental Cost \\
\hline Wall Insulation & R15 & $\$ 87$ \\
\hline Ceiling Insulation & R40 & $\$ 472$ \\
\hline Radiant Barrier & Radiant Barrier & $\$ 483$ \\
\hline Infiltration & 0.00015 SLA & $\$ 3,642$ \\
\hline Window Type & Low-e low SHGC arg & $\$ 0$ \\
\hline Slab & No Slab & $\$ 0$ \\
\hline Basement & No Basement & $\$ 0$ \\
\hline Crawl Space & Unvented R10 Interior & $(\$ 819)$ \\
\hline Hardwired Lighting & $100 \%$ Fluorescent & $\$ 194$ \\
\hline Air Conditioner & SEER 17 & $\$ 720$ \\
\hline Furnace & AFUE 92.5\% & $\$ 348$ \\
\hline Heat Pump & No Heat Pump & $\$ 0$ \\
\hline Water Heater & Gas Tankless & $\$ 625$ \\
\hline Ducts & R-4.2, 10\% leakage & $\$ 0$ \\
\hline Solar DHW & 64 sq ft closed loop & $\$ 4,768$ \\
\hline PV Size & $1.0 \mathrm{~kW}$ & $\$ 7,500$ \\
\hline Cooling Capacity Reduction & 1 tons & $(\$ 545)$ \\
\hline Heating Capacity Reduction & 30 kBtu/hr & $(\$ 104)$ \\
\hline Total & & $\$ 17,371$ \\
\hline
\end{tabular}

Figure D-2. Near-neutral-cost point for Atlanta with gas space- and water-heating. 


\section{Minimum-Cost / Atlanta / Electric Heat}

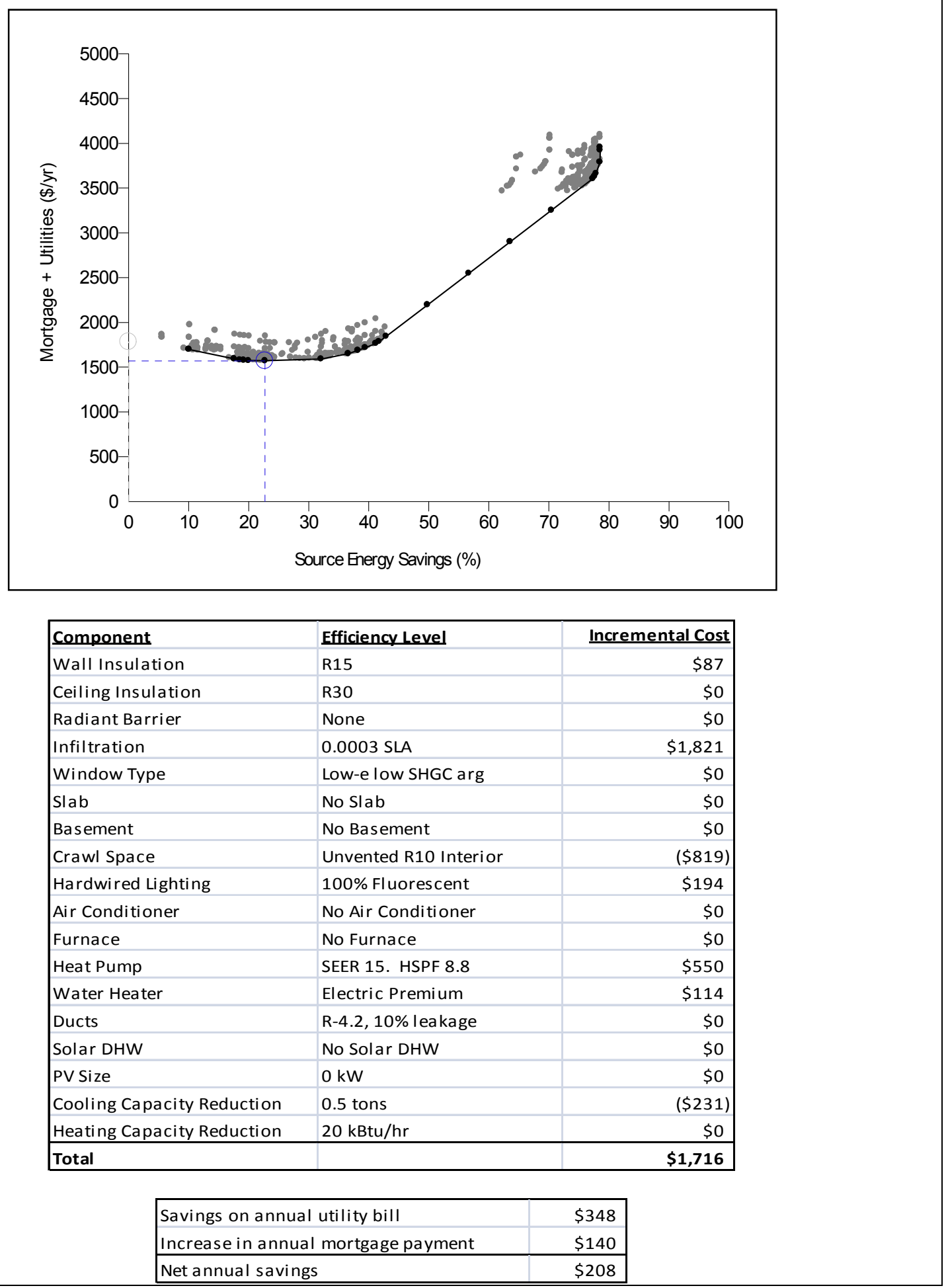

Figure D-3. Minimum-cost point for Atlanta with electric space- and water-heating. 


\section{Neutral-Cost / Atlanta / Electric Heat}

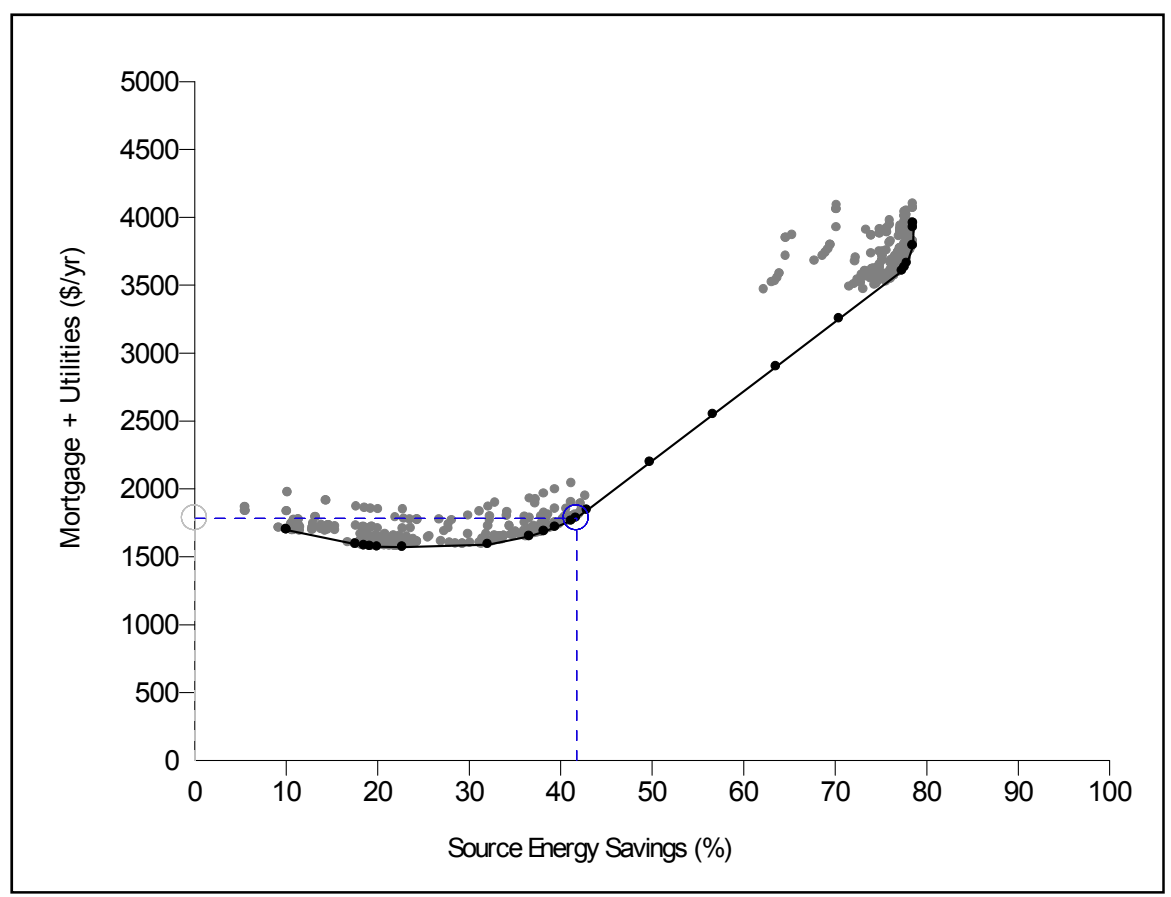

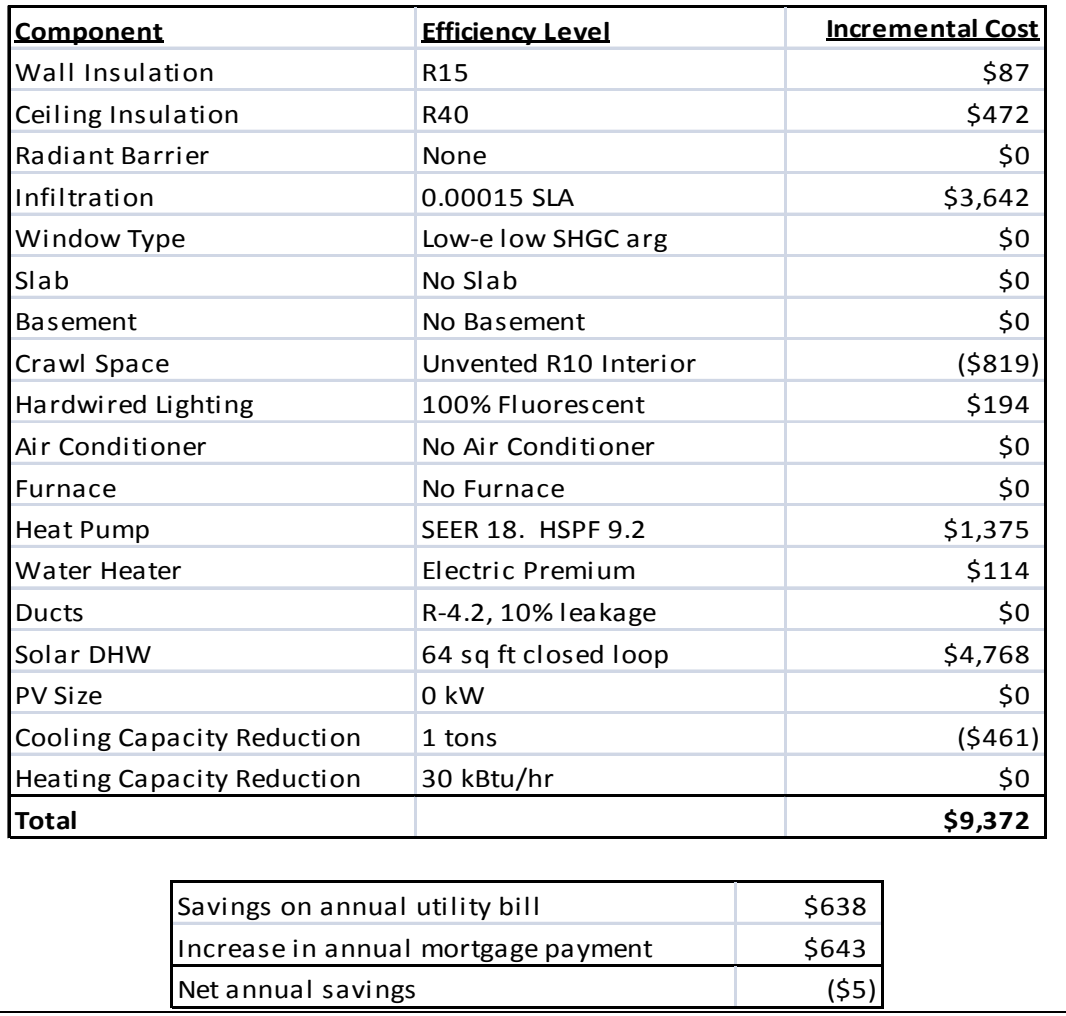

Figure D-4. Near-neutral-cost point for Atlanta with electric space- and water-heating. 


\section{Minimum-Cost / Chicago / Gas Heat}

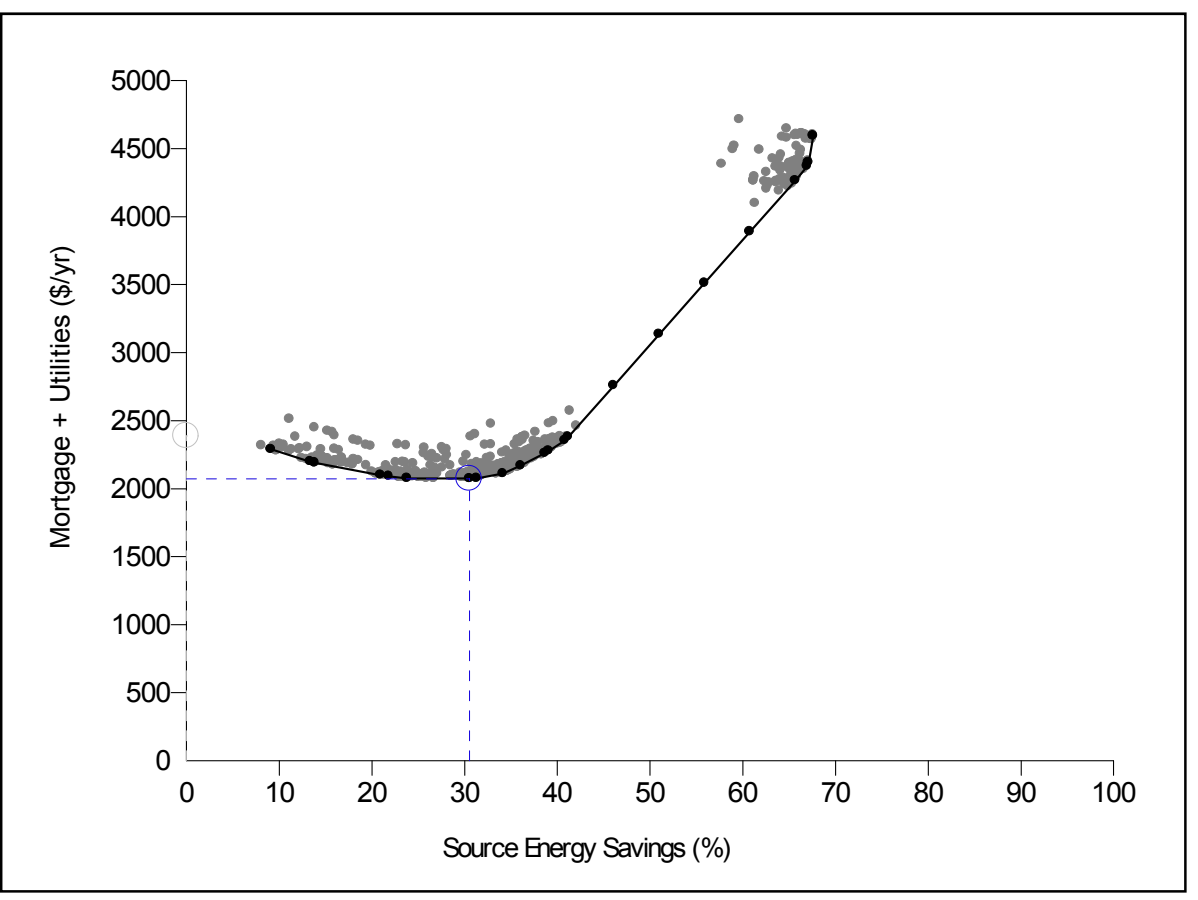

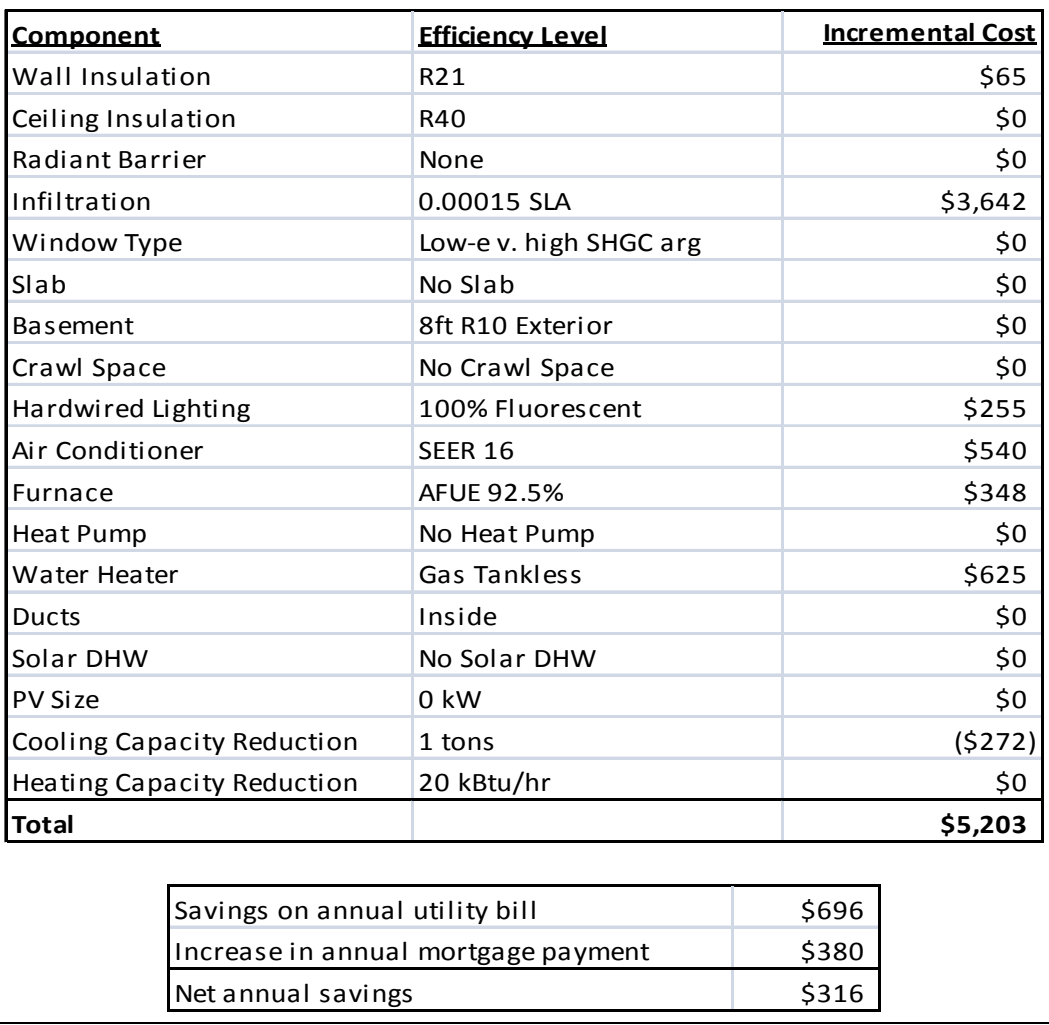

Figure D-5. Minimum-cost point for Chicago with gas space- and water-heating. 


\section{Neutral-Cost / Chicago / Gas Heat}

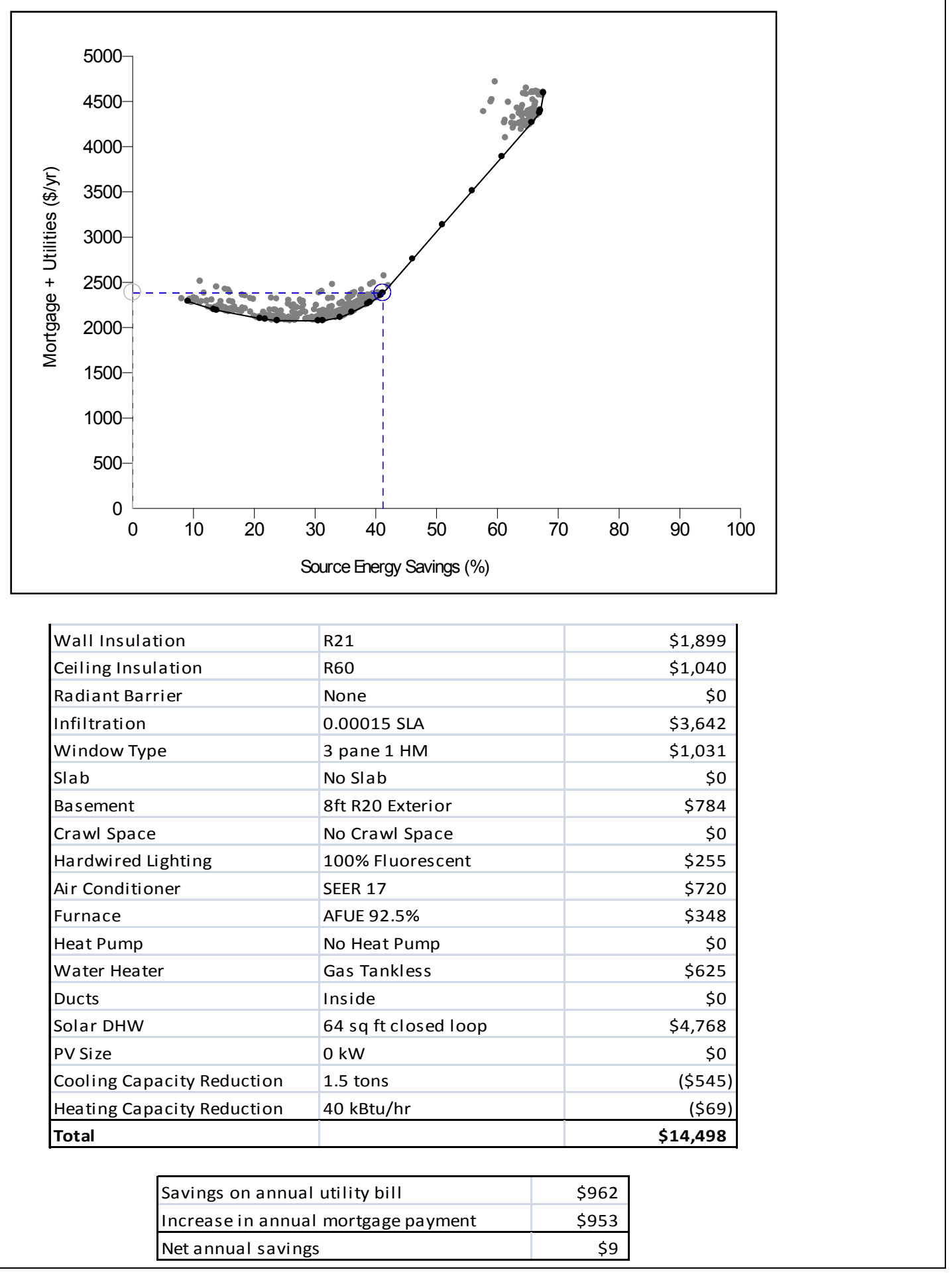

Figure D-6. Near-neutral-cost point for Chicago with gas space- and water-heating. 


\section{Minimum-Cost / Chicago / Electric Heat}

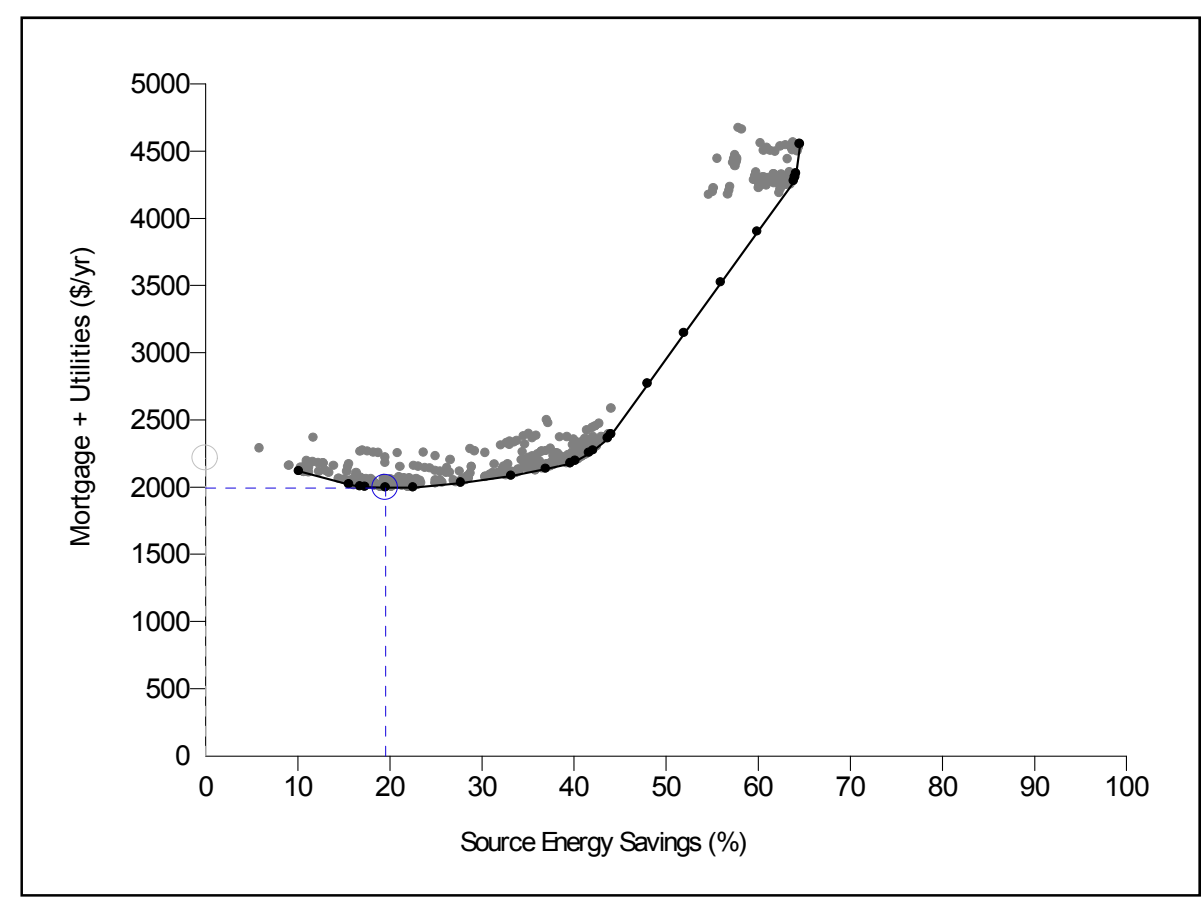

\begin{tabular}{|c|c|c|c|}
\hline Component & Efficiency Level & $\underline{\text { Incr }}$ & ntal Cost \\
\hline Wall Insulation & R21 & & $\$ 65$ \\
\hline Ceiling Insulation & R40 & & $\$ 0$ \\
\hline Radiant Barrier & None & & $\$ 0$ \\
\hline Infiltration & 0.0003 SLA & & $\$ 1,821$ \\
\hline Window Type & Low-e v. high SHGC arg & & $\$ 0$ \\
\hline Slab & No Slab & & $\$ 0$ \\
\hline Basement & $8 \mathrm{ft}$ R10 Exterior & & $\$ 0$ \\
\hline Crawl Space & No Crawl Space & & $\$ 0$ \\
\hline Hardwired Lighting & 100\% Fluorescent & & $\$ 255$ \\
\hline Air Conditioner & No Air Conditioner & & $\$ 0$ \\
\hline Furnace & No Furnace & & $\$ 0$ \\
\hline Heat Pump & SEER 15. HSPF 8.8 & & $\$ 550$ \\
\hline Water Heater & Electric Premium & & $\$ 114$ \\
\hline Ducts & Inside & & $\$ 0$ \\
\hline Solar DHW & No Solar DHW & & $\$ 0$ \\
\hline PV Size & $0 \mathrm{~kW}$ & & $\$ 0$ \\
\hline Cooling Capacity Reduction & 1 tons & & $(\$ 461)$ \\
\hline Heating Capacity Reduction & $10 \mathrm{kBtu} / \mathrm{hr}$ & & $\$ 0$ \\
\hline Total & & & $\$ 2,344$ \\
\hline Savings on ann & utility bill & $\$ 397$ & \\
\hline Increase in ann & mortgage payment & $\$ 175$ & \\
\hline Net annual savi & & $\$ 222$ & \\
\hline
\end{tabular}

Figure D-7. Minimum-cost point for Chicago with electric space- and water-heating. 


\section{Neutral-Cost / Chicago / Electric Heat}

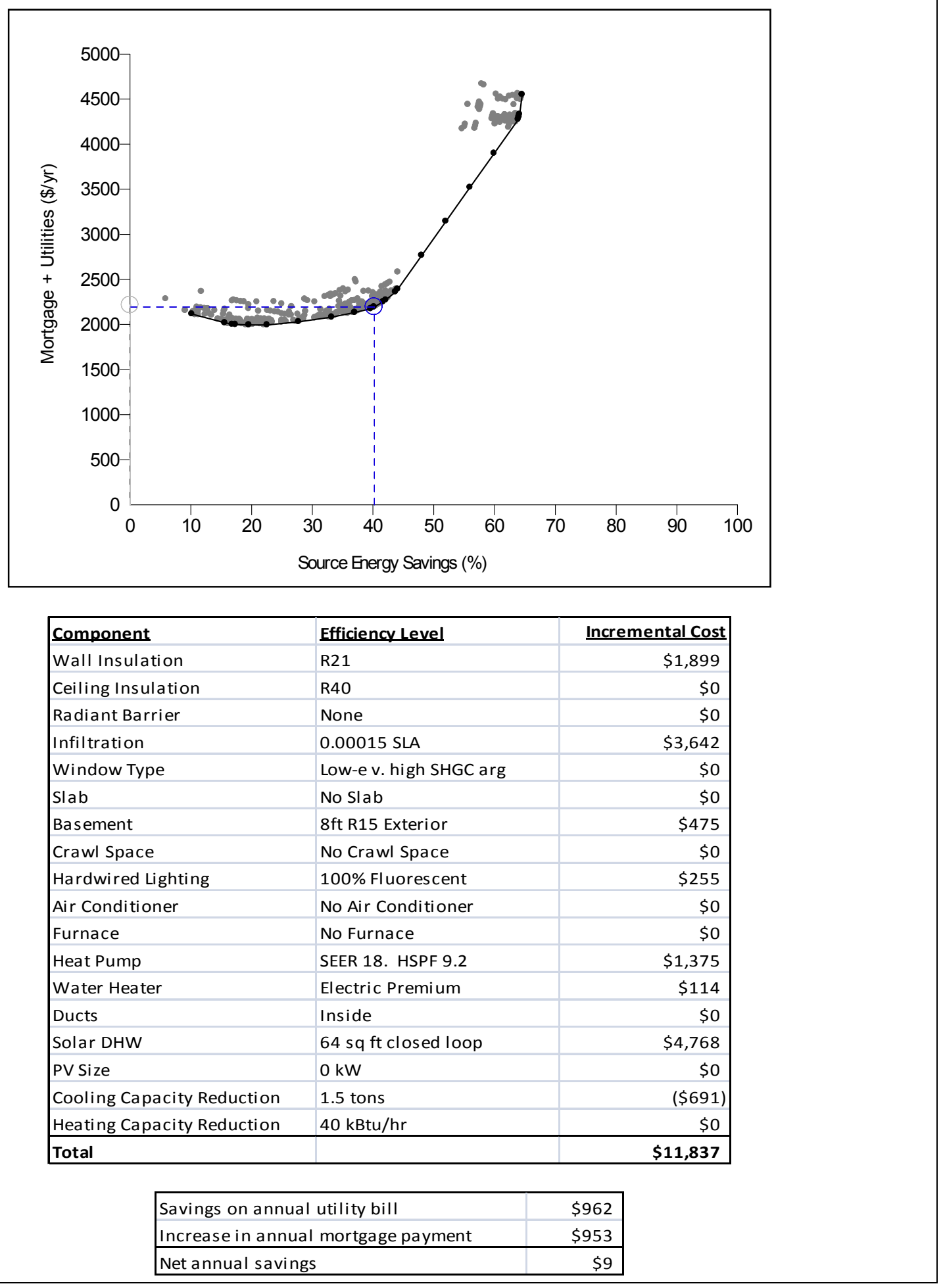

Figure D-8. Near-neutral-cost point for Chicago with electric space- and water-heating. 


\section{Minimum-Cost / Houston / Gas Heat}

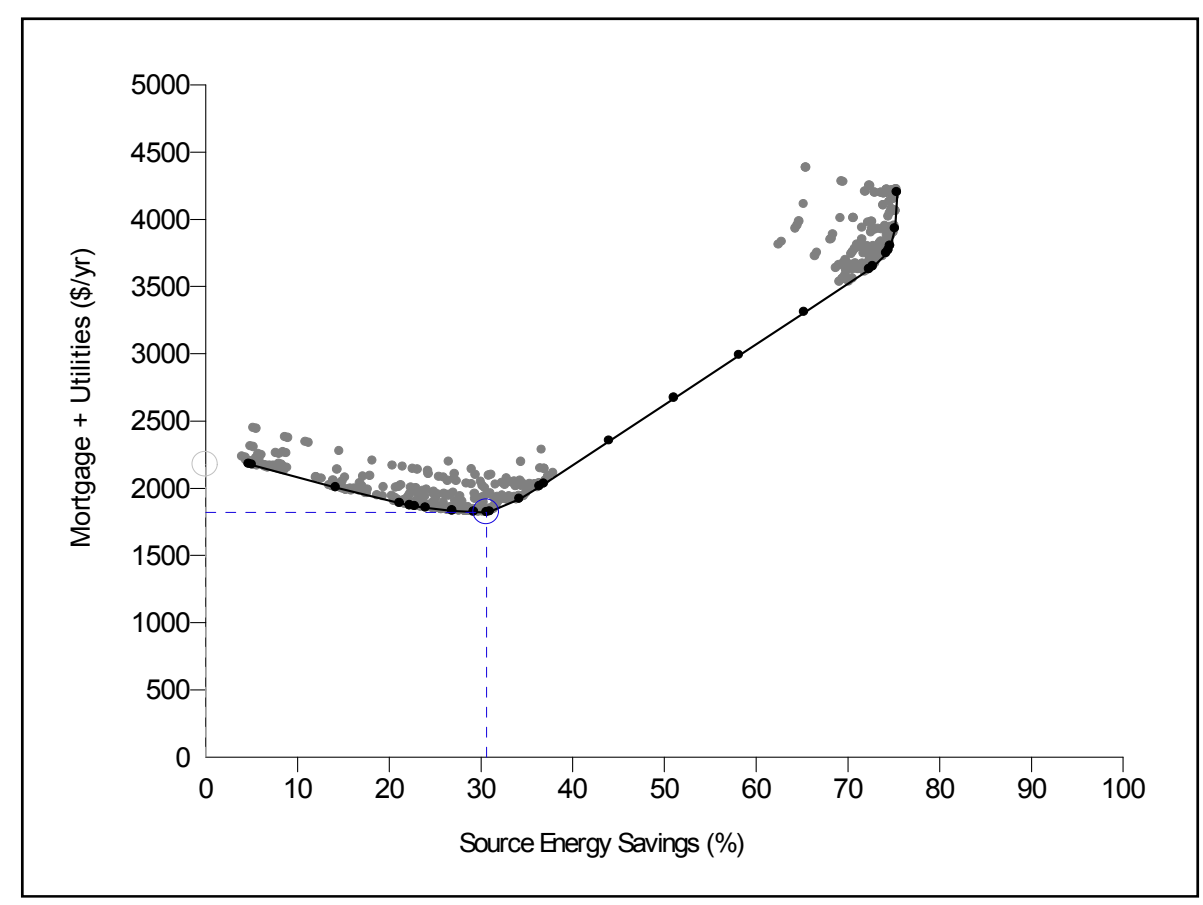

\begin{tabular}{|c|c|c|c|}
\hline Component & Efficiency Level & $\underline{\text { Incr }}$ & ntal Cost \\
\hline Wall Insulation & R15 & & $\$ 87$ \\
\hline Ceiling Insulation & R30 & & $\$ 0$ \\
\hline Radiant Barrier & None & & $\$ 0$ \\
\hline Infiltration & 0.0003 SLA & & $\$ 1,821$ \\
\hline Window Type & Low-e low SHGC arg & & $\$ 0$ \\
\hline Slab & Uninsulated & & $\$ 0$ \\
\hline Basement & No Basement & & $\$ 0$ \\
\hline Crawl Space & No Crawl Space & & $\$ 0$ \\
\hline Hardwired Lighting & 100\% Fluorescent & & $\$ 194$ \\
\hline Air Conditioner & SEER 17 & & $\$ 720$ \\
\hline Furnace & AFUE $92.5 \%$ & & $\$ 348$ \\
\hline Heat Pump & No Heat Pump & & $\$ 0$ \\
\hline Water Heater & Gas Tankless & & $\$ 625$ \\
\hline Ducts & Inside & & $\$ 947$ \\
\hline Solar DHW & No Solar DHW & & $\$ 0$ \\
\hline PV Size & $0 \mathrm{~kW}$ & & $\$ 0$ \\
\hline Cooling Capacity Reduction & 2.5 tons & & $(\$ 1,362)$ \\
\hline Heating Capacity Reduction & $-40 \mathrm{kBtu} / \mathrm{hr}$ & & $\$ 139$ \\
\hline Total & & & $\$ 3,519$ \\
\hline Savings on ann & utility bill & $\$ 608$ & \\
\hline Increase in ann & mortgage payment & $\$ 250$ & \\
\hline Net annual savi & & $\$ 358$ & \\
\hline
\end{tabular}

Figure D-9. Minimum-cost point for Houston with gas space- and water-heating. 


\section{Neutral-Cost / Houston / Gas Heat}

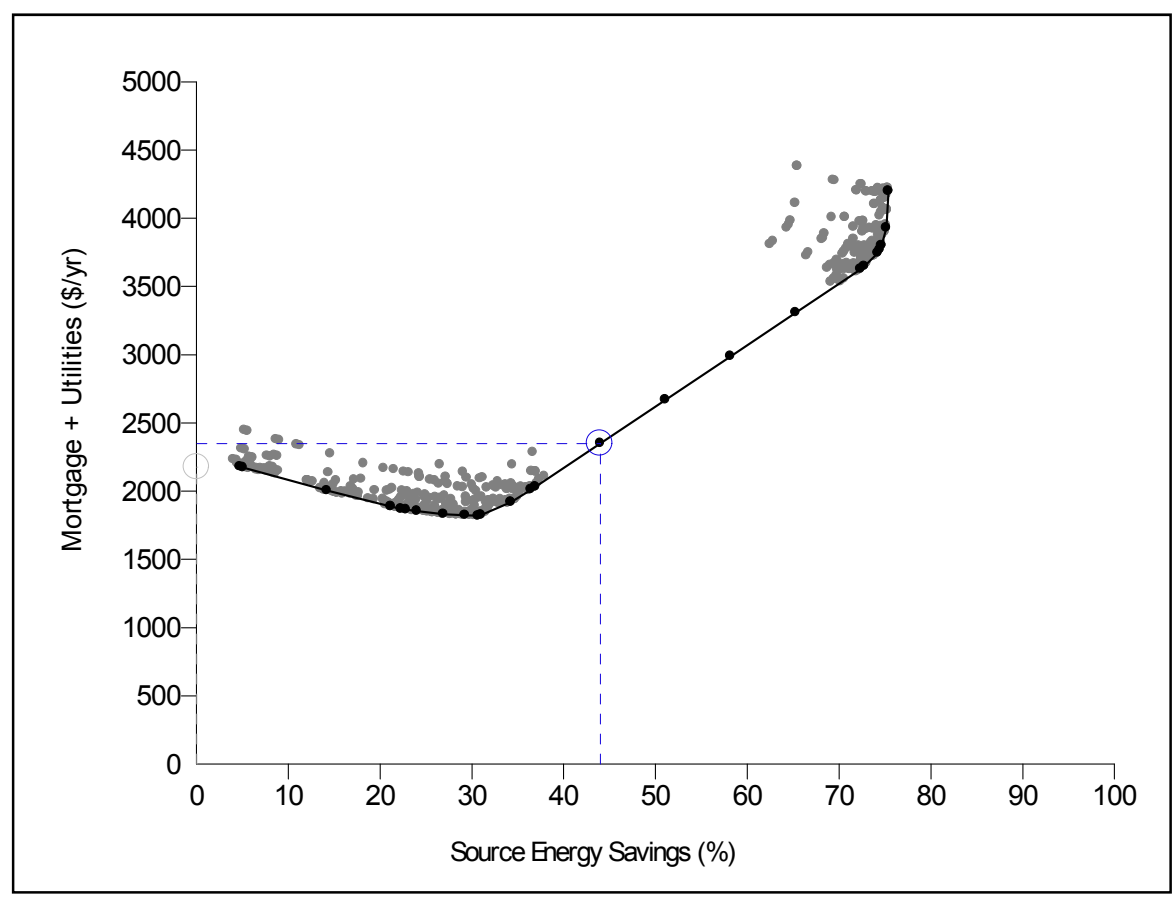

\begin{tabular}{|c|c|c|}
\hline Component & Efficiency Level & Incremental Cost \\
\hline Wall Insulation & R15 & $\$ 87$ \\
\hline Ceiling Insulation & R30 & $\$ 0$ \\
\hline Radiant Barrier & Radiant Barrier & $\$ 483$ \\
\hline Infiltration & 0.00015 SLA & $\$ 3,642$ \\
\hline Window Type & Low-e low SHGC arg & $\$ 0$ \\
\hline Slab & Uninsulated & $\$ 0$ \\
\hline Basement & No Basement & $\$ 0$ \\
\hline Crawl Space & No Crawl Space & $\$ 0$ \\
\hline Hardwired Lighting & $100 \%$ Fluorescent & $\$ 194$ \\
\hline Air Conditioner & SEER 18 & $\$ 900$ \\
\hline Furnace & AFUE $92.5 \%$ & $\$ 348$ \\
\hline Heat Pump & No Heat Pump & $\$ 0$ \\
\hline Water Heater & Gas Tankless & $\$ 625$ \\
\hline Ducts & Inside & $\$ 947$ \\
\hline Solar DHW & $32 \mathrm{sq} \mathrm{ft} \mathrm{ICS}$ & $\$ 2,654$ \\
\hline PV Size & $1.0 \mathrm{~kW}$ & $\$ 7,500$ \\
\hline Cooling Capacity Reduction & 2.5 tons & $(\$ 1,362)$ \\
\hline Heating Capacity Reduction & $-30 \mathrm{kBtu} / \mathrm{hr}$ & $\$ 103$ \\
\hline Total & & $\$ 16,121$ \\
\hline
\end{tabular}

\begin{tabular}{|l|r|}
\hline Savings on annual utility bill & $\$ 875$ \\
\hline Increase in annual mortgage payment & $\$ 1,049$ \\
\hline Net annual savings & $(\$ 174)$ \\
\hline
\end{tabular}

Figure D-10. Near-neutral-cost point for Houston with gas space- and water-heating. 


\section{Minimum-Cost / Houston / Electric Heat}

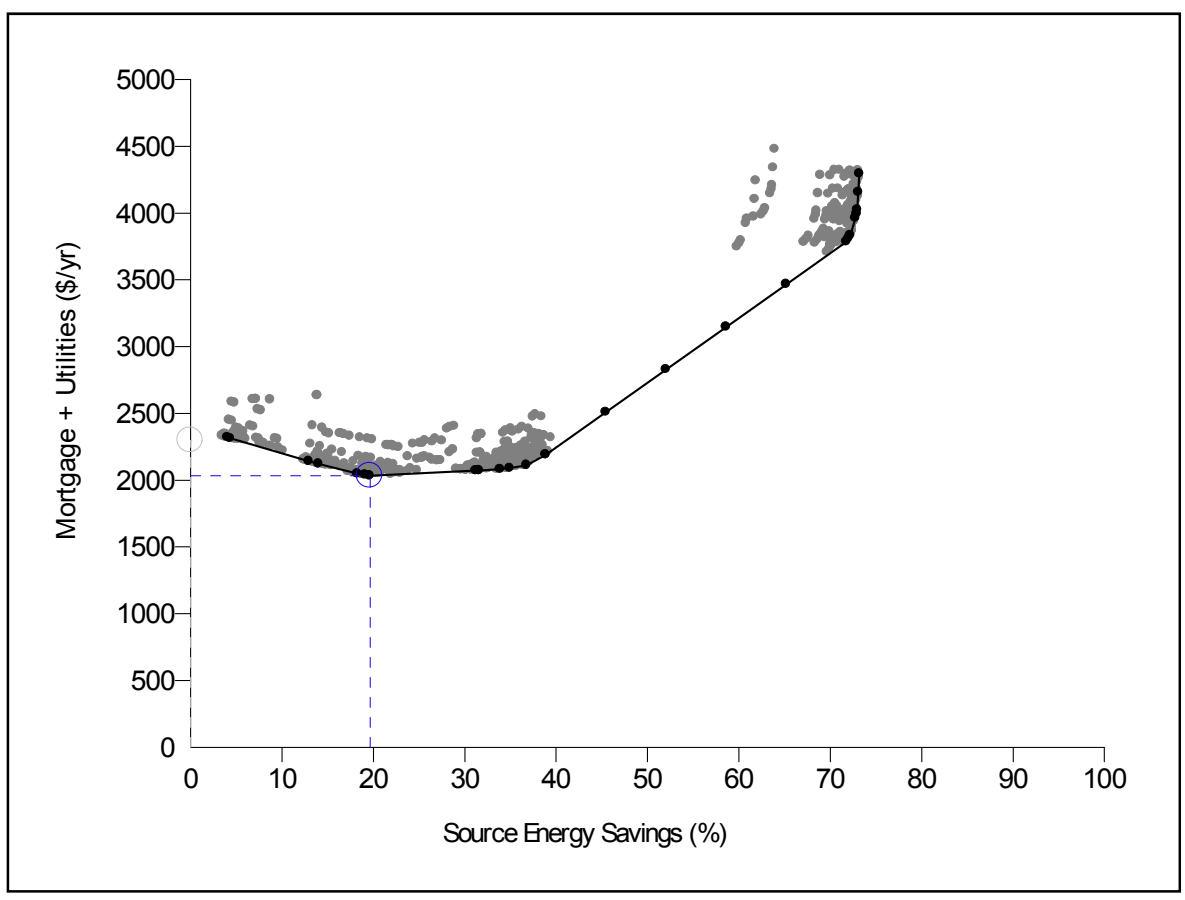

\begin{tabular}{|c|c|c|c|}
\hline Component & Efficiency Level & $\underline{\text { Incr }}$ & ntal Cost \\
\hline Wall Insulation & R15 & & $\$ 87$ \\
\hline Ceiling Insulation & R30 & & $\$ 0$ \\
\hline Radiant Barrier & None & & $\$ 0$ \\
\hline Infiltration & 0.0003 SLA & & $\$ 1,821$ \\
\hline Window Type & Low-e low SHGC arg & & $\$ 0$ \\
\hline Slab & Uninsulated & & $\$ 0$ \\
\hline Basement & No Basement & & $\$ 0$ \\
\hline Crawl Space & No Crawl Space & & $\$ 0$ \\
\hline Hardwired Lighting & $100 \%$ Fluorescent & & $\$ 194$ \\
\hline Air Conditioner & No Air Conditioner & & $\$ 0$ \\
\hline Furnace & No Furnace & & $\$ 0$ \\
\hline Heat Pump & SEER 13. HSPF 8.1 & & $\$ 0$ \\
\hline Water Heater & Electric Premium & & $\$ 114$ \\
\hline Ducts & Inside & & $\$ 947$ \\
\hline Solar DHW & No Solar DHW & & $\$ 0$ \\
\hline PV Size & $0 \mathrm{~kW}$ & & $\$ 0$ \\
\hline Cooling Capacity Reduction & 2.5 tons & & $(\$ 1,152)$ \\
\hline Heating Capacity Reduction & $-30 \mathrm{kBtu} / \mathrm{hr}$ & & $\$ 0$ \\
\hline Total & & & $\$ 2,011$ \\
\hline Savings on ann & utility bill & $\$ 412$ & \\
\hline Increase in ann & mortgage payment & $\$ 146$ & \\
\hline Net annual savi & & $\$ 266$ & \\
\hline
\end{tabular}

Figure D-11. Minimum-cost point for Houston with electric space- and water-heating. 


\section{Neutral-Cost / Houston / Electric Heat}

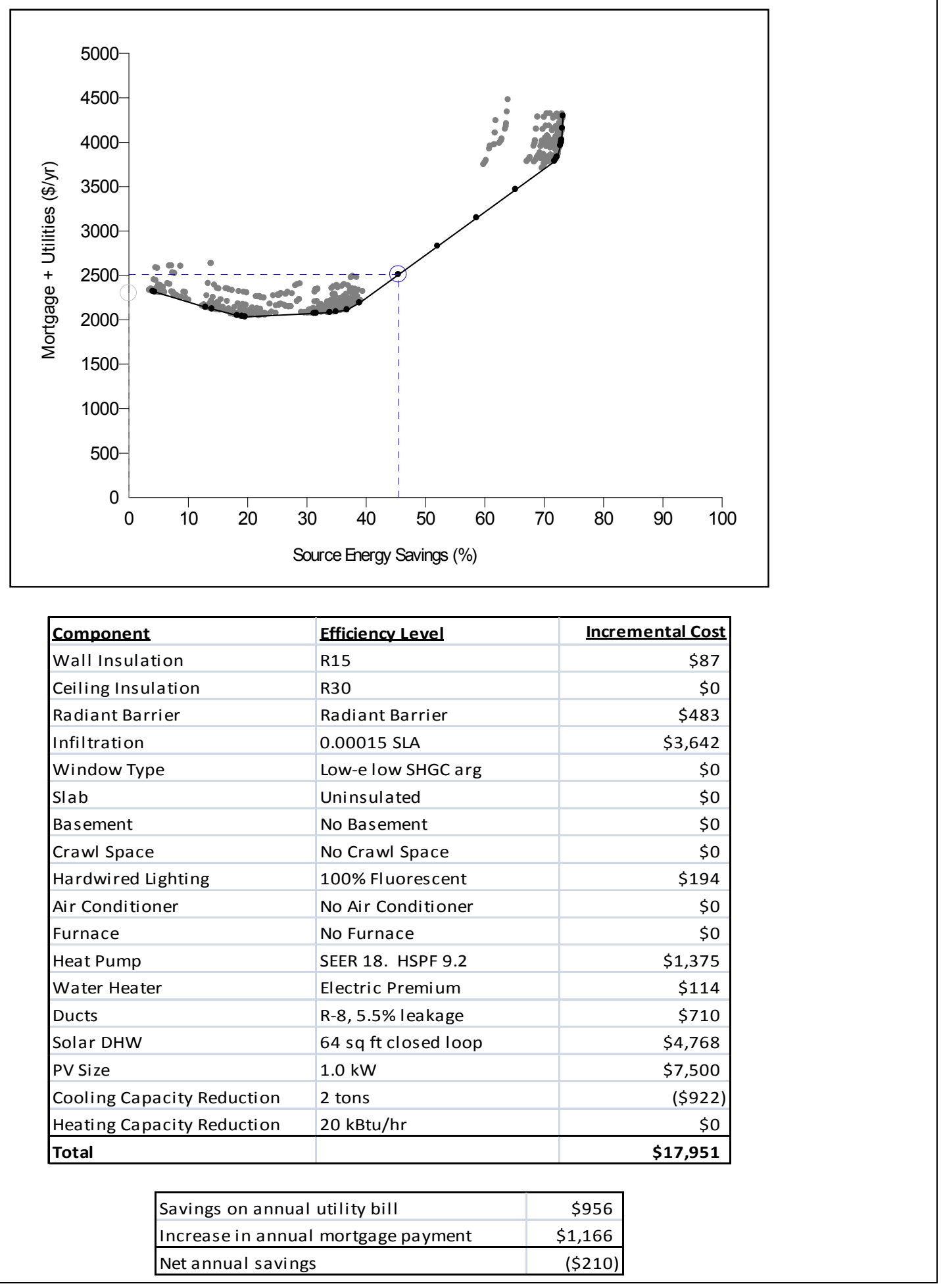

Figure D-12. Near neutral-cost point for Houston with electric space- and water-heating. 


\section{Minimum-Cost / Phoenix / Gas Heat}

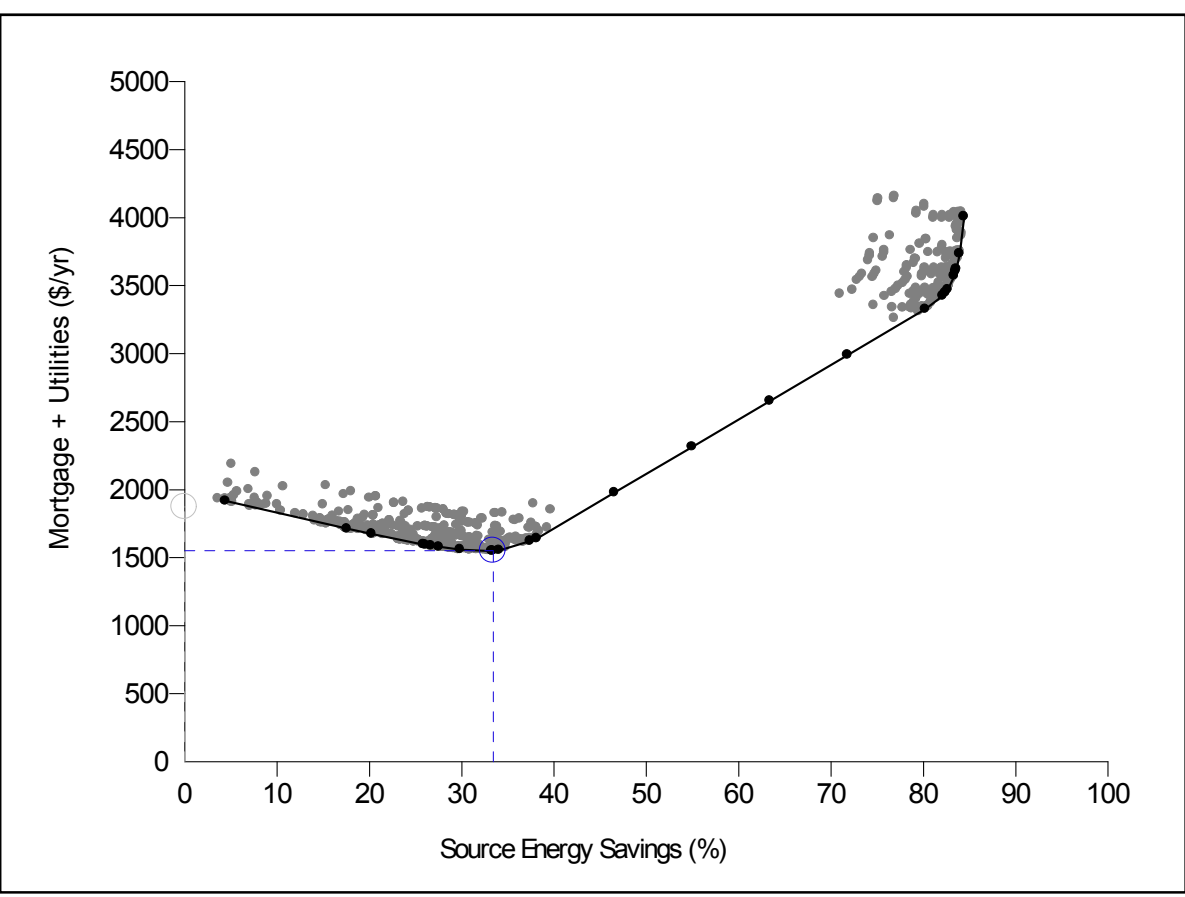

\begin{tabular}{|c|c|c|c|}
\hline Component & Efficiency Level & $\underline{\text { Incr }}$ & ntal Cost \\
\hline Wall Insulation & R15 & & $\$ 87$ \\
\hline Ceiling Insulation & R30 & & $\$ 0$ \\
\hline Radiant Barrier & None & & $\$ 0$ \\
\hline Infiltration & 0.0003 SLA & & $\$ 1,821$ \\
\hline Window Type & Low-e low SHGC arg & & $\$ 0$ \\
\hline Slab & Uninsulated & & $\$ 0$ \\
\hline Basement & No Basement & & $\$ 0$ \\
\hline Crawl Space & No Crawl Space & & $\$ 0$ \\
\hline Hardwired Lighting & 100\% Fluorescent & & $\$ 194$ \\
\hline Air Conditioner & SEER 17 & & $\$ 720$ \\
\hline Furnace & AFUE $80 \%$ & & $\$ 0$ \\
\hline Heat Pump & No Heat Pump & & $\$ 0$ \\
\hline Water Heater & Gas Tankless & & $\$ 625$ \\
\hline Ducts & Inside & & $\$ 947$ \\
\hline Solar DHW & No Solar DHW & & $\$ 0$ \\
\hline PV Size & $0 \mathrm{~kW}$ & & $\$ 0$ \\
\hline Cooling Capacity Reduction & 2 tons & & $(\$ 1,089)$ \\
\hline Heating Capacity Reduction & $30 \mathrm{kBtu} / \mathrm{hr}$ & & $(\$ 104)$ \\
\hline Total & & & $\$ 3,201$ \\
\hline Savings on ann & utility bill & $\$ 550$ & \\
\hline Increase in ann & mortgage payment & $\$ 228$ & \\
\hline Net annual savi & & $\$ 322$ & \\
\hline
\end{tabular}

Figure D-13. Minimum-cost point for Phoenix with gas space- and water-heating. 


\section{Neutral-Cost / Phoenix / Gas Heat}

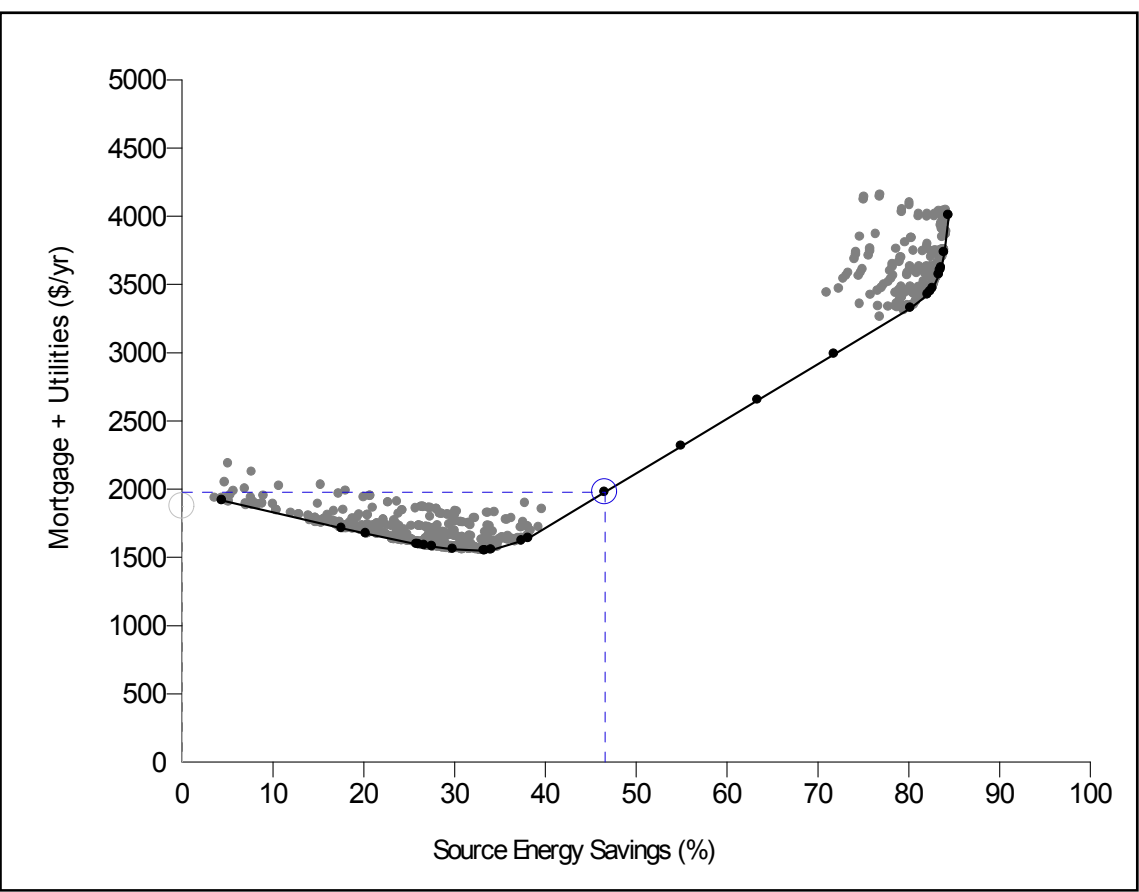

\begin{tabular}{|l|l|r|}
\hline Component & Efficiency Level & Incremental Cost \\
\hline Wall Insulation & R15 & $\$ 87$ \\
\hline Ceiling Insulation & R30 & $\$ 0$ \\
\hline Radiant Barrier & Radiant Barrier & $\$ 483$ \\
\hline Infiltration & 0.0003 SLA & $\$ 1,821$ \\
\hline Window Type & Low-e low SHGC arg & $\$ 0$ \\
\hline Slab & Uninsulated & $\$ 0$ \\
\hline Basement & No Basement & $\$ 0$ \\
\hline Crawl Space & No Crawl Space & $\$ 0$ \\
\hline Hardwired Lighting & $100 \%$ Fluorescent & $\$ 194$ \\
\hline Air Conditioner & SEER 17 & $\$ 20$ \\
\hline Furnace & AFUE 92.5\% & $\$ 348$ \\
\hline Heat Pump & No Heat Pump & $\$ 0$ \\
\hline Water Heater & Gas Tankless & $\$ 625$ \\
\hline Ducts & Inside & $\$ 947$ \\
\hline Solar DHW & 32 sq ft ICS & $\$ 2,654$ \\
\hline PV Size & 1.0 kW & $\$ 7,500$ \\
\hline Cooling Capacity Reduction & 2 tons & $(\$ 1,089)$ \\
\hline Heating Capacity Reduction & 30 kBtu/hr & \\
\hline Total & & $\$ 104)$ \\
\hline
\end{tabular}

Figure D-14. Near-neutral-cost point for Phoenix with gas space- and water-heating. 


\section{Minimum-Cost / Phoenix / Electric Heat}

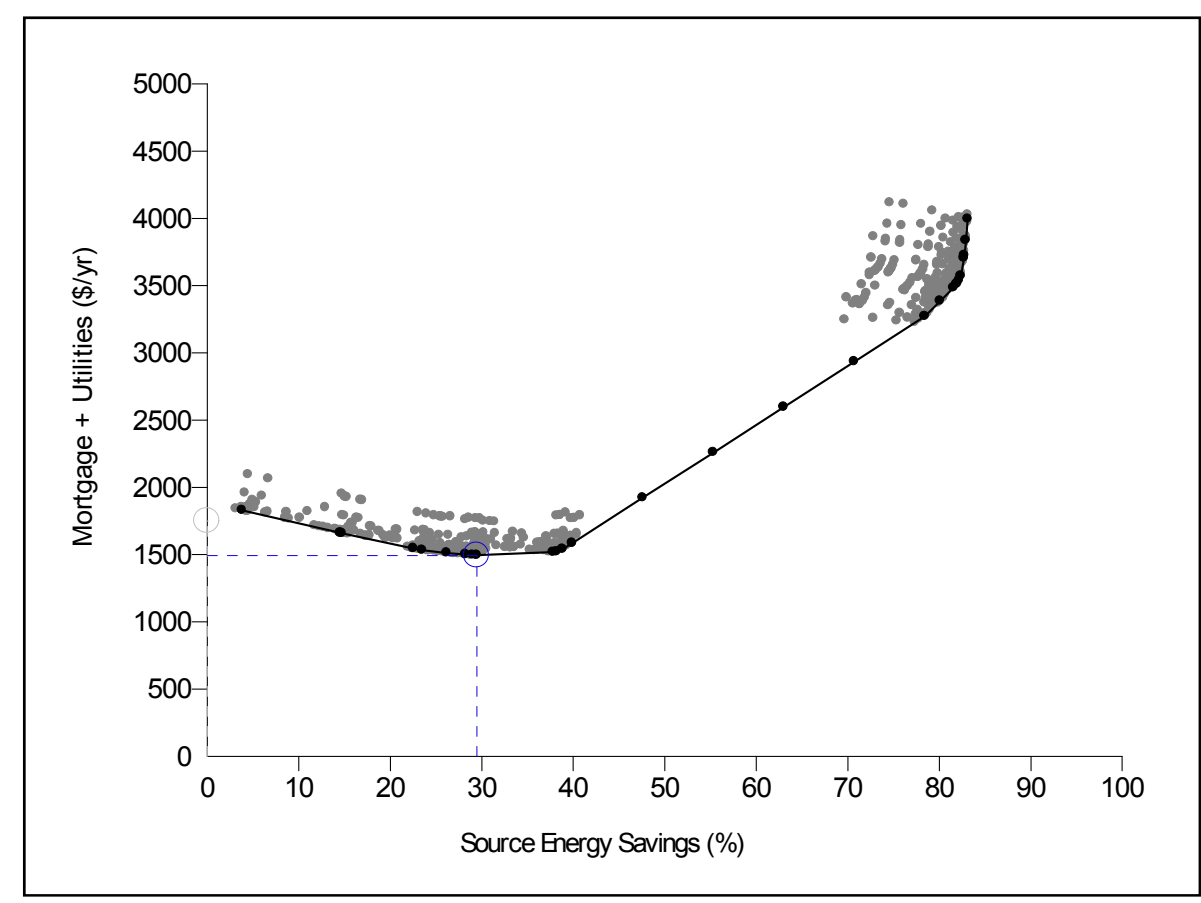

\begin{tabular}{|c|c|c|c|}
\hline Component & Efficiency Level & $\underline{\text { Incr }}$ & ntal Cost \\
\hline Wall Insulation & R15 & & $\$ 87$ \\
\hline Ceiling Insulation & R30 & & $\$ 0$ \\
\hline Radiant Barrier & None & & $\$ 0$ \\
\hline Infiltration & 0.0003 SLA & & $\$ 1,821$ \\
\hline Window Type & Low-e low SHGC arg & & $\$ 0$ \\
\hline Slab & Uninsulated & & $\$ 0$ \\
\hline Basement & No Basement & & $\$ 0$ \\
\hline Crawl Space & No Crawl Space & & $\$ 0$ \\
\hline Hardwired Lighting & $100 \%$ Fluorescent & & $\$ 194$ \\
\hline Air Conditioner & No Air Conditioner & & $\$ 0$ \\
\hline Furnace & No Furnace & & $\$ 0$ \\
\hline Heat Pump & SEER 15. HSPF 8.8 & & $\$ 550$ \\
\hline Water Heater & Electric Premium & & $\$ 114$ \\
\hline Ducts & Inside & & $\$ 947$ \\
\hline Solar DHW & No Solar DHW & & $\$ 0$ \\
\hline PV Size & $0 \mathrm{~kW}$ & & $\$ 0$ \\
\hline Cooling Capacity Reduction & 2 tons & & $(\$ 922)$ \\
\hline Heating Capacity Reduction & $30 \mathrm{kBtu} / \mathrm{hr}$ & & $\$ 0$ \\
\hline Total & & & $\$ 2,791$ \\
\hline Savings on ann & utility bill & $\$ 458$ & \\
\hline Increase in ann & mortgage payment & $\$ 204$ & \\
\hline Net annual savi & & $\$ 254$ & \\
\hline
\end{tabular}

Figure D-15. Minimum-cost point for Phoenix with electric space- and water-heating. 


\section{Neutral-Cost / Phoenix / Electric Heat}

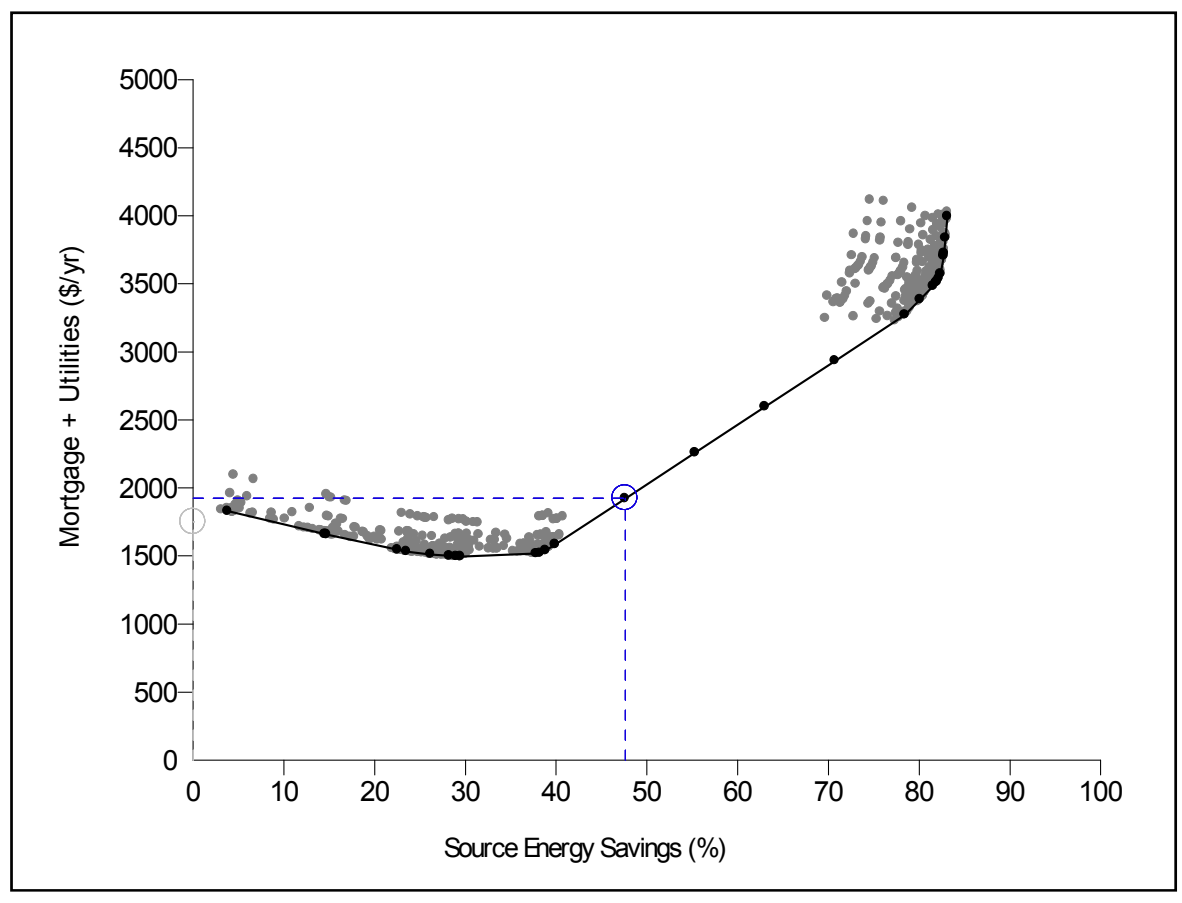

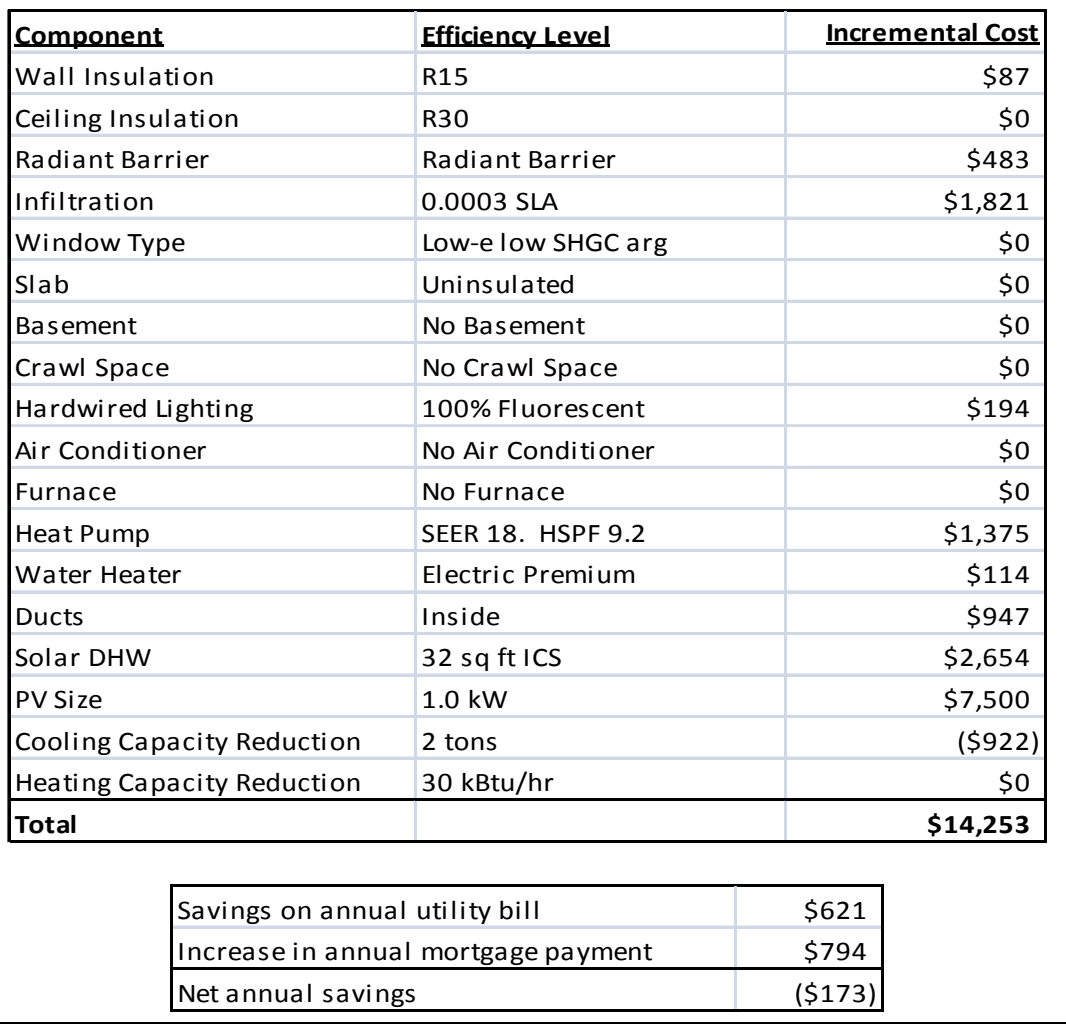

Figure D-16. Near-neutral-cost point for Phoenix with electric space- and water-heating. 


\section{Minimum-Cost / Seattle / Gas Heat}

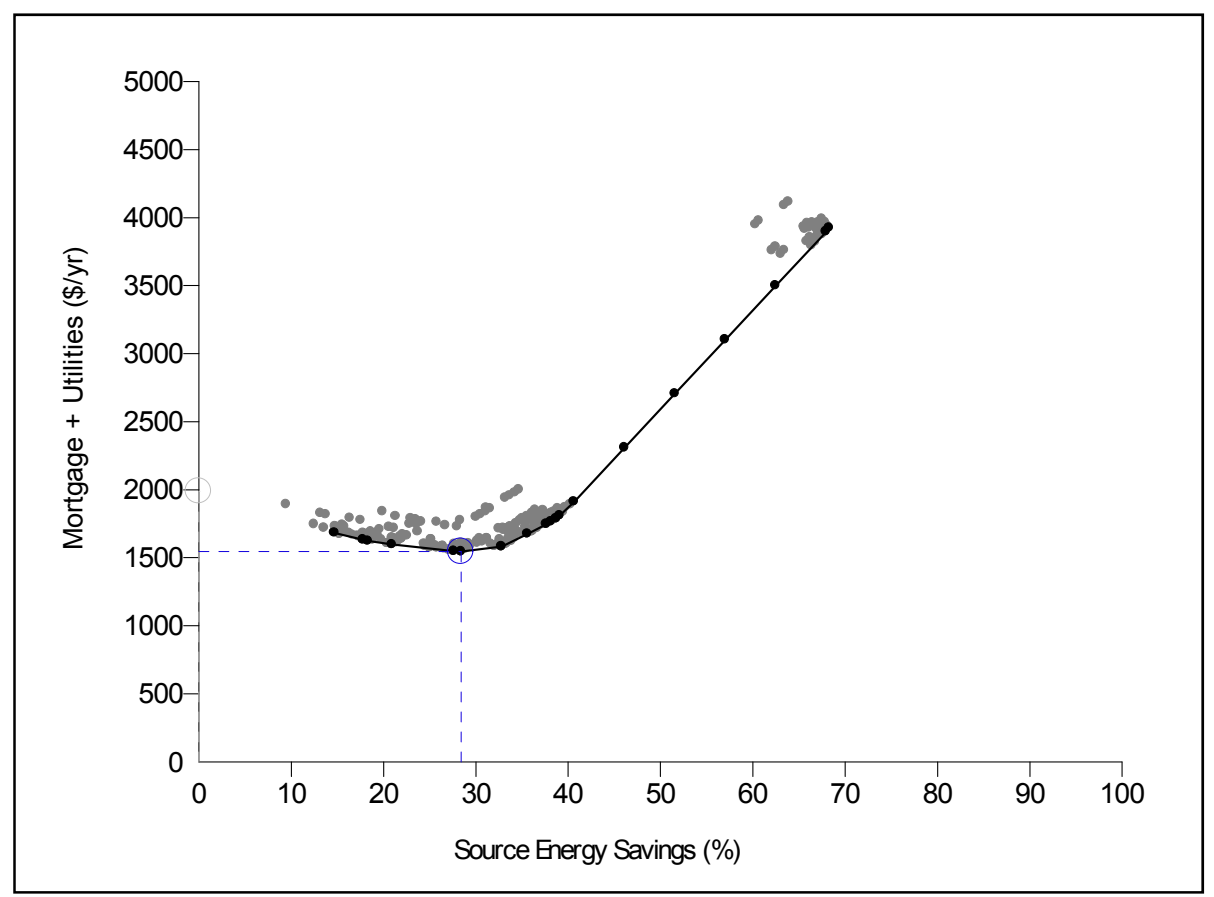

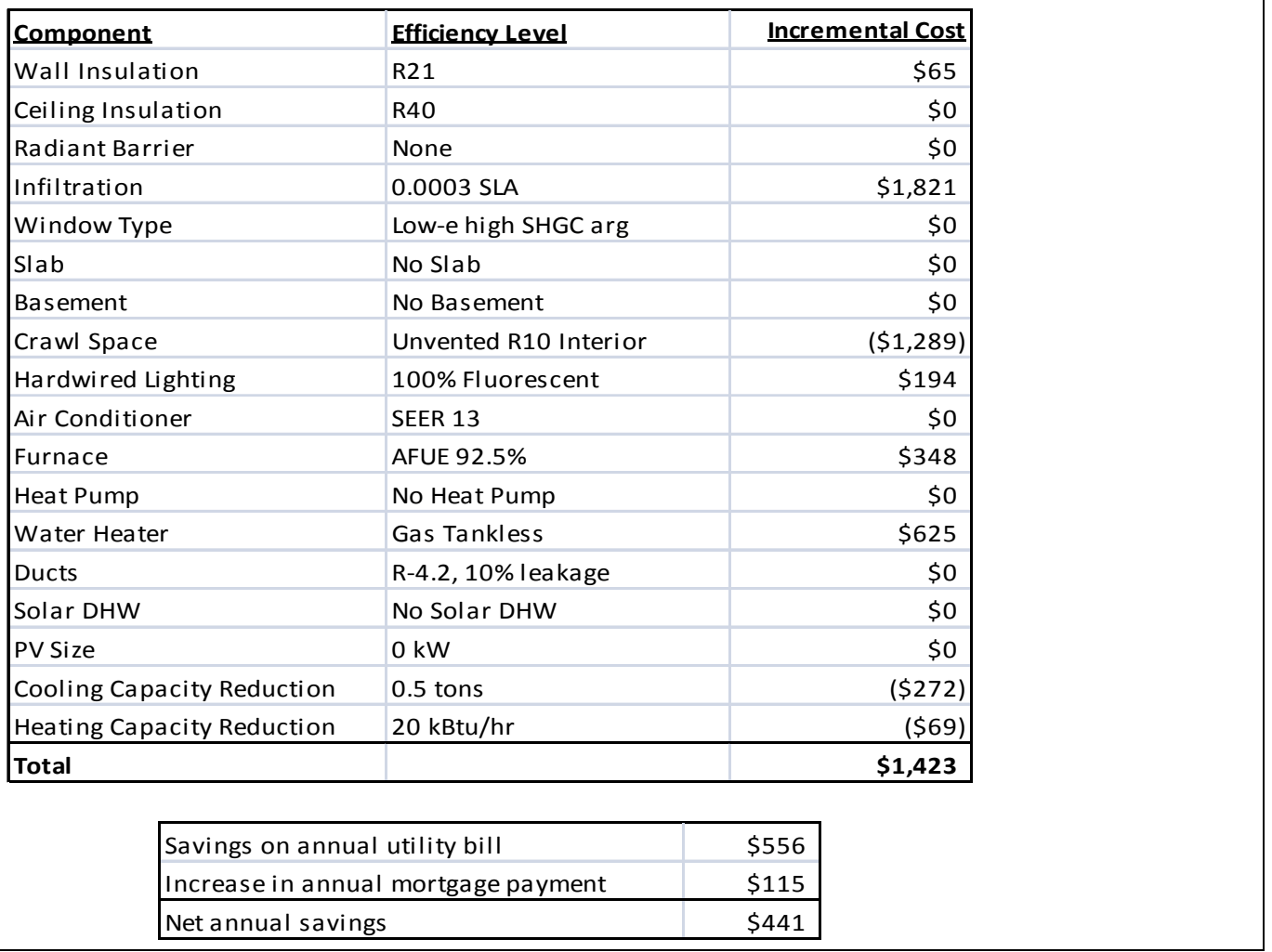

Figure D-17. Minimum-cost point for Seattle with gas space- and water-heating. 


\section{Neutral-Cost / Seattle / Gas Heat}

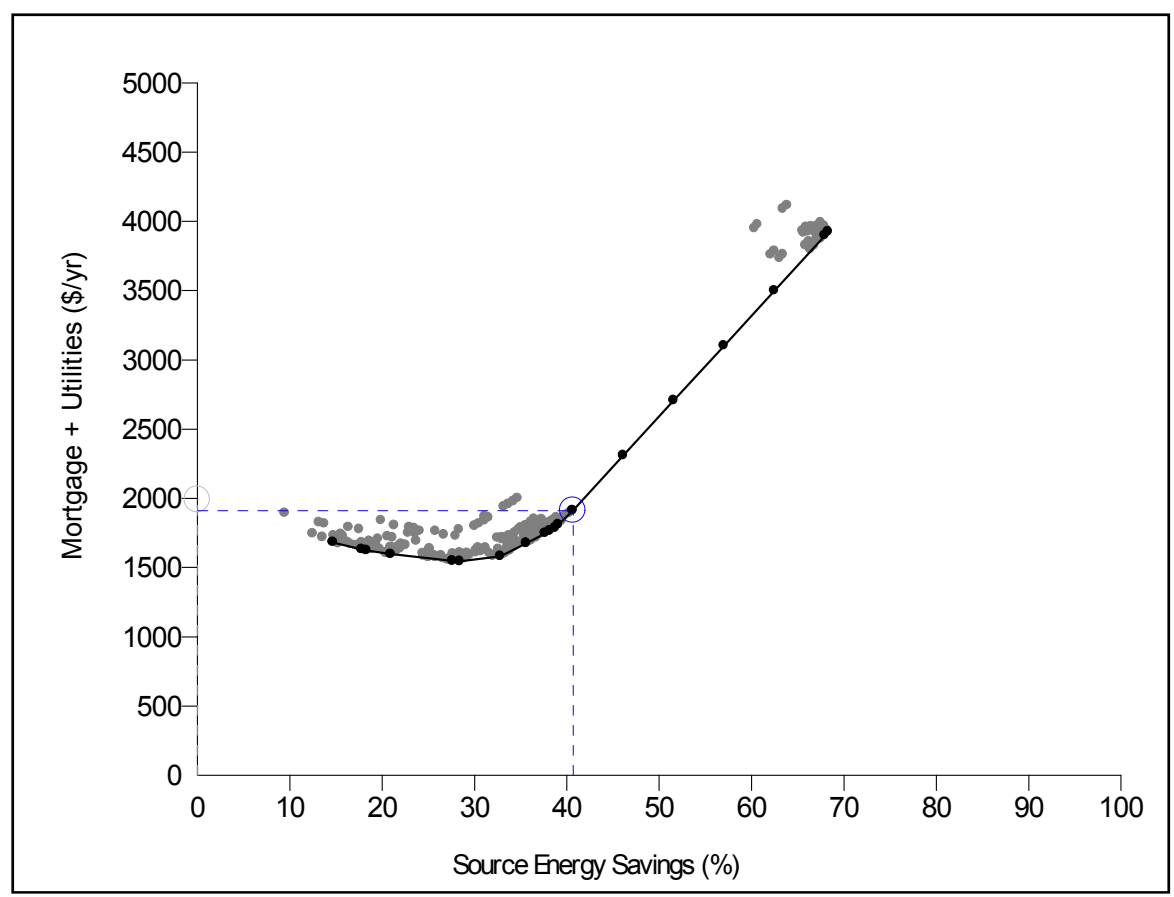

\begin{tabular}{|c|c|c|c|}
\hline Component & Efficiency Level & $\underline{\text { Incr }}$ & ntal Cost \\
\hline Wall Insulation & R21 & & $\$ 65$ \\
\hline Ceiling Insulation & R50 & & $\$ 459$ \\
\hline Radiant Barrier & None & & $\$ 0$ \\
\hline Infiltration & 0.00008 SLA & & $\$ 5,463$ \\
\hline Window Type & Low-e high SHGC arg & & $\$ 0$ \\
\hline Slab & No Slab & & $\$ 0$ \\
\hline Basement & No Basement & & \$o \\
\hline Crawl Space & Unvented R10 Interior & & $(\$ 1,289)$ \\
\hline Hardwired Lighting & $100 \%$ Fluorescent & & $\$ 194$ \\
\hline Air Conditioner & SEER 17 & & $\$ 720$ \\
\hline Furnace & AFUE $92.5 \%$ & & $\$ 348$ \\
\hline Heat Pump & No Heat Pump & & $\$ 0$ \\
\hline Water Heater & Gas Tankless & & $\$ 625$ \\
\hline Ducts & $\mathrm{R}-4.2,10 \%$ leakage & & $\$ 0$ \\
\hline Solar DHW & 64 sq ft closed loop & & $\$ 4,768$ \\
\hline PV Size & $0 \mathrm{~kW}$ & & $\$ 0$ \\
\hline Cooling Capacity Reduction & 0.5 tons & & $(\$ 272)$ \\
\hline Heating Capacity Reduction & $20 \mathrm{kBtu} / \mathrm{hr}$ & & $(\$ 69)$ \\
\hline Total & & & $\$ 11,012$ \\
\hline Savings on ann & utility bill & $\$ 839$ & \\
\hline Increase in ann & mortgage payment & $\$ 763$ & \\
\hline Net annual savi & & $\$ 76$ & \\
\hline
\end{tabular}

Figure D-18. Near-neutral-cost point for Seattle with gas space- and water-heating. 
Minimum-Cost / Seattle / Electric Heat

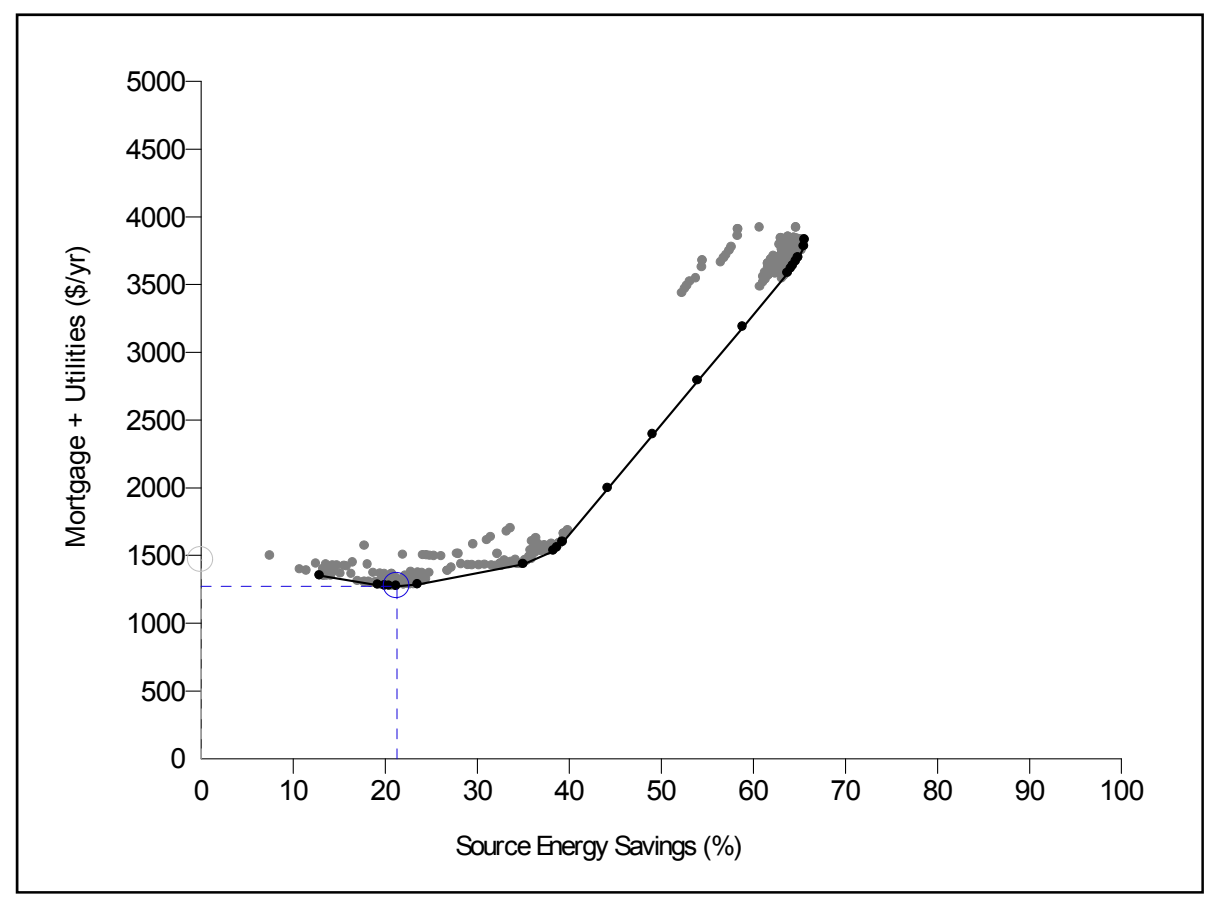

\begin{tabular}{|l|l|r|}
\hline Component & Efficiency Level & Incremental Cost \\
\hline Wall Insulation & R21 & $\$ 65$ \\
\hline Ceiling Insulation & R40 & $\$ 0$ \\
\hline Radiant Barrier & None & $\$ 0$ \\
\hline Infiltration & 0.0003 SLA & $\$ 1,821$ \\
\hline Window Type & Low-e high SHGC arg & $\$ 0$ \\
\hline Slab & No Slab & $\$ 0$ \\
\hline Basement & No Basement & $\$ 0$ \\
\hline Crawl Space & Unvented R10 Interior & $\$ 1,289)$ \\
\hline Hardwired Lighting & $100 \%$ Fluorescent & $\$ 194$ \\
\hline Air Conditioner & No Air Conditioner & $\$ 0$ \\
\hline Furnace & No Furnace & $\$ 0$ \\
\hline Heat Pump & SEER 13. HSPF 8.1 & $\$ 0$ \\
\hline Water Heater & Electric Premium & $\$ 114$ \\
\hline Ducts & R-4.2, 10\% leakage & $\$ 0$ \\
\hline Solar DHW & No Solar DHW & $\$ 0$ \\
\hline PV Size & 0 kW & $\$ 0$ \\
\hline Cooling Capacity Reduction & 0.5 tons & $\$ \$ 230)$ \\
\hline Heating Capacity Reduction & 20 kBtu/hr & $\$ 0$ \\
\hline Total & & $\$ 5$ \\
\hline
\end{tabular}

\begin{tabular}{|l|r|}
\hline Savings on annual utility bill & $\$ 262$ \\
\hline Increase in annual mortgage payment & $\$ 69$ \\
\hline Net annual savings & $\$ 193$ \\
\hline
\end{tabular}

Figure D-19. Minimum-cost point for Seattle with electric space- and water-heating. 


\section{Neutral-Cost / Seattle / Electric Heat}

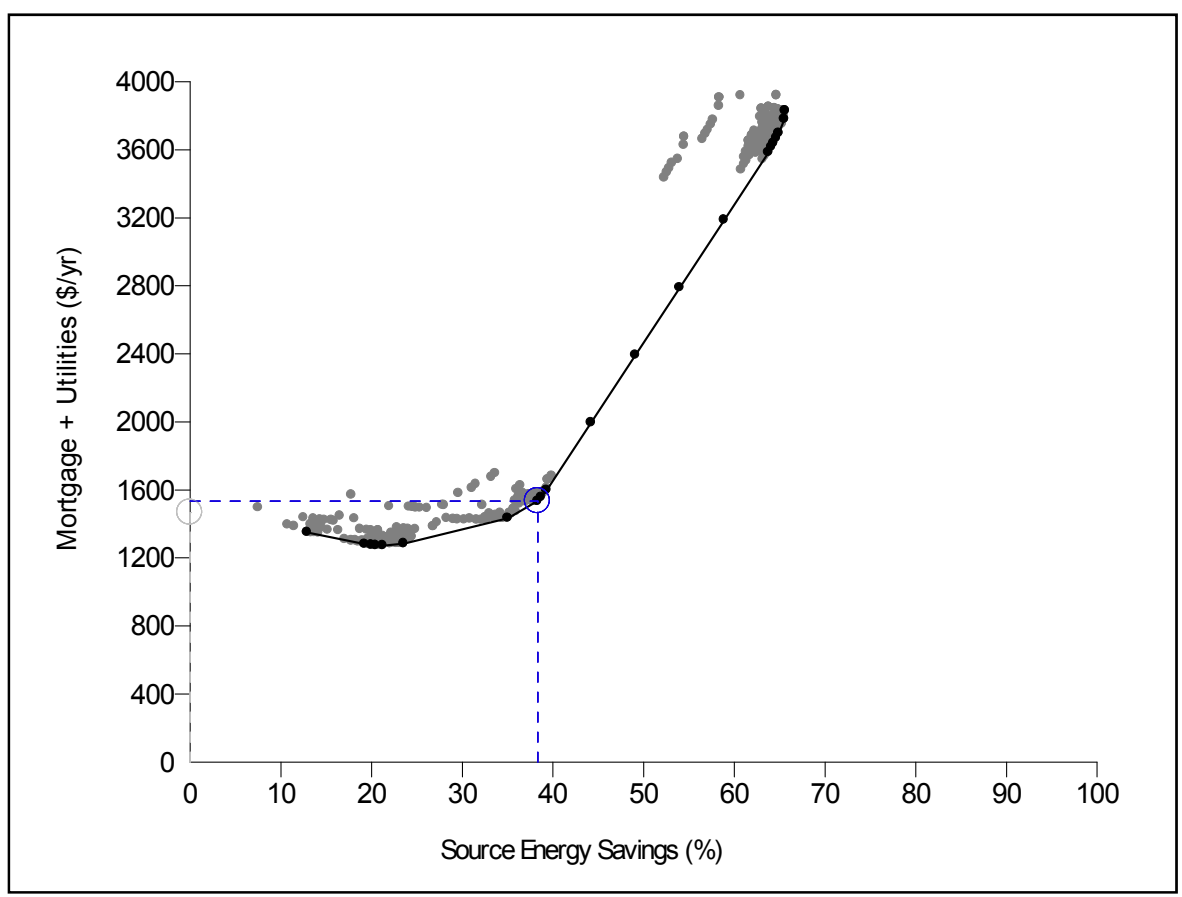

\begin{tabular}{|c|c|c|c|}
\hline \multirow{2}{*}{$\begin{array}{l}\text { Component } \\
\text { Wall Insulation }\end{array}$} & \multirow{2}{*}{$\begin{array}{l}\text { Efficiency Level } \\
\text { R21 }\end{array}$} & \multicolumn{2}{|c|}{ Incremental Cost } \\
\hline & & $\$$ & 65 \\
\hline Ceiling Insulation & R40 & $\$$ & - \\
\hline Radiant Barrier & None & $\$$ & - \\
\hline Infiltration & 0.0003 SLA & $\$$ & 1,821 \\
\hline Window Type & Low-e high SHGC arg & $\$$ & - \\
\hline Slab & No Slab & $\$$ & - \\
\hline Basement & No Basement & $\$$ & - \\
\hline Crawl Space & Unvented R10 Interior & $\$$ & $(1,289)$ \\
\hline Hardwired Lighting & $100 \%$ Fluorescent & $\$$ & 194 \\
\hline Air Conditioner & No Air Conditioner & $\$$ & - \\
\hline Furnace & No Furnace & $\$$ & - \\
\hline Heat Pump & SEER 15. HSPF 8.8 & $\$$ & 550 \\
\hline Water Heater & Electric Premium & $\$$ & 114 \\
\hline Ducts & $\mathrm{R}-4.2,10 \%$ leakage & $\$$ & - \\
\hline Solar DHW & 64 sq ft closed loop & $\$$ & 4,768 \\
\hline PV Size & $0 \mathrm{~kW}$ & $\$$ & - \\
\hline Cooling Capacity Reduction & 0.5 tons & $\$$ & $(230)$ \\
\hline Heating Capacity Reduction & $20 \mathrm{kBtu} / \mathrm{hr}$ & $\$$ & - \\
\hline Total & & $\mathbf{\$}$ & 5,993 \\
\hline \multicolumn{2}{|c|}{ Savings on annual utility bill } & $\$ 472$ & \\
\hline \multicolumn{2}{|c|}{ Increase in annual mortgage payment } & $\$ 539$ & \\
\hline \multicolumn{2}{|c|}{ Net annual savings } & $(\$ 67)$ & \\
\hline
\end{tabular}

Figure D-20. Near-neutral-cost point for Seattle with electric space- and water-heating. 


\section{REPORT DOCUMENTATION PAGE}

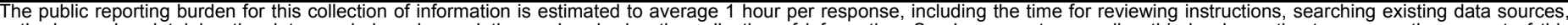

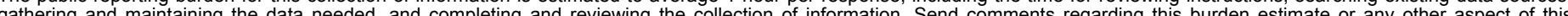

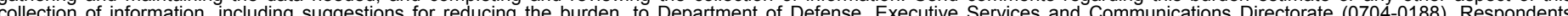

should be awa that notwithstanding any other provision of law, no person shall be subject to any penalty for falli

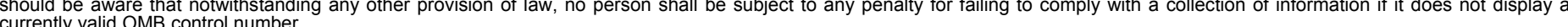

PLEASE DO NOT RETURN YOUR FORM TO THE ABOVE ORGANIZATION.
1. REPORT DATE (DD-MM-YYYY) August 2009
4. TITLE AND SUBTITLE
Technical Approach for the Development of DOE Building America
Builders Challenge Technology Information Packages (Revised)

6. AUTHOR(S)

D.R. Roberts and R. Anderson
3. DATES COVERED (From - To)

5a. CONTRACT NUMBER

DE-AC36-08-GO28308

5b. GRANT NUMBER

5c. PROGRAM ELEMENT NUMBER

5d. PROJECT NUMBER

NREL/TP-550-44687

5e. TASK NUMBER

BET98001

5f. WORK UNIT NUMBER
7. PERFORMING ORGANIZATION NAME(S) AND ADDRESS(ES)

National Renewable Energy Laboratory

1617 Cole Blvd.

Golden, CO 80401-3393

9. SPONSORING/MONITORING AGENCY NAME(S) AND ADDRESS(ES)

\section{PERFORMING ORGANIZATION REPORT NUMBER \\ NREL/TP-550-44687}

10. SPONSOR/MONITOR'S ACRONYM(S) NREL

11. SPONSORING/MONITORING AGENCY REPORT NUMBER

12. DISTRIBUTION AVAILABILITY STATEMENT

National Technical Information Service

U.S. Department of Commerce

5285 Port Royal Road

Springfield, VA 22161

13. SUPPLEMENTARY NOTES

14. ABSTRACT (Maximum 200 Words)

The U.S. Department of Energy has issued a challenge to the homebuilding industry to build 220,000 highperformance homes by 2012. The initiative is called Builders Challenge. To qualify, homes must meet the requirements of one of three compliance paths established by DOE: performance path, prescriptive path, or participating in a partner program.

15. SUBJECT TERMS

builders challenge, building america; performance path; prescriptive path

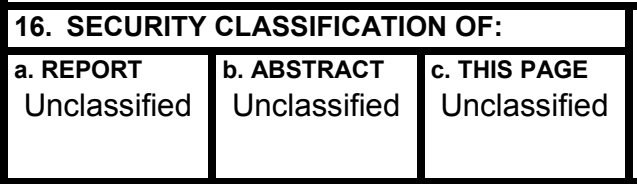

\begin{tabular}{l|l} 
17. LIMITATION & 18. \\
OF ABSTRACT & OF PAGES \\
UL &
\end{tabular}

19a. NAME OF RESPONSIBLE PERSON

19b. TELEPHONE NUMBER (Include area code) 